\title{
An investigation of the luminosity-metallicity relation for a large sample of low-metallicity emission-line galaxies ${ }^{\star \star \star \star}$
}

\author{
N. G. Guseva ${ }^{1,2}$, P. Papaderos ${ }^{3,4}$, H. T. Meyer ${ }^{5,6}$, Y. I. Izotov ${ }^{1,2}$, and K. J. Fricke ${ }^{1}$
}

\author{
1 Max-Planck-Institute for Radioastronomy, Auf dem Hügel 69, 53121 Bonn, Germany \\ e-mail: guseva@mao.kiev.ua \\ 2 Main Astronomical Observatory, Ukrainian National Academy of Sciences, Zabolotnoho 27, Kyiv 03680, Ukraine \\ 3 Instituto de Astrofísica de Andalucía (CSIC), Camino Bajo de Huétor 50, Granada 18008, Spain \\ 4 Department of Astronomy and Space Physics, Uppsala University, Box 515, 75120 Uppsala, Sweden \\ 5 Astronomisches Rechen-Institut am Zentrum für Astronomie (ZAH), Mönchhofstr. 12-14, 69120 Heidelberg, Germany \\ ${ }^{6}$ Institute for Astrophysics, University of Göttingen, Friedrich-Hund-Platz 1, 37077 Göttingen, Germany
}

Received 2 May 2009 / Accepted 18 July 2009

\section{ABSTRACT}

\begin{abstract}
Context. We present $8.2 \mathrm{~m}$ VLT spectroscopic observations of $28 \mathrm{H}$ II regions in 16 emission-line galaxies and $3.6 \mathrm{~m}$ ESO telescope spectroscopic observations of $38 \mathrm{H}$ II regions in 28 emission-line galaxies. These emission-line galaxies were selected mainly from the data release 6 (DR6) of the Sloan digital sky survey (SDSS) as metal-deficient galaxy candidates.

Aims. We collect photometric and high-quality spectroscopic data for a large uniform sample of star forming galaxies including new observations. Our aim is to study the luminosity-metallicity $(L-Z)$ relation for nearby galaxies, especially at its low-metallicity end and compare it with that for higher-redshift galaxies.

Methods. Physical conditions and element abundances in the new sample are derived with the $T_{\mathrm{e}}$-method, excluding six $\mathrm{H}$ II regions from the VLT observations and nearly two third of the $\mathrm{H}$ II regions from the $3.6 \mathrm{~m}$ observations. Element abundances for the latter galaxies were derived with the semiempirical strong-line method.

Results. From our new observations we find that the oxygen abundance in 61 out of the $66 \mathrm{H}$ II regions of our sample ranges from $12+\log \mathrm{O} / \mathrm{H}=7.05$ to 8.22 . Our sample includes 27 new galaxies with $12+\log \mathrm{O} / \mathrm{H}<7.6$ which qualify as extremely metal-poor star-forming galaxies (XBCDs). Among them are $10 \mathrm{H}$ II regions with $12+\log \mathrm{O} / \mathrm{H}<7.3$. The new sample is combined with a further 93 low-metallicity galaxies with accurate oxygen abundance determinations from our previous studies, yielding in total a high-quality spectroscopic data set of $154 \mathrm{H}$ II regions. 9000 more galaxies with oxygen abundances, based mainly on the $T_{\mathrm{e}}$-method, are compiled from the SDSS. Photometric data for all galaxies of our combined sample are taken from the SDSS database while distances are from the NED. Our data set spans a range of 8 mag with respect to its absolute magnitude in SDSS $g\left(-12 \gtrsim M_{g} \gtrsim-20\right)$ and nearly 2 dex in its oxygen abundance $(7.0 \lessgtr 12+\log \mathrm{O} / \mathrm{H} \lesssim 8.8$ ), allowing us to probe the $L-Z$ relation in the nearby universe down to the lowest currently studied metallicity level. The $L-Z$ relation established on the basis of the present sample is consistent with previous ones obtained for emission-line galaxies.
\end{abstract}

Key words. galaxies: abundances - galaxies: starburst - galaxies: ISM - galaxies: fundamental parameters

\section{Introduction}

It was shown more than 20 years ago that low-luminosity dwarf galaxies have systematically lower metallicities compared to more luminous galaxies (Lequeux et al. 1979; Skillman et al. 1989; Richer \& McCall 1995). This dependence, initially obtained for irregular galaxies, was later confirmed for galaxies of different morphological types (e.g. Vila-Costas \& Edmunds 1992; Kobulnicky \& Zaritsky 1999; Melbourne \& Salzer 2002; Lee et al. 2004; Pilyugin et al. 2004; Lee et al. 2006).

The differences between giant and dwarf galaxies are usually attributed to different chemical evolution of galaxies with different masses (e.g. Lequeux et al. 1979; Tremonti et al. 2004; Lee et al. 2006; Ellison et al. 2008; Gavilán et al. 2009). Thus, more efficient mechanisms seem to be at work in massive galaxies converting gas into stars and/or less efficient ones ejecting

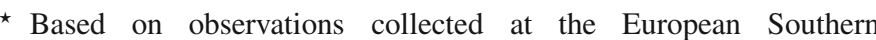
Observatory, Chile, VLT and $3.6 \mathrm{~m}$ telescopes.

$\star \star$ Tables 1-6 and Figs. 1, 2 are only available in electronic form at http: //www . aanda.org.
}

enriched matter into the galactic halo or even into the intergalactic medium. While the mass of a galaxy is one of the key physical parameters governing galaxy evolution, its determination is not easy and somewhat uncertain. Therefore, very often the luminosity, which is directly derived from observations, is used instead of the mass. In addition, some authors also use other global characteristics of a galaxy such as Hubble morphological type, rotation velocity, the gas mass fraction, surface brightness of the galaxy, to study correlations between metallicity and macroscopic properties of a galaxy (e.g. Tremonti et al. 2004; Pilyugin et al. 2004).

Metallicity reflects the level of the gas astration in the galaxy. Hence, the metallicity of a galaxy depends strongly on its evolutionary state, specifically, on the fraction of the gas converted into stars. The metallicity in emission-line galaxies is defined in terms of the relative abundance of oxygen to hydrogen (usually $12+\log \mathrm{O} / \mathrm{H}$ ) in the interstellar medium (ISM). Different mechanisms were considered in chemical evolution models to account for the low metallicity of dwarf galaxies, mainly 1) enriched galactic wind outflow which expells the newly 
synthesized heavy elements from the galaxy, resulting in slowing enrichment of the galaxy ISM; 2) inflow of metal-poor intergalactic gas and its mixing with the galaxy ISM which results in decreasing ISM metallicity; and 3) the burst character of star formation with a very low level of astration between the bursts. In principle, chemical evolution models could predict the slope and scatter of the mass-metallicity $M-Z$ (and luminositymetallicity $L-Z$ ) relations over a large range in mass (luminosity) and metallicity invoking the mechanisms mentioned above.

Usually, $L-Z$ relations are based on optical observations of nearby galaxies. However, it was shown in recent studies that the near infrared (NIR) range could be more promising for such studies. Saviane et al. (2008) collected abundances obtained by means of the temperature-sensitive method and NIR luminosities for a sample of dwarf irregular galaxies with $-20<M_{\mathrm{H}}<-13$, located in nearby groups of galaxies. They obtained a tight $M-Z$ relation with a low scatter of 0.11 dex around its linear fit. Salzer et al. (2005) (see also Vaduvescu et al. 2007) noted that the NIR luminosities are more fundamental than the $B$-band ones, since they are largely free of absorption effects and are more directly related to the stellar mass of the galaxy than optical luminosities. Nevertheless, this statement is correct only for galaxies with low and moderate SF activity. In emissionline galaxies with high star formation rate (SFR), such as blue compact dwarf (BCD) galaxies, the young, low mass-to-light $(M / L)$ ratio stellar component may provide up to $\sim 50 \%$ of the total $K$ band emission (Noeske et al. 2003). Additionally, in such systems the contribution of ionized gas to the total luminosity could be high (see e.g. Izotov et al. 1997; Papaderos et al. 1998, 2002), especially in the NIR range (see e.g. Vanzi et al. 2000; Smith \& Hancock 2009), and should be taken into account.

Recently, studies of the $L-Z$ relation were extended to larger volumes by including moderate- and high-redshift galaxies (Kobulnicky \& Zaritsky 1999; Contini et al. 2002; Maier et al. 2004). Variations of the $L-Z$ relation with redshift can provide a means to study the galaxy evolution with look-back time (see, e.g., Kobulnicky et al. 2003). It was established in this study that the slopes and zero points of the $L-Z$ relation evolve smoothly with redshift. Its large dispersion has been attributed to galaxy evolution effects. However, these results and their comparison with those for nearby galaxies should be considered with caution. The high-redshift samples are biased by different selection criteria and metallicity calibrations as compared to the local galaxies. They consist on average of more luminous and higher metallicity galaxies. Star-forming dwarf galaxies in the relatively high-redshift (up to $z \sim 1$ ) samples are rare because of their intrinsic faintness. Moreover, due to the weakness of the [O III] $\lambda 4363$ emission line in the spectra of these galaxies, their abundance determinations are more uncertain and could lead to a large scatter in the $L-Z$ diagrams. This fact could be the reason for a larger scatter of high-redshift dwarf galaxies if the direct $T_{\mathrm{e}}$-method is used instead of the empirical $R_{23}$ one (e.g., Kakazu et al. 2007).

In summary, it is difficult to obtain reliable metallicities over a large luminosity range in a homogeneous manner, i.e. employing a unique technique (e.g. the direct $T_{\mathrm{e}}$-method), even for nearby galaxies. Therefore, different methods for abundance determination are applied for galaxies of different types. The direct method is mainly used for nearby low-metallicity galaxies, while various empirical methods are used for nearby high-metallicity galaxies and for almost all high-redshift galaxies. The variety of methods results in significant differences in the $L-Z$ relations obtained with the direct $T_{\mathrm{e}}$-method and those based on strong emission line ratio calibrations, such as $R_{23}, P$-method, N2 and
O3N2 methods. These differences were reported by many authors (e.g., Pilyugin et al. 2004; Shi et al. 2005; Hoyos et al. 2005; Kakazu et al. 2007).

Large surveys, such as the Two-Degree Field Galaxy Redshift Survey (2dFGRS) and Sloan digital sky survey (SDSS), provide rich data sets for statistically improved studies of the $L-Z$ relation. For example, Lamareille et al. (2004) using more than 6000 spectra of SF galaxies at $z<0.15$ from the 2dFGRS have obtained an $L-Z$ relation that is much steeper than that for nearby irregulars and spiral galaxies. Tremonti et al. (2004) studied the mass-metallicity $(M-Z)$ relation for $53000 \mathrm{SF}$ galaxies within $z \sim 0.2$ extracted from SDSS, using their stellar continuum and line fitting method. This method is applicable because the bulk of their emission-line galaxies show weak emission lines and strong stellar absorption features, and therefore the contribution of gaseous emission to the galaxy luminosity is low. The Tremonti et al. (2004) $M-Z$ relation is relatively steep but it flattens for massive galaxies at masses above $10^{10} M_{\odot}$. On the contrary, Melbourne \& Salzer (2002) using 519 emissionline galaxies from the KPNO International Spectroscopic Survey (KISS) found that the slope of the $L-Z$ relation for luminous galaxies is steeper than that for dwarf galaxies. Nevertheless, Pilyugin et al. (2004) have compared the $L-Z$ relation based on more than 1000 published spectra of $\mathrm{H}$ II regions in spiral galaxies to that for irregular galaxies. They found that the slope of the relation for spirals is slightly shallower than the one for irregular galaxies. Furthermore, using 72 star-forming galaxies, Shi et al. (2005) have also shown that the slope of the $L-Z$ relation for luminous galaxies is slightly shallower than that for dwarf galaxies.

Is the slope of the $L-Z$ relation invariant for galaxies of different type, such as local dwarf and spiral galaxies and high-redshift galaxies? If differences in the $L-Z$ relations for intermediate- and high-redshift and local ones are present, they may yield important constraints on the Star Formation History (SFH) of galaxies. For this, an as accurate as possible $L-Z$ relation, based on homogeneous high-quality photometric and spectroscopic data is required for galaxies that covers a large range in metallicity and luminosity. In particular, probing the slope of the $L-Z$ relation in its low-metallicity end, i.e. in the range expected for unevolved low-mass galaxies in the faraway universe, is much needed. For this purpose, in this paper we focus our study on the lowest-metallicity galaxy candidates selected from large spectroscopic surveys, using deep follow-up spectroscopic observations.

Specifically, the objective of the work is to study the $L-Z$ relation for a large uniform sample of emission-line galaxies in the local Universe for which the element abundances are obtained with high precision. The main feature of our sample is that it is one of the richest currently available at the low-metallicity end. For the galaxy selection we used different surveys such as 2dFGRS, SDSS and others. Most of our sample galaxies currently undergo strong episodes of star formation (SF).

We performed $3.6 \mathrm{~m}$ ESO spectroscopic observations of a sample of $38 \mathrm{H}$ II regions in 28 emission-line galaxies and $8.2 \mathrm{~m}$ VLT spectroscopic observations of a sample of $28 \mathrm{H} \mathrm{II} \mathrm{re-}$ gions in 16 emission-line galaxies. We supplement our new data with our previous data collected from the MMT observations (Izotov \& Thuan 2007), and from the $3.6 \mathrm{~m}$ ESO observations (Guseva et al. 2007; Papaderos et al. 2008) of the lowmetallicity emission-line galaxies selected from the SDSS, with the sample used by Izotov \& Thuan (2004) to study the helium abundance in low-metallicity BCDs (henceforth referred to as the HeBCD sample) and with the MMT sample used by 
Thuan \& Izotov (2005) to study high-ionization emission lines in low-metallicity BCDs. Our MMT, $3.6 \mathrm{~m}$ ESO and HeBCD low-metallicity galaxies were selected from different surveys (a more complete description of the MMT, $3.6 \mathrm{~m}$ ESO and HeBCD subsamples can be found in Izotov \& Thuan 2007; Guseva et al. 2007; Papaderos et al. 2008; Izotov \& Thuan 2004). During past years we selected from the SDSS and performed follow-up spectroscopic observations with large telescopes of (i) BCDs with strong ongoing SF, i.e. galaxies with high $E W(\mathrm{H} \beta)$, blue colours, high ionisation parameter; and (ii) low-metallicity galaxies in a relatively quiescent phase of $\mathrm{SF}$, i.e. galaxies with low $E W(\mathrm{H} \beta)$, low ionisation parameter or older starburst age. For this, we selected galaxies with weak or not detected [O III] $\lambda 4363$ emission line and with $[\mathrm{O}$ III $] \lambda 4959 / \mathrm{H} \beta \lesssim 1$ and $[\mathrm{N} \mathrm{II}] \lambda 6583 / \mathrm{H} \beta \lesssim 0.05$ (Izotov et al. 2006b; Izotov \& Thuan 2007).

SDSS is an excellent source of both photometric and spectroscopic data for more than one million galaxies in its data release 7 (DR7) (Abazajian et al. 2009). Despite that, our stringent selection criteria resulted in a very small sample of extremely metal-deficient emission-line galaxies with reliable abundance determinations. This sample is supplemented for the purpose of comparison by a sample of $~ 9000$ SDSS emission-line galaxies (SDSS sample) over a larger range of metallicities. The oxygen abundances for the galaxies from the SDSS sample are obtained using the direct $T_{\mathrm{e}}$-method. In addition, only high-quality spectra of SDSS galaxies with the non-detected [O III] $\lambda 4363$ emission line are included, for which oxygen abundances are derived by a semiempirical method (Izotov \& Thuan 2007).

Thus, we construct a large homogeneous sample with uniform selection criteria, uniform data reduction methods, and uniform techniques for the element abundance determinations. The apparent $g$ magnitudes for our entire data set are taken from the SDSS. They were used to derive absolute $g$ magnitudes which were corrected for the Galactic extinction and Virgo cluster infall, except for the comparison SDSS sample galaxies. For the latter galaxies the absolute magnitude was derived from the observed redshift, adopting a Hubble constant of $H_{0}=75 \mathrm{~km} \mathrm{~s}^{-1} \mathrm{Mpc}^{-1}$.

The paper is organized as follows. Observations and data reduction are described in Sect. 2. Physical conditions and element abundances in the galaxies from the new observations are presented in Sect. 3. We discuss the properties of the $L-Z$ relation in Sect. 4 and summarise our conclusions in Sect. 5.

\section{Observations and data reduction}

The new spectra of the $3.6 \mathrm{~m}$ ESO sample were obtained on 14-16 September, 2007 with the spectrograph EFOSC2. The grism Gr\#7 and a long slit with the width of 1'. 2 were used yielding a wavelength coverage of $\lambda \lambda 3400-5200 \AA$. The long slit was centered on the brightest part of each galaxy and simultaneously on another H II regions, whenever present. The name of each galaxy with its different H II regions, the coordinates RA, Dec. (J2000.0), date of observation, exposure time, number of exposures for each observation, average airmass and seeing are given in Table 1. All spectra were obtained at low airmass or with the slit oriented along the parallactic angle, so no corrections for atmospheric refraction have been applied.

The new VLT spectra were obtained during several runs in October - December, 2006 and January, 2007 with the spectrograph FORS2 mounted at the ESO VLT UT2. The observing conditions were photometric during the nights with seeing $<1^{\prime \prime}$. 5. Several observations were performed under excellent seeing conditions $\left(<0{ }^{\prime} 8\right)$. The grisms 600B $(\lambda \lambda \sim 3400-6200)$ and
600RI and filter GG435 ( $\lambda \lambda \sim 5400-8620)$ for the blue and red parts of the spectrum, respectively, were used. A $1^{\prime \prime} \times 360^{\prime \prime}$ long slit was centered on the brightest H II regions of each galaxy. In Table 2, the same parameters as in Table 1 are given for the VLT observations. Note that for each galaxy the first and the second lines are related to the observations in the blue and red ranges, respectively. Again, as for the EFOSC2 spectra, the observations were obtained at low airmass, and no corrections for atmospheric refraction were applied.

The data were reduced with the IRAF ${ }^{1}$ software package. This included bias-subtraction, flat-field correction, cosmic-ray removal, wavelength calibration, night sky background subtraction, correction for atmospheric extinction and absolute flux calibration of the two-dimensional spectrum. The spectra were also corrected for interstellar extinction using the reddening curve of Whitford (1958). One-dimensional spectra of one or several H II regions in each galaxy were extracted from twodimensional observed spectra. The flux- and redshift-calibrated one-dimensional EFOSC2 $3.6 \mathrm{~m}$ spectra of the $\mathrm{H}$ II regions are shown in Fig. 1 for all galaxies given in Table 1. Onedimensional VLT spectra are shown in Fig. 2 for 28 objects listed in Table 2. For VLT spectra of the four background galaxies and $\mathrm{H}$ II region No. 2 in the galaxy J2354-0004 without a detectable [O III] $\lambda 4363 \AA$ A emission line, no abundance determination has been done.

Emission-line fluxes were measured using Gaussian profile fitting. The errors of the line fluxes were calculated from the photon statistics in the non-flux-calibrated spectra. They have been propagated in the calculations of the elemental abundance errors. The quality of the VLT data reduction could be verified by a comparison of He I $\lambda 5876$ emission line fluxes measured in the blue and red spectra of the same object. We found that the fluxes of the He I $\lambda 5876$ emission line in spectra of bright objects differ by no more than 1-2\% indicating an accuracy in the flux calibration at the same level. For faint objects the difference between the flux of the He I $\lambda 5876$ emission line in the blue and red spectra is higher, $\sim 5-10 \%$, and is comparable to the statistical errors listed in Table 4.

The extinction coefficient $C(\mathrm{H} \beta)$ and equivalent widths of the hydrogen absorption lines $E W(\mathrm{abs})$ are calculated simultaneously, minimizing the deviations of corrected fluxes $I(\lambda) / I(\mathrm{H} \beta)$ of all hydrogen Balmer lines from their theoretical recombination values as

$$
\begin{aligned}
\frac{I(\lambda)}{I(\mathrm{H} \beta)}= & \frac{F(\lambda)}{F(\mathrm{H} \beta)} \frac{E W(\lambda)+|E W(\mathrm{abs})|}{E W(\lambda)} \frac{E W(\mathrm{H} \beta)}{E W(\mathrm{H} \beta)+|E W(\mathrm{abs})|} \\
& \times 10^{C(\mathrm{H} \beta) f(\lambda)} .
\end{aligned}
$$

Here $f(\lambda)$ is the reddening function normalized to the value at the wavelength of the $\mathrm{H} \beta$ line, $F(\lambda) / F(\mathrm{H} \beta)$ are the observed hydrogen Balmer emission line fluxes relative to that of $\mathrm{H} \beta$, $E W(\lambda)$ and $E W(\mathrm{H} \beta)$ the equivalent widths of emission lines, and $E W(\mathrm{abs})$ the equivalent widths of hydrogen absorption lines which we assumed to be the same for all hydrogen lines. For $f(\lambda)$ we adopted the reddening law by Whitford (1958). The extinction-corrected fluxes of emission lines other than hydrogen ones are derived from equation

$$
\frac{I(\lambda)}{I(\mathrm{H} \beta)}=\frac{F(\lambda)}{F(\mathrm{H} \beta)} \times 10^{C(\mathrm{H} \beta) f(\lambda)}
$$

1 IRAF is the Image Reduction and Analysis Facility distributed by
the National Optical Astronomy Observatory, which is operated by the
Association of Universities for Research in Astronomy (AURA) under cooperative agreement with the National Science Foundation (NSF). 
The extinction-corrected emission line fluxes $I(\lambda)$ relative to the $\mathrm{H} \beta$ fluxes multiplied by 100 , the extinction coefficients $C(\mathrm{H} \beta)$, the equivalent widths $E W(\mathrm{H} \beta)$, the observed $\mathrm{H} \beta$ fluxes $F(\mathrm{H} \beta)$ (in units $10^{-16} \mathrm{erg} \mathrm{s}^{-1} \mathrm{~cm}^{-2}$ ), and the equivalent widths of the hydrogen absorption lines are listed in Table 3 (3.6 m ESO observations) and in Table 4 (VLT observations). $C(\mathrm{H} \beta)$ and $E W$ (abs) are set to zero in Tables 3 and 4 if we do not have enough observational data or their values are negative.

\section{Physical conditions and element abundances}

The electron temperature $T_{\mathrm{e}}$, the ionic and total heavy element abundances were derived following Izotov et al. (2006a). In particular, for the ions $\mathrm{O}^{2+}, \mathrm{Ne}^{2+}$ and $\mathrm{Ar}^{3+}$ we adopt the temperature $T_{\mathrm{e}}(\mathrm{O}$ III $)$ directly derived from the [O III] $\lambda 4363 /(\lambda 4959+\lambda 5007)$ emission-line ratio. For $T_{\mathrm{e}}(\mathrm{O}$ II $)$ and $T_{\mathrm{e}}(\mathrm{S}$ III) we use the relation between the electron temperatures $T_{\mathrm{e}}\left(\mathrm{O}\right.$ III) and the temperatures characteristic for ions $\mathrm{O}^{+}$ and $\mathrm{S}^{2+}$ obtained by Izotov et al. (2006a) from the H II photoionization models based on recent stellar atmosphere models and improved atomic data (Stasińska \& Izotov 2003).

We use $T_{\mathrm{e}}(\mathrm{O}$ II $)$ for the calculation of $\mathrm{O}^{+}, \mathrm{N}^{+}, \mathrm{S}^{+}$and $\mathrm{Fe}^{2+}$ abundances and $T_{\mathrm{e}}\left(\mathrm{S}\right.$ III) for the calculation of $\mathrm{S}^{2+}, \mathrm{Cl}^{2+}$ and $\mathrm{Ar}^{2+}$ abundances. The electron number densities for some $\mathrm{H}$ II regions were obtained from the $[\mathrm{S}$ II] $\lambda 6717 / \lambda 6731$ emission line ratio. These lines were not observed or not measured in the remaining $\mathrm{H}$ II regions. For the abundance determination in those $\mathrm{H}$ II regions we adopt $N_{\mathrm{e}}=10 \mathrm{~cm}^{-3}$. The precise value of the electron number density makes little difference in the derived abundances since in the low-density limit which holds for the $\mathrm{H}$ II regions considered here, the element abundances do not depend sensitively on $N_{\mathrm{e}}$. The electron temperatures $T_{\mathrm{e}}(\mathrm{O}$ III $)$, $T_{\mathrm{e}}(\mathrm{O}$ II), the ionization correction factors (ICFs), the ionic and total $\mathrm{O}$ and $\mathrm{Ne}$ abundances are given in Table 5 for $3.6 \mathrm{~m}$ observations. The electron temperatures $T_{\mathrm{e}}(\mathrm{O}$ III $), T_{\mathrm{e}}(\mathrm{O}$ II $), T_{\mathrm{e}}(\mathrm{S}$ III $)$, electron number density $N_{\mathrm{e}}([\mathrm{S} \mathrm{II}])$, the ionization correction factors (ICFs), the ionic and total $\mathrm{O}, \mathrm{N}, \mathrm{Ne}, \mathrm{S}, \mathrm{Cl}, \mathrm{Ar}$ and $\mathrm{Fe}$ abundances are given in Table 6 for VLT observations.

The oxygen abundances $12+\log \mathrm{O} / \mathrm{H}$ in $61 \mathrm{H}$ II regions out of 66 obtained from the new $3.6 \mathrm{~m}$ ESO and VLT observations range from 7.05 to 8.22. Among them, $27 \mathrm{H}$ II regions with $12+\log \mathrm{O} / \mathrm{H}<7.6$ are found, including $10 \mathrm{H}$ II regions with $12+\log \mathrm{O} / \mathrm{H}<7.3$. The combined sample consisting of the new observations, $43 \mathrm{BCD}$ from the HeBCD sample, 30 galaxies from our previous $3.6 \mathrm{~m}$ ESO observations and 20 galaxies from the MMT observations yields a data set of $154 \mathrm{H}$ II regions. For comparison, we also use $\sim 9000$ SDSS emission-line galaxies with the [O III] $\lambda 4363$ emission line detected at least at the $1 \sigma$ level, allowing abundance determination by the direct $T_{\mathrm{e}}$-method. In addition, SDSS galaxies with high-quality spectra where the [O III] $\lambda 4363$ emission line was not detected are used. In the latter case, the oxygen abundances were derived by the semiempirical method. SDSS galaxies from the comparison sample mostly populate the high-metallicity, high-luminosity ranges, as compared to the galaxies from our combined sample of low-metallicity emission-line galaxies (Figs. 5-7). The considered galaxies span two dex in gas-phase oxygen abundance, from $12+\log \mathrm{O} / \mathrm{H} \sim 7.0$ through $\sim 9.0$.

We use SDSS $g$ magnitudes for the determination of the absolute magnitude $M_{g}$ of all galaxies from our samples, while usually $B$ magnitudes and $M_{B}$ are considered in the literature. However, Papaderos et al. (2008) have shown that for regions with ongoing bursts of star formation, which is the case for our sample galaxies, the $B-g$ colour index is of the order of $0.1 \mathrm{mag}$ only and $<0.3$ mag during the first few Gyr of galactic evolution. Therefore, we do not transform $M_{g}$ to $M_{B}$ and directly compare $M_{g}$ 's for the galaxies from our samples with $M_{B}$ 's for the galaxies available from the literature. The use of the SDSS g-band photometry for all our samples allows us to investigate the $L-Z$ relation over the $M_{g}$ range from -21 mag to the faintest magnitude of $\sim-12$ mag at the low-metallicity end.

\section{Results}

\subsection{Luminosity-metallicity relation}

In order to illustrate the main properties of our sample we plot (a) the reddening parameter $C(\mathrm{H} \beta)$ obtained from the Balmer decrement and (b) the $\mathrm{H} \beta$ equivalent width (Fig. 3) and the logarithm of the $\mathrm{H} \beta$ line luminosity (in $\mathrm{erg} \mathrm{s}^{-1}$ ) (Fig. 4) as a function of absolute magnitude $M_{g}$. The new $3.6 \mathrm{~m}$ telescope and VLT data are shown by open circles and stars, respectively. The metal-poor galaxies collected from previous $3.6 \mathrm{~m}$ ESO observations are shown by filled triangles (Guseva et al. 2007; Papaderos et al. 2008). Filled circles denote the data from the HeBCD sample collected by Izotov et al. (2004a) and Izotov \& Thuan (2004). The MMT data (Izotov \& Thuan 2007) are shown by large filled circles. The comparison SDSS sample is represented by asterisks. From the latter sample H II regions in nearby spiral galaxies are excluded, as are faint SDSS galaxies with $m_{g}>18$, the nearest SDSS galaxies with the redshift $z<0.004$ and all SDSS galaxies with $\sigma[I(4363)] / I(4363)>0.25$, totaling 443 SDSS galaxies from the comparison sample.

Our sample does not show any trend with absolute magnitude of either $C(\mathrm{H} \beta)$ or $E W(\mathrm{H} \beta)$, contrary to what was obtained by Salzer et al. (2005) for the KISS sample. The extinction in our sample galaxies is low. Only a few galaxies have $C(\mathrm{H} \beta)>0.4$. The range of $E W(\mathrm{H} \beta) \sim 0-300 \AA$ for the galaxies from our sample is similar to that for the KISS sample (Salzer et al. 2005) but it is higher than that for the high-redshift galaxies of Kobulnicky et al. (2003) where $E W(\mathrm{H} \beta) \leq 60 \AA$.

The logarithm of the $\mathrm{H} \beta$ luminosity $\log L(\mathrm{H} \beta)$ of our galaxies ranges from 36 to 42 (Fig. 4). For comparison, the galaxies from the KISS sample by Salzer et al. (2005) and intermediate-redshift galaxies by Kobulnicky et al. (2003) have $\log L(\mathrm{H} \beta) \sim 39-43$ and 39-42, respectively, i.e. low-luminosity galaxies are lacking.

In Fig. 5 we show the oxygen abundance - absolute magnitude $M_{g}$ relation for the galaxies with oxygen abundances calculated mainly with the $T_{\mathrm{e}}$-method. In this figure, the same samples and symbols as in Fig 3 are used. The region denoted as "branch" is populated mainly by galaxies with relatively high redshifts $(z>0.02)$ and oxygen abundances derived by the $T_{\mathrm{e}^{-}}$ method. Note that selection effects could be present for "branch" high-redshift galaxies which are predominantly distant spirals. In these galaxies we select mainly low metallicity $\mathrm{H}$ II regions with a detectable [O III] $\lambda 4363$ line while the abundance gradient is present in spirals. The dotted line is a mean least-squares fit to all our data and the solid line is a mean least-squares fit to our data excluding "branch" galaxies with $M_{g}<-18.4$ and systems with an oxygen abundance in the range 8.0-8.3. The dashed line is a mean least-squares fit to the local dwarf irregular galaxies by Skillman et al. (1989). Our sample (including the SDSS subsample) shows the familiar trend of increasing metallicity with increasing luminosity. A linear least square fit to all data yields the relation

$12+\log (\mathrm{O} / \mathrm{H})=(5.706 \pm 0.199)-(0.134 \pm 0.012) M_{g}$ 

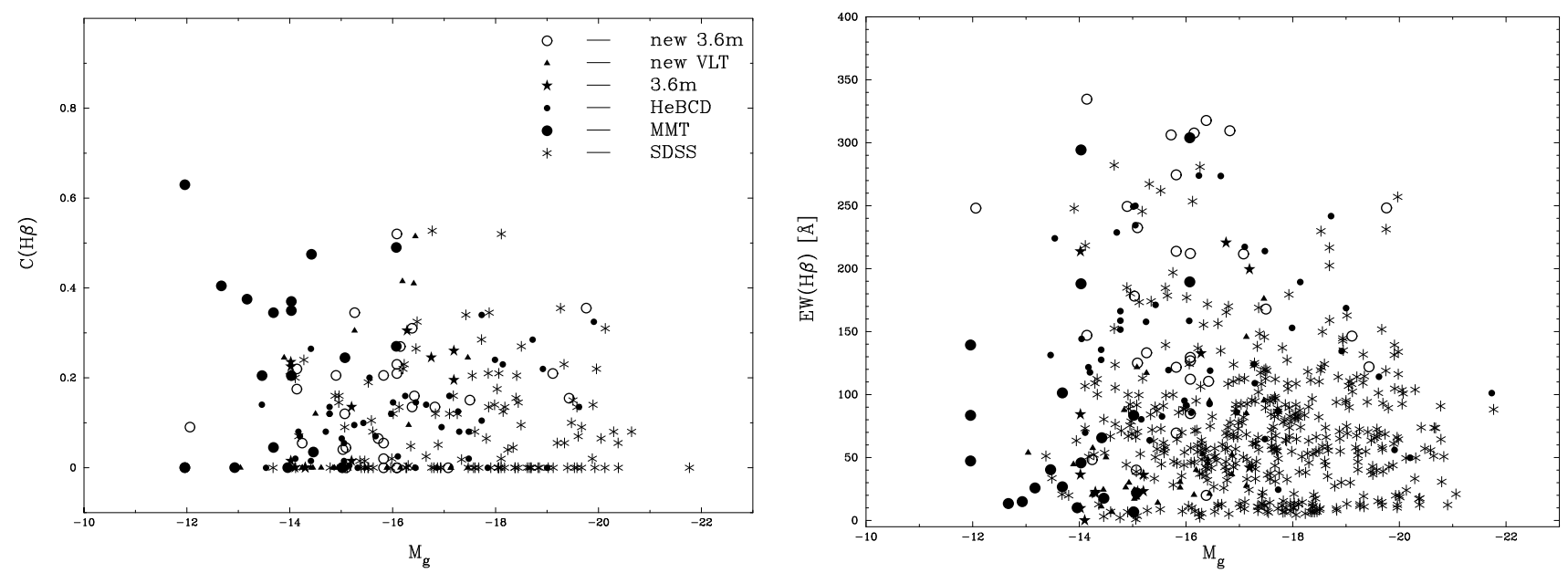

Fig. 3. The reddening parameter $C(\mathrm{H} \beta)$ obtained from the observed Balmer decrement (left) and the $\mathrm{H} \beta$ equivalent width (in $\AA$ ) (right) as a function of the absolute magnitude $M_{g}$ in the SDSS g-band. Oxygen abundances are derived mainly with the $T_{\mathrm{e}}$-method. Additionally, only high-quality SDSS spectra with the non-detected [O III] $\lambda 4363$ emission line are included for which oxygen abundances are derived with the semiempirical method (Izotov \& Thuan 2007). New $3.6 \mathrm{~m}$ ESO telescope and VLT data are shown by open circles and by stars, respectively. The additional sample of the metal-poor galaxies collected from previous $3.6 \mathrm{~m}$ ESO observations (Guseva et al. 2007) are shown by filled triangles. Filled circles denote the data from the HeBCD sample collected by Izotov et al. (2004b) and Izotov \& Thuan (2004). The MMT data from Izotov \& Thuan (2007) are shown by large filled circles. The comparison SDSS sample is represented by asterisks in which are excluded the $\mathrm{H}$ II regions in nearby spiral galaxies, faint galaxies with $m_{g}>18$, the nearest galaxies with redshift $z<0.004$ and all galaxies with $\sigma I(4363) / I(4363)>0.25$, resulting in the SDSS sample of 443 objects.

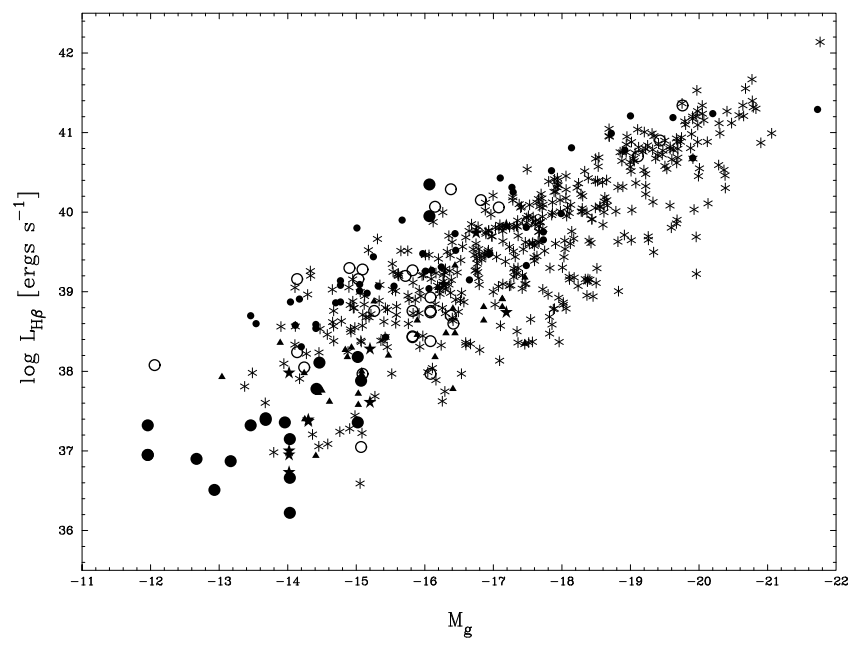

Fig. 4. The logarithm of the $\mathrm{H} \beta$ line luminosity (in $\mathrm{erg} \mathrm{s}^{-1}$ ) vs. absolute magnitude. The same samples and symbols as in Fig. 3 are used.

(dotted line in Fig. 5). Excluding "branch" galaxies we obtain the relation

$$
12+\log (\mathrm{O} / \mathrm{H})=(5.076 \pm 0.320)-(0.174 \pm 0.200) M_{g}
$$

(solid line in Fig. 5). We note that the Skillman et al. and Richer $\&$ McCall fits do not extend over the metallicity range of the present data. Therefore, we extrapolate the former fit in Fig. 5 (dashed line) to higher metallicities. Skillman's and our fits are obviosuly very similar. The slopes of our $L-Z$ relation of 0.134 (0.174) are very close to the slope of 0.153 by Skillman et al. (1989) and to the slope of 0.147 by Richer \& McCall (1995).

Our sample is well populated in the low-luminosity range, while less than 10 galaxies from the KISS sample (Salzer et al. 2005) which were used for the study of the $L-Z$ relation are fainter than $M_{B}=-15$, and none of them has an oxygen abundance less than 7.6. Our sample, excluding the SDSS subsample, has a lower dispersion around the dotted line compared to all our data and shows a shift to lower metallicities or/and higher luminosities. This likely can be attributed to our selection criteria which are optimized for the search for very metal-poor emission-line galaxies. Additionally, our sample galaxies display significant to strong ongoing SF giving rise to a large contribution from young stars and ionized gas to the total light of the galaxy. Papaderos et al. (1996, see also Papaderos et al. 2002), using surface brightness profile decomposition to separate the star-forming component from the underlying host galaxy of BCDs, found that SF regions provide on average $50 \%$ of the total $B$-band emission within the $25 B \mathrm{mag} / \square^{\prime \prime}$ isophote, with several examples of more intense starbursts whose flux contribution exceeds $70 \%$. As a result, a shift of BCDs by a $\Delta M \sim-0.75$ mag with respect to the relatively quiescent dIrr population is to be expected in Fig. 5 (see also Fig. 9). A similar offset to lower metallicities or/and higher luminosities has been found by Kakazu et al. (2007) for their intermediate-redshift low-metallicity emission-line galaxies with strong SF activity. The mass estimate of the galaxy is less sensitive to the presence of star-forming regions as compared to its luminosity. This was demonstrated by Ellison et al. (2008) who found that galaxies in close pairs show enhanced SF activity as compared to a control sample of isolated galaxies. At the same time galaxies in close pairs show a smaller offset in the mass-metallicity relation as compared to the luminosity-metallicity relation. Thus, the offset in Fig. 5 indicates that both higher luminosities and lower metallicities may contribute to the shift in the luminosity-metallicity diagram of our sample galaxies relative to more quiescent dIrrs.

In Fig. 6 we demonstrate that the region of "branch" galaxies is populated mainly by relatively high-redshift systems. The sample is the same as in Fig. 5 but in the left panel only SDSS galaxies with oxygen abundances derived with the $T_{\mathrm{e}}$-method are shown and in the right panel only relatively high-redshift galaxies with $z>0.02$ are selected.

The location of the galaxies on the luminosity-metallicity diagram is also sensitive to the method used for the abundance 


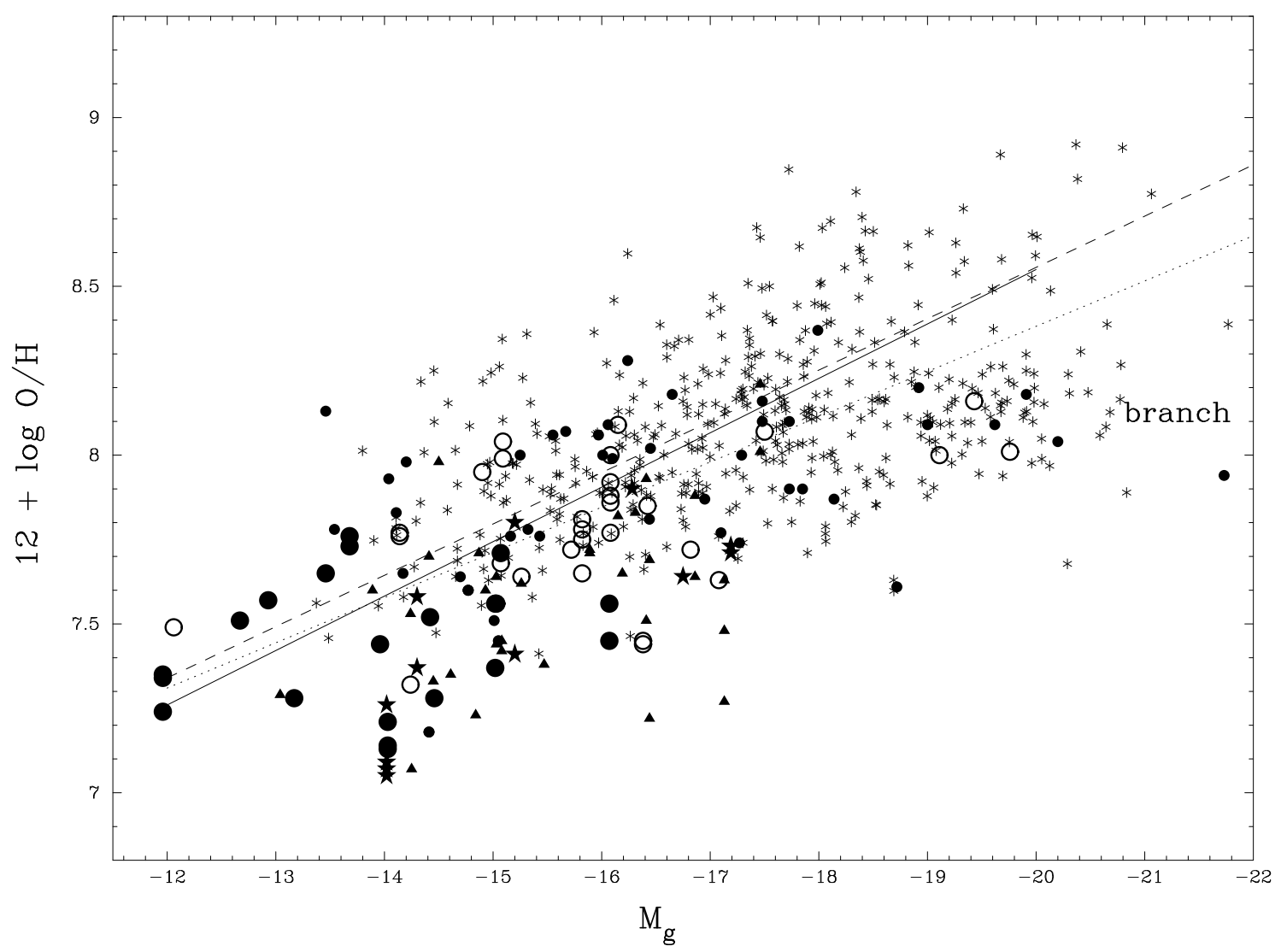

Fig. 5. Oxygen abundance vs. absolute magnitude for a large galaxy sample. The same samples and symbols as in Fig. 3 are used. The region denoted as a "branch" is populated mainly by the galaxies with relatively high redshifts $(z>0.02)$ and oxygen abundances derived by the $T_{\mathrm{e}}$ method. The dotted line is a mean least-squares fit to all our data, while the solid line is a mean least-squares fit to our data excluding "branch" galaxies with $M_{g}<-18.4$ and oxygen abundances in the range 8.0-8.3. The dashed line is a mean least-squares fit to the local dwarf irregular galaxies by Skillman et al. (1989).

determination. In order to illustrate its effect on the observed $L-Z$ relation, we compare in Fig. 7 the oxygen abundance of SDSS sample galaxies (dots) obtained with the direct $T_{\mathrm{e}}$-method (left panel) and with the semiempirical strong-line method (right panel). The abundances for other galaxies in Fig. 7 are the same as in Fig. 5. In this figure we show the larger control sample of the SDSS $(N=7964)$ as compared to Fig. 5. Only H II regions in nearby spiral galaxies and from the nearest SDSS galaxies with redshifts $z<0.004$ were excluded from the $\sim 9000$ SDSS sources while faint galaxies with $m_{g}>18$ are included. Symbols in Fig. 7 are the same as in Fig. 3 except for SDSS galaxies which are shown by dots. The dotted line is a mean least-squares fit to all our data from Fig. 5, while the solid line is a mean leastsquares fit to the same data excluding "branch" galaxies.

It can be seen from Fig. 7 that the oxygen abundance of a given galaxy obtained by different methods could differ by $\sim 0.3-0.5 \mathrm{dex}$, especially for luminous galaxies. This figure illustrates clearly above $12+\log \mathrm{O} / \mathrm{H} \sim 8.5$ and $M_{g}<-19--20$ significant discrepancies between oxygen abundances obtained from the $T_{\mathrm{e}}$-method and empirical methods. Stasińska (2002) emphasized that, at high metallicity, the $T_{\mathrm{e}}$ derived from [O III] $\lambda 4363$ would largely overestimate the temperature of the $\mathrm{O}^{++}$zone (and largely underestimate the metallicity) because cooling is dominated by the [O III] $\lambda 52 \mu \mathrm{m}$ and [O III] $\lambda 88 \mu \mathrm{m}$ lines. At the same time Pilyugin et al. (2007) demonstrated that there is an observational limit of the highest possible metallicities near $12+\log \mathrm{O} / \mathrm{H} \sim 8.95$. This maximum value was determined in the centers of the most luminous $\left(-22.3 \lessgtr M_{B} \lesssim-20.3\right)$ galaxies using the semiempirical ffmethod (Pilyugin et al. 2006). Thus, although the main mechanisms determining the electron temperature in $\mathrm{H}$ II nebulae have been known for a long time, there are still important unsolved problems.

The contribution of star-forming regions to the light of the galaxy can be quantified by the equivalent width $E W(\mathrm{H} \beta)$ of the $\mathrm{H} \beta$ emission line which in turn depends on the age of the burst of star formation. In Fig. 8 we show the same samples as in Fig. 5 except for the SDSS galaxies now being split into two subsamples. In the left panel only those with high equivalent widths $E W(\mathrm{H} \beta)>80 \AA$ are shown while in the right panel only SDSS galaxies with low equivalent widths $E W(\mathrm{H} \beta)<20 \AA$ are plotted. The dotted line in the left and right panels is a mean least-squares fit to all our data shown in Fig. 5, while the solid line is a mean least-squares fit to the same data excluding "branch" galaxies. There is a clear difference between the two subsamples of the SDSS galaxies by $\sim 0.4$ dex in oxygen abundance or, equivalently, by $\sim 3$ mag in absolute magnitude. SDSS galaxies with $E W(\mathrm{H} \beta)>80 \AA$ A nicely follow the relation for our dwarf low-metallicity emission-line galaxies shown as reference objects by filled and open circles, stars, filled triangles and large filled circles. On the other hand, the SDSS galaxies with $E W(\mathrm{H} \beta)<20 \AA$ are located systematically above the low-metallicity galaxies. We propose two possible explanations for such a difference between the two subsamples of SDSS galaxies: 1) the emission of the SDSS galaxies with high $E W(\mathrm{H} \beta)$ is dominated by star-forming regions, therefore they 

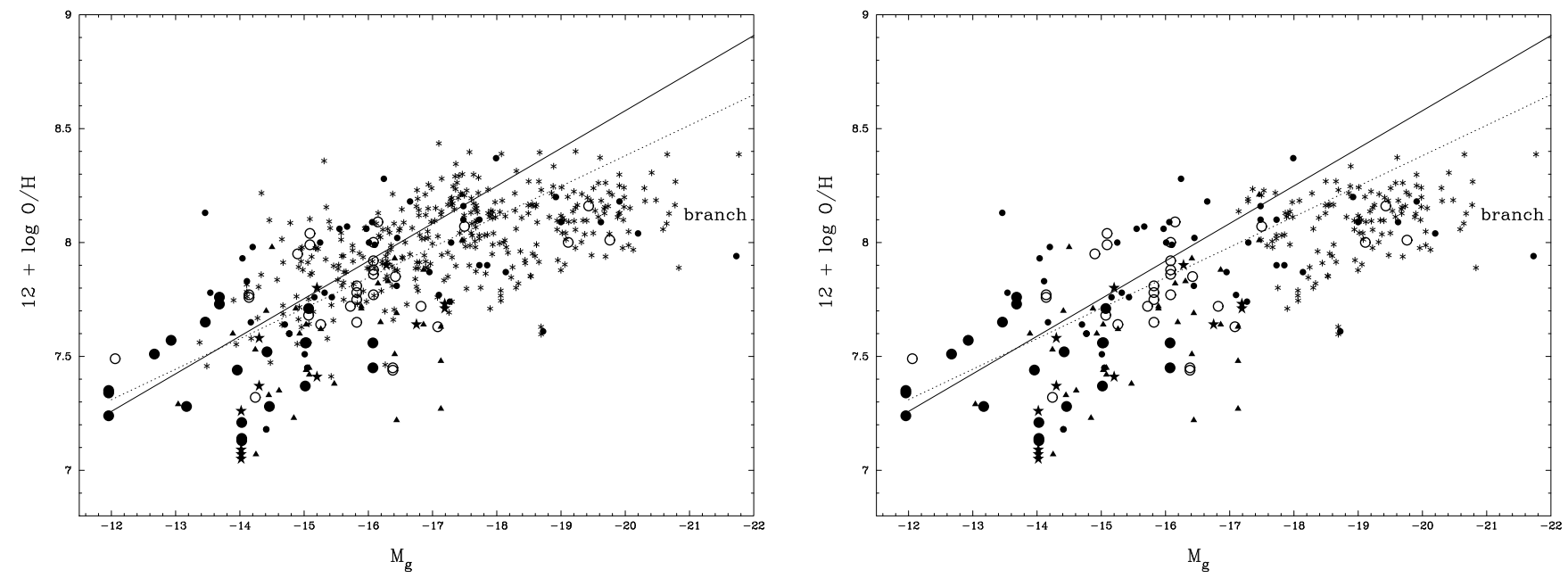

Fig. 6. The same as in Fig. 5 but (left) only SDSS galaxies with oxygen abundances derived with the $T_{\mathrm{e}}$-method and (right) only relatively highredshift galaxies with $z>0.02$. The dotted line is a mean least-squares fit to all our data, while the solid line is a mean least-squares fit to our data excluding "branch" galaxies.

have higher luminosities compared to galaxies in a relatively quiescent stage; 2) SDSS galaxies with low $E W(\mathrm{H} \beta)$ are the ones with higher astration level, therefore they are more chemically evolved systems with higher oxygen abundances. Perhaps both of these explanations are tenable, accounting for the observed differences between SDSS galaxies with high and low $E W(\mathrm{H} \beta)$. Thus, the lowest-metallicity SDSS galaxies are found predominantly among galaxies with high $E W(\mathrm{H} \beta)$. On the other hand, no extremely low-metallicity SDSS galaxies are found among systems with $E W(\mathrm{H} \beta)<20 \AA$. Thus, mixing of the SDSS galaxies with $E W(\mathrm{H} \beta)<20 \AA$ and $>80 \AA$ results in a significant increase of the dispersion of the luminosity-metallicity diagram.

The redshift of the galaxy could also play a role. In Fig. 5 the bulk of the galaxies with $12+\log (\mathrm{O} / \mathrm{H})=8.0-8.3$ and absolute magnitudes between -19 and -21 mag (denoted as "branch" galaxies) is represented by higher-redshift systems as compared to other galaxies from the SDSS and a correction for redshift is required. Since "branch" galaxies are blue, a correction for redshift for systems with weak emission lines would increase their brightness by $\sim 0.1-0.3 \mathrm{mag}$. This will not be enough to remove the offset between "branch" galaxies and lower-redshift galaxies in Fig. 5. The situation is more complicated for "branch" galaxies with strong emission lines since their effect on the apparent magnitudes of a galaxy in standard passbands will significantly depend on redshift (see e.g. Zackrisson et al. 2008). Because of these reasons, we decided not to take into account corrections for redshift.

\subsection{Comparison of our sample with other data}

In Fig. 9 we compare our $L-Z$ relation with other published data for galaxies of different types. In this figure, all of our galaxies from Fig. 5, including those from the comparison SDSS sample, are shown by small filled and open circles. Some well known metal-poor galaxies are depicted by large filled circles and are labelled. Their absolute magnitudes $M_{B}$ are taken from Kewley et al. (2007). For comparison, 23 KISS emission-line galaxies by Lee et al. (2004) are displayed with large open double circles. The abundances for these galaxies are derived with the $T_{\mathrm{e}}$-method, the $B$-band magnitudes are from Salzer et al. (1989) and Gil de Paz et al. (2003). With open double squares we show 25 nearby dIrrs with the $4.5 \mu \mathrm{m}$ Spitzer luminosities and compiled $\mathrm{O} / \mathrm{H}$ abundances derived with the $T_{\mathrm{e}}$-method (Lee et al. 2006). With large open circles and large crosses we respectively show 20 irregular galaxies from Skillman et al. (1989) and 21 dwarf irregular galaxies from Richer \& McCall (1995) for which oxygen abundances are obtained mainly with the $R_{23} \mathrm{em}-$ pirical method, and for a few objects only with the $T_{\mathrm{e}}$-method. The thick solid line is a mean least-squares fit to all our data. The thin solid line is a least-squares fit to the data by Richer \& McCall (1995) while the dotted line is a mean least-squares fit to the data by Skillman et al. (1989). The dashed line is the luminosity-metallicity relation for local metal-poor BCDs obtained by Kunth \& Östlin (2000).

Data for intermediate- and high-redshift galaxies are also shown. The most distant $(z<1)$ extremely metal-poor galaxies (XMPGs) (Kakazu et al. 2007) with the oxygen abundances derived with the empirical method are shown with filled squares, while relatively metal-poor luminous galaxies at $z \sim 0.7$ (Hoyos et al. 2005) $\left(\mathrm{O} / \mathrm{H}\right.$ derived with the $T_{\mathrm{e}}$-method) with filled triangles. The remaining samples in Fig. 9 are the following: a) the large open circles correspond to the $z=3.36$ lensed galaxy (Villar-Martín et al. 2004) and to the average position of luminous Lyman-break galaxies at redshifts $z \sim 2.5$ (Kobulnicky \& Koo 2000) (O/H derived with the $R_{23}$ method); b) small open circles stand for 66 Canada-France Redshift Survey (CFRS) galaxies by Lilly et al. (2003) in the redshift range of $\sim 0.5-1.0\left(\mathrm{O} / \mathrm{H}\right.$ derived with the $R_{23}$ empirical method); c) asterisks are for 204 GOODS-N (Great Observatories Origins Deep Survey - North) emission-line galaxies in the range of redshifts $0.3<z<1.0$ (Kobulnicky \& Kewley 2004) $(\mathrm{O} / \mathrm{H}$ is derived with the $R_{23}$ empirical method); d) small open rombs indicate 64 emission-line field galaxies from the Deep Extragalactic Evolutionary Probe Groth Strip Survey (DGSS) in the redshift range of $\sim 0.3-0.8$ (Kobulnicky et al. 2003) (O/H derived with the $R_{23}$ empirical method); e) open squares are for the gammaray burst (GRB) hosts by Kewley et al. (2007). Small open squares are for galaxies with $\mathrm{O} / \mathrm{H}$ derived with the empirical method (Kewley \& Dopita 2002) and large open squares for the galaxies with $\mathrm{O} / \mathrm{H}$ derived with the $T_{\mathrm{e}}$-method (Kewley et al. 2007); f) filled stars denote the 14 star-forming emissionline galaxies at intermediate redshifts $(0.11<z<0.5)$ by Kobulnicky \& Zaritsky (1999) (O/H derived with the empirical $R_{23}$ method); g) open triangles are for 29 distant $15 \mu$ m-selected 

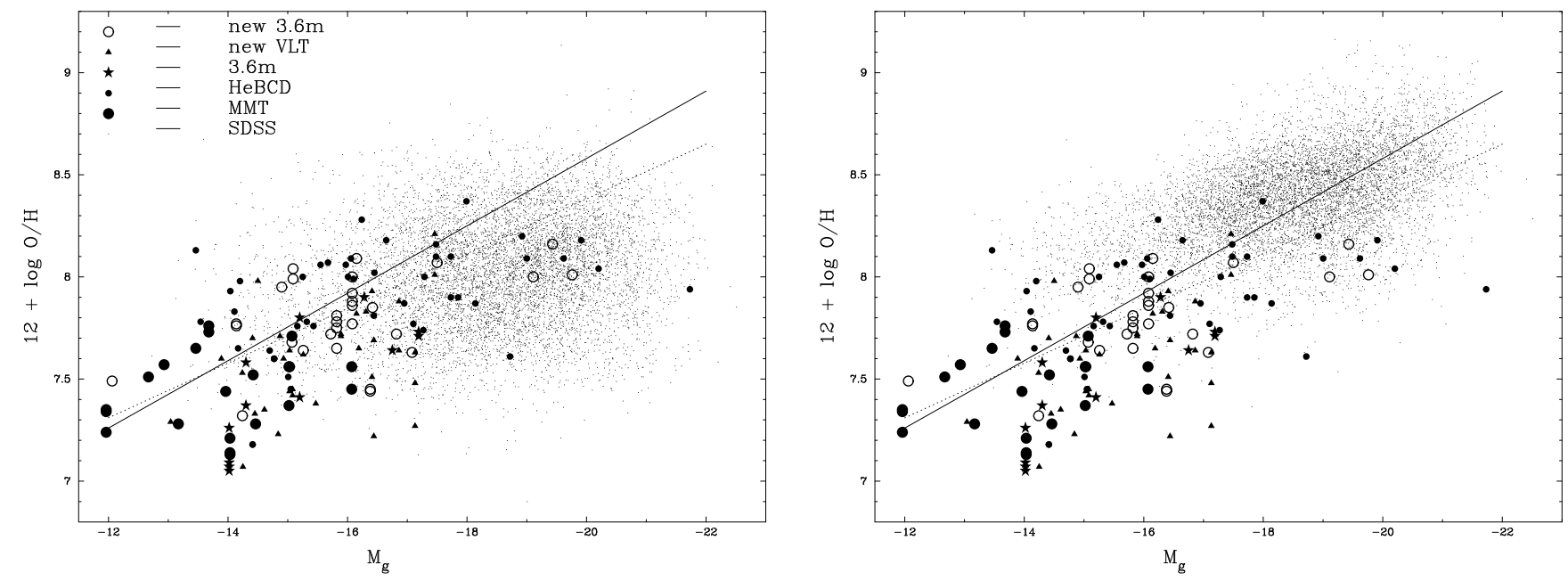

Fig. 7. Oxygen abundance vs. absolute magnitude. H II regions in spiral galaxies and the nearest SDSS galaxies with redshift $z<0.004$ are excluded from the SDSS sample while faint galaxies with $m_{g}>18$ are included, resulting in an SDSS sample of 7964 objects. Symbols are the same as in Fig. 3 except for SDSS galaxies which are shown by dots. In the left panel the oxygen abundances $12+\log \mathrm{O} / \mathrm{H}$ for SDSS galaxies are obtained with the $T_{\mathrm{e}}$-method. In the right panel $12+\log \mathrm{O} / \mathrm{H}$ for SDSS galaxies is derived with the semiempirical method. The dotted line is a mean least-squares fit to all our data from Fig. 5, while the solid line is a mean least-squares fit to the same data excluding "branch" galaxies.
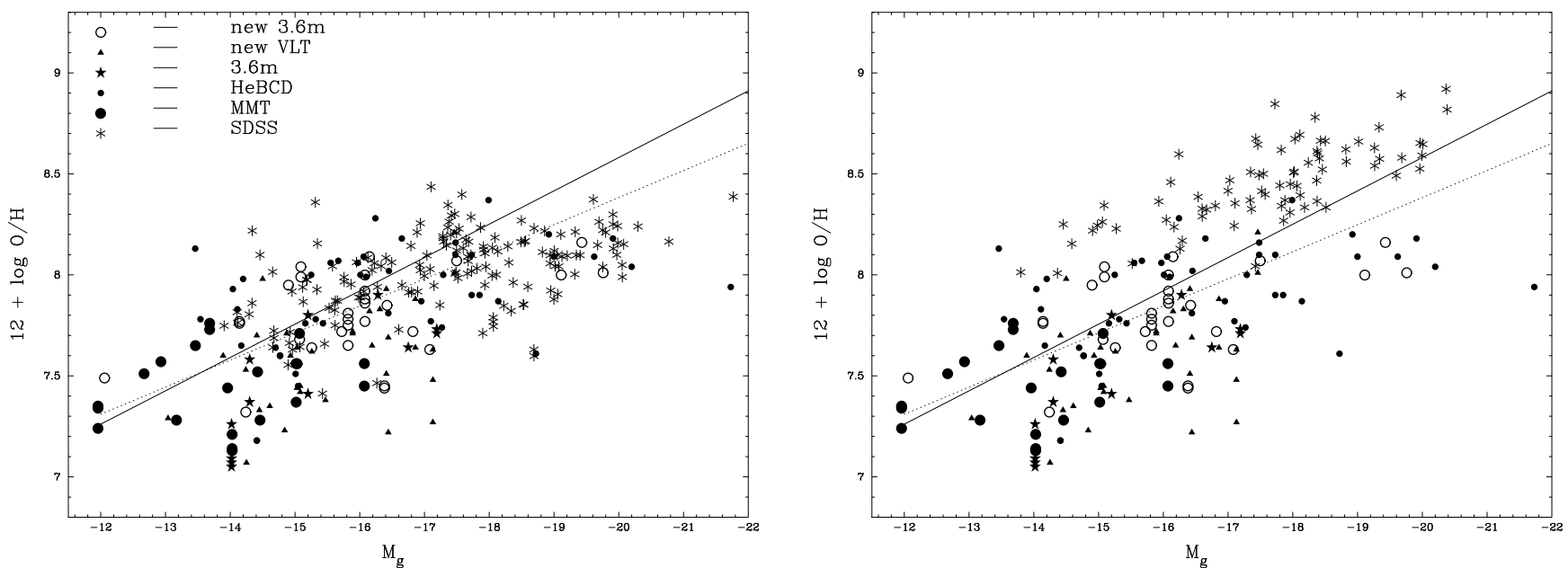

Fig. 8. The same as in Fig. 5 but only SDSS galaxies with $E W(\mathrm{H} \beta)>80 \AA$ (left panel) and with $E W(\mathrm{H} \beta)<20 \AA$ (right panel). The dotted line is a mean least-squares fit to all our data from Fig. 5, while the solid line is a mean least-squares fit to the same data excluding "branch" galaxies. For the SDSS galaxies with $E W(\mathrm{H} \beta)>80 \AA$ the oxygen abundances are $\sim 0.4$ dex lower than the ones for the galaxies with $E W(\mathrm{H} \beta)<20 \AA$. The galaxies with the lowest metallicities could be found more easily among the $\mathrm{H}$ II regions with high $E W(\mathrm{H} \beta)$.

luminous infrared galaxies (LIRGs) at $z \sim 0.3-0.8$ taken from the sample of Liang et al. (2004) (O/H derived with the empirical method); and, finally; h) the large dotted rectangle depicts the position of Lyman break galaxies (LBGs, Pettini et al. 2001) on the $L-Z$ diagram.

The location of our galaxies on the luminosity-metallicity diagram is similar to that obtained previously for local emissionline galaxies but is shifted to higher luminosities and/or lower metallicities compared to that obtained for quiescent irregular dwarf galaxies. For comparison, Lee et al. (2004) have also demonstrated that their $54 \mathrm{H}$ II KISS galaxies with $\mathrm{O} / \mathrm{H}$ derived with the $T_{\mathrm{e}}$-method follow the $L-Z$ relation with a slope similar to that for a more quiescent dIrrs but are shifted to higher brightness by 0.8 mag. Furthermore, they have shown that $\mathrm{H}$ II galaxies with disturbed irregular outer isophotes (likely due to the interaction) are shifted to a more luminous and/or more metal-poor region in the $L-Z$ diagram as compared to morphologically more regular galaxies. Note that their samples of $\mathrm{H}$ II galaxies and of
dIrrs are in the same luminosity range as our sample. Papaderos et al. (2008) also note that in contrast to the majority (>90\%) of BCDs, the extremely metal-poor SF dwarfs reveal more irregular and bluer hosts.

Thus, the difference in the zero point between our $L-Z$ relation for low-metallicity galaxies and for other galaxies seems to be primarily due to the differences in the intrinsic properties of the galaxies selected for different samples with various selection criteria.

A key question is whether a unique $L-Z$ relation does exist for galaxies of different types. The assessment of this issue is complicated by offsets of high-redshift galaxies with different look-back-times. In this context, Kobulnicky et al. (2003) have shown that both the slopes and zero points of the $L-Z$ relation exhibit a smooth evolution with redshift. A possible universal $L-Z$ relation for galaxies is also blurred by the fact that metallicity determinations of various galaxy samples, differing in their $E W(\mathrm{H} \beta)$, absolute magnitude and redshift, do not employ a 


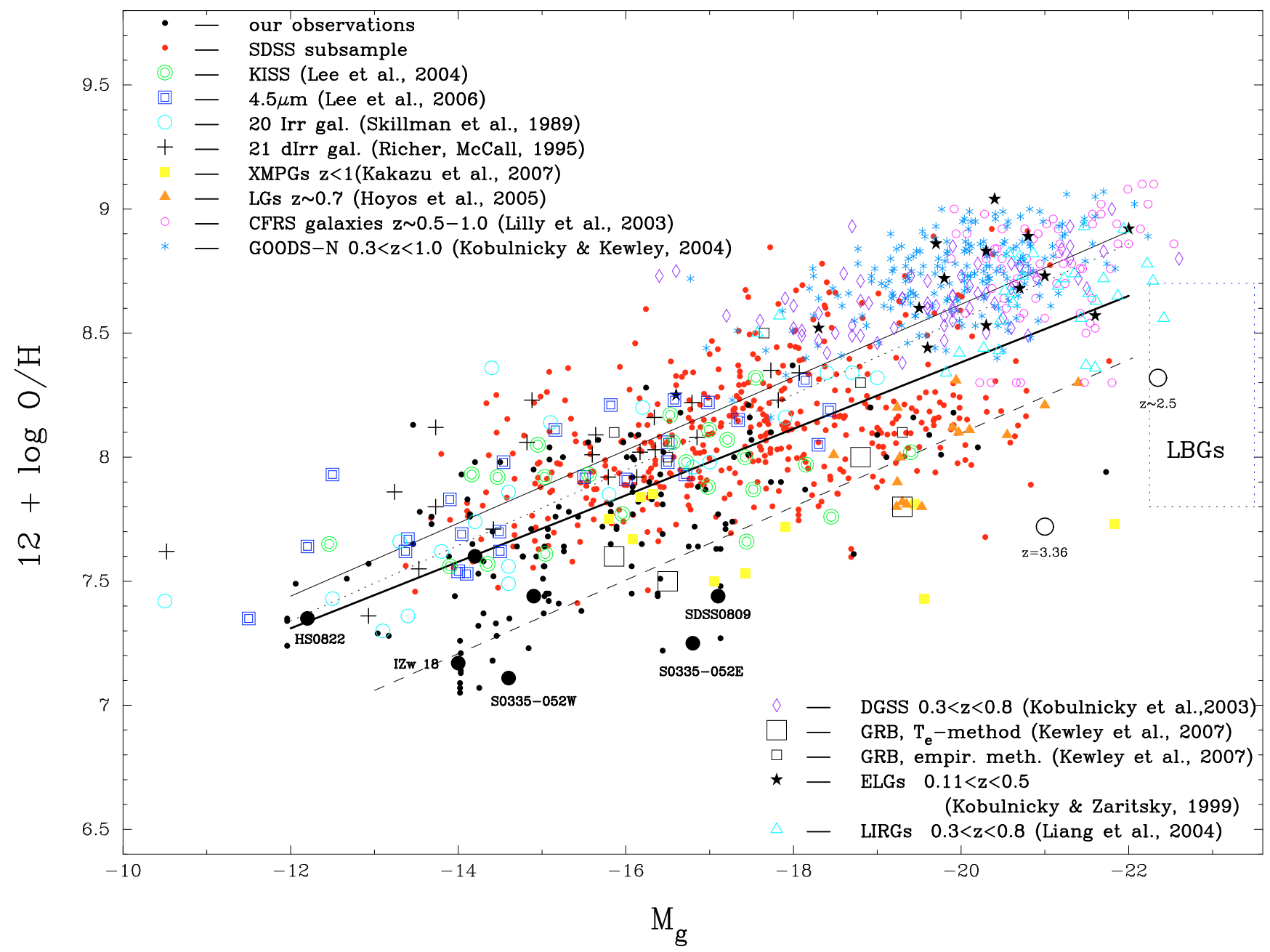

Fig. 9. Oxygen abundance vs. absolute magnitude for the same galaxies as in Fig. 5 but all our galaxies including the SDSS sample are shown by small filled circles. Additionally, some well known metal-poor galaxies, intermediate- and high-redshift galaxies are shown by symbols as labelled in the figure. The position of the Lyman break galaxies (LBGs) by Pettini et al. (2001) is indicated by the dotted line rectangle. The thick solid line is a mean least-squares fit to all our data. The thin solid line is a least-squares fit to the data by Richer \& McCall (1995) while the dotted line is a mean least-squares fit to the data by Skillman et al. (1989). The dashed line is the luminosity-metallicity relation for local metal-poor BCDs obtained by Kunth \& Östlin (2000).

unique technique. More specifically, several authors emphasize the presence of a well-known shift between the $\mathrm{O} / \mathrm{H}$ ratio obtained by the direct $T_{\mathrm{e}}$-method and empirical strong-line methods. Oxygen abundances obtained by empirical methods are by 0.1-0.25 dex (Shi et al. 2005) and even by up to 0.6 dex (Hoyos et al. 2005) higher than those obtained with the $T_{\mathrm{e}}$-method. For our sample we obtained an offset of $\sim 0.3-0.5$ dex.

It can be seen from Fig. 9 that the high-redshift galaxies with an oxygen abundance derived by the $T_{\mathrm{e}}$-method have a shallower slope compared to local galaxies. On the other hand, oxygen abundances of high-redshift galaxies obtained with the $R_{23}$ empirical strong-line method (data in Fig. 9 by Lilly et al. 2003; Kobulnicky \& Kewley 2004; Liang et al. 2004) are higher and follow the relation for high-metallicity SDSS galaxies in Fig. 7b despite the fact that oxygen abundances for the latter galaxies were calculated with the different semi-empirical strongline method. Because of this agreement we decided not to recalculate oxygen abundances of high-redshift galaxies with the semi-empirical method and adopted $\mathrm{O} / \mathrm{H}$ values from the literature. Keeping in mind the systematic differences between oxygen abundances derived with the empirical and the $T_{\mathrm{e}}$-methods, it might be worth considering a decrease in oxygen abundance by $\sim 0.2-0.6$ dex for all high-redshift galaxies with $\mathrm{O} / \mathrm{H}$ derived with the empirical method. In that case, the position of highredshift galaxies on the $L-Z$ diagram would be consistent with that of the "branch" galaxies. Such considerations add further support to the results obtained by Pilyugin et al. (2004) and Shi et al. (2005) that the more luminous galaxies have a slope of the $L-Z$ relation more shallow than that of the dwarf galaxies.

We presume that our $L-Z$ relation could be useful as a local reference for studies of this relation for other types of local galaxies and/or of high-redshift galaxies.

\section{Summary}

We present VLT spectroscopic observations of a new sample of $28 \mathrm{H}$ II regions from 16 emission-line galaxies and ESO $3.6 \mathrm{~m}$ telescope spectroscopic observations of a new sample of $38 \mathrm{H}$ II regions from 28 emission-line galaxies. These galaxies have mainly been selected from the data release 6 (DR6) of the Sloan digital sky survey (SDSS) as low-metallicity galaxy candidates. 
Physical conditions and element abundances are derived for $38 \mathrm{H}$ II regions observed with the $3.6 \mathrm{~m}$ telescope and for $23 \mathrm{H}$ II regions observed with the VLT.

From our new observations we find that the oxygen abundance in 61 out of the 66 observed $\mathrm{H}$ II in our sample ranges from $12+\log \mathrm{O} / \mathrm{H}=7.05$ to 8.22 . The oxygen abundance in $27 \mathrm{H}$ II regions is $12+\log \mathrm{O} / \mathrm{H}<7.6$ and among them $10 \mathrm{H}$ II regions have an oxygen abundance less than 7.3.

This new data in combination with objects from our previous studies constitute a large uniform sample of $154 \mathrm{H}$ II regions with high-quality spectroscopic data which are used to study the luminosity-metallicity $(L-Z)$ relation for the local galaxies with emphasis on its low-metallicity end.

As a comparison sample we use $\sim 9000$ SDSS emission-line galaxies with higher oxygen abundances which are also obtained mainly by the direct $T_{\mathrm{e}}$-method. For all of our sample galaxies the $g$ magnitudes are taken from the SDSS while the distances are from the NED. The entire sample spans nearly two orders of magnitude with respect to its gas-phase metallicity, from $12+$ $\log \mathrm{O} / \mathrm{H} \sim 7.0$ to $\sim 8.8$, and covers an absolute magnitude range from $M_{g} \sim-12$ to $\sim-20$.

We find that the metallicity-luminosity relation for our galaxies is consistent with previous ones obtained for objects of similar type. The local $L-Z$ relation obtained with high-quality spectroscopic data is useful for predictions of galaxy evolution models.

Acknowledgements. N. G. G. and Y. I. I. thank the Max Planck Institute for Radioastronomy (MPIfR) for hospitality, and acknowledge support through DFG grant No. Fr 325/57-1. P. P. thanks the Department of Astronomy and Space Physics at Uppsala University for its warm hospitality. K. J. Fricke thanks the MPIfR for Visiting Contracts during 2008 and 2009. This research was partially funded by project grant AYA2007-67965-C03-02 of the Spanish Ministerio de Ciencia e Innovacion. We acknowledge the work of the Sloan digital sky survey (SDSS) team. Funding for the SDSS has been provided by the Alfred P. Sloan Foundation, the Participating Institutions, the National Aeronautics and Space Administration, the National Science Foundation, the US Department of Energy, the Japanese Monbukagakusho, and the Max Planck Society. The SDSS Web site is http: //www.sdss.org/

\section{References}

Abazajian, K. N., Adelman-McCarthy, J. K., Agüeros, M. A., et al. 2009, ApJS, 182,543

Contini, T., Treyer, M. A., Sullivan, M., \& Ellis, R. S. 2002, MNRAS, 330, 75

Ellison, S. L., Patton, D. R., Simard, L., \& McConnachie, A. W. 2008, AJ, 135, 1877

Gavilán, M., Mollá, M., \& Díaz, Á. 2009, to appear in Star-forming Dwarf Galaxies: Following Ariadnes Thread in the Cosmic Labyrinth, [arXiv: 0902 . 4695]

Gil de Paz, A., Madore, B. F., \& Pevunova, O. 2003, ApJS, 147, 29

Guseva, N. G., Izotov, Y. I., Papaderos, P., \& Fricke, K. J. 2007, A\&A, 464, 885
Hoyos, C., Koo, D. C., Phillips, A. C., Willmer, C. N. A., \& Guhathakurta, P. 2005, ApJ, 635, L21

Izotov, Y. I., \& Thuan, T. X. 2004, ApJ, 602, 200

Izotov, Y. I., \& Thuan, T. X. 2007, ApJ, 665, 1115

Izotov, Y. I., Lipovetsky, V. A., Chaffee, F. H., et al. 1997, ApJ, 476, 698

Izotov, Y. I., Noeske, K. G., Guseva, N. G., et al. 2004a, A\&A, 415, L27

Izotov, Y. I., Stasińska, G., Guseva, N. G., \& Thuan, T. X. 2004b, A\&A, 415, 87

Izotov, Y. I., Stasińska, G., Meynet, G., Guseva, N. G., \& Thuan, T. X. 2006a, A\&A, 448, 955

Izotov, Y. I., Papaderos, P., Guseva, N. G., Fricke, K. J., \& Thuan, T. X. 2006b, A\&A, 454, 137

Kakazu, Y., Cowie, L. L., \& Hu, E. M. 2007, ApJ, 668, 853

Kewley, L. J., \& Dopita, M. A. 2002, ApJS, 142, 35

Kewley, L. J., Brown, W. R., Geller, M. J., Kenyon, S. J., \& Kurtz, M. J. 2007, AJ, 133, 882

Kobulnicky, H. A., \& Zaritsky, D. 1999, ApJ, 511, 118

Kobulnicky, H. A., \& Koo, D. C. 2000, ApJ, 545, 712

Kobulnicky, H. A., \& Kewley, L. J. 2004, ApJ, 617, 240

Kobulnicky, H. A., Willmer, C. N. A., Phillips, A. C., et al. 2003, ApJ, 599, 1006

Kunth, D., \& Östlin, G. 2000, Astron. Astrophys. Rev., 10, 1

Lamareille, F., Mouhcine, M., Contini, T., Lewis, L., \& Maddox, S. 2004, MNRAS, 350, 396

Lee, H., Skillman, E. D., Cannon, J. M., et al. 2006, ApJ, 647, 970

Lee, J. C., Salzer, J. J., \& Melbourne, J. 2004, ApJ, 616, 752

Lequeux, J., Rayo, J. F., Serrano, A., Peimbert, M., \& Torres-Peimbert, S. 1979, A\&A, 80, 155

Liang, Y. C., Hammer, F., Flores, H., et al. 2004, A\&A, 423, 867

Lilly, S. J., Carollo, C. M., \& Stockton, A. N. 2003, ApJ, 597, 730

Maier, C., Meisenheimer, K., \& Hippelein, H. 2004, A\&A, 418, 475

Melbourne, J., \& Salzer, J. J. 2002, AJ, 123, 2302

Noeske, K. G., Papaderos, P., Cairos, L. M., \& Fricke, K. J. 2003, A\&A, 410, 481

Papaderos, P., Loose, H.-H., Fricke, K. J., \& Thuan, T. X. 1996, A\&A, 314, 59

Papaderos, P., Izotov, Y. I., Fricke, K. J., Thuan, T. X., \& Guseva, N. G. 1998, A\&A, 338, 43

Papaderos, P., Izotov, Y. I., Thuan, T. X., et al. 2002, A\&A, 393, 461

Papaderos, P., Guseva, N. G., Izotov, Y. I., \& Fricke, K. J. 2008, A\&A, 491, 113

Pettini, M., Shapley, A. E., Steidel, C. C., et al. 2001, ApJ, 554, 981

Pilyugin, L. S., Vílchez, J. M., \& Contini, T. 2004, A\&A, 425, 849

Pilyugin, L. S., Thuan, T. X., \& Vílchez, J. M. 2006, MNRAS, 367, 1139

Pilyugin, L. S., Thuan, T. X., \& Vílchez, J. M. 2007, MNRAS, 376, 353

Richer, M. G., \& MacCall, M. L. 1995, ApJ, 445, 642

Salzer, J. J., MacAlpine, G. M., \& Boroson, T. A. 1989, ApJS, 70, 479

Salzer, J. J., Lee, J. C., Melbourne, J., et al. 2005, ApJ, 624, 661

Saviane, I., Ivanov, V. D., Held, E. V., et al. 2008, A\&A, 487, 901

Shi, F., Kong, X., Li, C., \& Cheng, F. Z. 2005, A\&A, 437, 849

Skillman, E. D., Kennicutt, R. C., Jr., \& Hodge, P. W. 1989, ApJ, 347, 875

Smith, B. J., \& Hancock, M. 2009, AJ, 138, 130

Stasińska, G. 2002, RMxAC, 12, 62

Stasińska, G., \& Izotov, Y. I. 2003, A\&A, 397, 71

Thuan, T. X., \& Izotov, Y. I. 2005, ApJS, 161, 240

Tremonti, C. A., Heckman, T. M., Kauffmann, G., et al. 2004, ApJ, 613, 898

Vanzi, L., Hunt, L. K., Thuan, T. X., \& Izotov, Y. I. 2000, A\&A, 363, 493

Vaduvescu, O., McCall, M. L., \& Richer, M. G. 2007, AJ, 134, 604

Vila-Costas, M. B., \& Edmunds, M. G. 1992, MNRAS, 259, 121

Villar-Martín, M., Cerviño, M., \& González Delgado, R. M. 2004, MNRAS, 355,1132

Whitford, A. E. 1958, AJ, 63, 201

Zackrisson, E., Bergvall, N., \& Leitet, E. 2008, ApJ, 676, L9 
N. G. Guseva et al.: The L-Z relation for a large sample of low-metallicity galaxies, Online Material p 1

Table 1. List of galaxies observed with the $3.6 \mathrm{~m}$ ESO telescope.

\begin{tabular}{|c|c|c|c|c|c|c|c|}
\hline Galaxy & & $\begin{array}{l}\text { RA, Dec. } \\
\text { (J2000) }\end{array}$ & $\begin{array}{c}\text { Date } \\
(2007)\end{array}$ & $\begin{array}{l}\text { Exp. time } \\
\text { (s) }\end{array}$ & Airmass & Seeing & Redshift \\
\hline J0015+0104 & $00^{\mathrm{h}} 15^{\mathrm{n}}$ & ${ }^{\mathrm{n}} 20^{\mathrm{s}} 7, \quad 01^{\circ} 04^{\prime} 37^{\prime \prime}$ & 14 Sep. & $4 \times 900$ & 1.207 & 1.74 & $0.00689 \pm 0.00021$ \\
\hline J0016+0108 & 0016 & 28. 3, 010802 & 15 Sep. & $4 \times 675$ & 1.306 & 1.90 & $0.01035 \pm 0.00006$ \\
\hline J0029-0108 & 0029 & $04.7,-010826$ & 16 Sep. & $4 \times 450$ & 1.614 & 1." 88 & $0.01313 \pm 0.00006$ \\
\hline J0029-0025 & 0029 & 49. $5,-002540$ & 14 Sep. & $4 \times 900$ & 1.142 & 1."72 & $0.01440 \pm 0.00013$ \\
\hline 0057-0022 & 0057 & 12. $6,-002158$ & 16 Sep. & $4 \times 900$ & 1.525 & $1 . " 47$ & $0.00956 \pm 0.00023$ \\
\hline $\mathrm{J} 0107+0001$ & 0107 & $50.8, \quad 0001 \quad 28$ & 16 Sep. & $3 \times 267$ & 1.188 & 1." 41 & $0.01835 \pm 0.00012$ \\
\hline J0109+0107 & 0109 & $08.0, \quad 0107 \quad 16$ & 15 Sep. & $4 \times 900$ & 1.174 & $1 . " 54$ & $0.00396 \pm 0.00013$ \\
\hline J0126-0038 No. 1 & 0126 & 46. 1, -00 3839 & 16 Sep. & $4 \times 900$ & 1.332 & 1."36 & $0.00632 \pm 0.00010$ \\
\hline J0126-0038 No. 2 & 0126 & 46. $1,-003839$ & 16 Sep. & $4 \times 900$ & 1.332 & $1 " 36$ & $0.00642 \pm 0.00016$ \\
\hline J0135-0023 & 0135 & 44. $0,-002317$ & 14 Sep. & $4 \times 900$ & 1.142 & 2.33 & $0.01708 \pm 0.00006$ \\
\hline J0213-0002 No. 1 & $02 \quad 13$ & $57.7,-000256$ & 16 Sep. & $4 \times 900$ & 1.252 & $1 . " 52$ & $0.03640 \pm 0.00008$ \\
\hline J0213-0002 No. 2 & 0213 & $57.7,-000256$ & 16 Sep. & $4 \times 900$ & 1.252 & $1 . " 52$ & $0.03635 \pm 0.00011$ \\
\hline J0216+0115 No. 1 & 0216 & 29. 3,-01 1521 & 15 Sep. & & 1.223 & $2 . .02$ & $0.00939 \pm 0.00004$ \\
\hline J0216+0115 No. 2 & 0216 & 29. 3, $01 \quad 1521$ & 15 Sep. & $4>$ & 1.223 & 2.02 & $0.00940 \pm 0.00004$ \\
\hline 096632 & 0251 & 47. $5,-300632$ & 15 Sep. & $4 \times$ & 1.400 & 2.28 & 0.00354 \\
\hline $\mathrm{J} 0252+0017$ & 0252 & 16. $8,00 \quad 1741$ & 16 Sep. & & 1.165 & 1". 38 & $0.00527=$ \\
\hline $\mathrm{J} 0256+0036$ & 0256 & $28.3,003628$ & 14 Sep. & $4 \times 900$ & 1.150 & $2 ! .47$ & $0.00919 \pm 0.00013$ \\
\hline J0303-0109 No. 1 & 0303 & $31.3,-010947$ & 14 Sep. & $2>$ & 70 & 2.43 & $0.03055 \pm 0.00011$ \\
\hline J0303-0109 No. 2 & 0303 & $31.3,-010947$ & 14 Sep. & & & $2 ! .43$ & $0.03039=$ \\
\hline J0341-0026 I & 0341 & 18. $1,-002628$ & 16 Sep. & $3 x$ & 1.198 & 1.. 46 & $0.03080 \pm 0.00020$ \\
\hline J0341-0026 No. 2 & 0341 & 18. 1,-00 2628 & $16 \mathrm{Sep}$ & $3 \times$ & 1.198 & 1." 46 & $0.03045 \pm 0.00008$ \\
\hline J0341-0026 No. 3 & 0341 & 18. 1,-00 2628 & 16 Sep. & & & 1.. 46 & $0.03047 \pm 0.00008$ \\
\hline G1815456-6 & 1815 & $46.5,-670123$ & 14 Sep. & 4 & 1.280 & $2 \prime .00$ & $0.01131 \pm 0.00008$ \\
\hline G2052078-691229 No. 1 & 2052 & $07.1,-691230$ & 16 Sep. & $3 \times 800$ & 1.328 & $1 . " 50$ & $0.00212 \pm 0.00008$ \\
\hline G2052078-691229 No. 2 & 2052 & 07. 1, -69 1230 & 16 Sep. & & & $1 " .50$ & $0.00203 \pm 0.00011$ \\
\hline $\mathrm{J} 2053+0039$ & 2053 & 12. $6, \quad 003915$ & 15 Sep. & & 1.219 & $1^{\prime \prime} .55$ & $0.01328 \pm 0.00007$ \\
\hline J2105+0032 No. 1 & 2105 & $08.6, \quad 0032 \quad 23$ & 14 Sep. & $3 \times 800$ & 1.408 & 1 1. 45 & $0.01431 \pm 0.00003$ \\
\hline J2105+0032 No. 2 & 2105 & $08.6, \quad 003223$ & 14 Sep. & $3 \times 800$ & 1.408 & $1 . .45$ & $0.01436 \pm 0.00013$ \\
\hline J2112-0016 No. 1 & 2112 & $00.8,-001649$ & 15 Sep. & $3 \times 800$ & 1.441 & 1.'32 & $0.01195 \pm 0.00015$ \\
\hline J2112-0016 No. 2 & 2112 & $00.8,-001649$ & 15 Sep. & $3 \times 800$ & 1.441 & 1.32 & $0.01215 \pm 0.00021$ \\
\hline J2119-0732 & 2119 & $42.4,-073224$ & 14 Sep. & $3 \times 800$ & 1.130 & $1{ }^{\prime \prime} 78$ & $0.00966 \pm 0.00006$ \\
\hline $\mathrm{J} 2120-0058$ & 2120 & $25.9,-005827$ & 15 Sep. & $4 \times 900$ & 1.172 & $1{ }^{\prime \prime} 54$ & $0.01979 \pm 0.00004$ \\
\hline $\mathrm{J} 2150+0033$ & 2150 & 32. 0,003305 & 15 Sep. & $3 \times 800$ & 1.155 & 2.32 & $0.01508 \pm 0.00003$ \\
\hline G2155572-394614 & 2155 & 57. $9,-394614$ & 15 Sep. & $3 \times 900$ & 1.241 & $1 . " 90$ & $0.00740 \pm 0.00008$ \\
\hline J2227-0939 & 2227 & $30.7,-093954$ & 14 Sep. & $3 \times 800$ & 1.060 & $2 ! ' 14$ & $0.00528 \pm 0.00007$ \\
\hline PHL 293B & 2230 & $36.8,-000637$ & 14 Sep. & $4 \times 900$ & 1.230 & 1.35 & $0.00537 \pm 0.00004$ \\
\hline J2310-0109 No. 1 & 2310 & 42. $0,-010948$ & 16 Sep. & $3 \times 800$ & 1.471 & 1." 45 & $0.01254 \pm 0.00008$ \\
\hline J2310-0109 No. 2 & $23 \quad 10$ & 42. $0,-010948$ & 16 Sep. & $3 \times 800$ & 1.471 & 1." 45 & $0.01232 \pm 0.00009$ \\
\hline
\end{tabular}


N. G. Guseva et al.: The L-Z relation for a large sample of low-metallicity galaxies, Online Material p 2

Table 2. List of galaxies observed with the VLT ${ }^{a}$.

\begin{tabular}{|c|c|c|c|c|c|c|c|}
\hline Galaxy & & $\begin{array}{c}\text { RA, Dec. } \\
\text { (J2000) }\end{array}$ & Date & $\begin{array}{l}\text { Exp. time } \\
\text { (s) }\end{array}$ & Airmass & Seeing & Redshift \\
\hline \multirow[t]{2}{*}{ J0004+0025 No. 1} & $00^{\mathrm{h}} 04$ & ${ }^{\mathrm{n}} 21^{\mathrm{s}} 6, \quad 00^{\circ} 25^{\prime} 36^{\prime \prime}$ & 20.10 .2006 & $2 \times 750$ & 1.246 & $0 . ' 60$ & $0.01269 \pm 0.00004$ \\
\hline & & & & $2 \times 540$ & 1.181 & 0.37 & $0.01269 \pm 0.00004$ \\
\hline \multirow[t]{2}{*}{ J0004+0025 No. 2} & 0004 & $21.6, \quad 002536$ & 20.10 .2006 & $2 \times 750$ & 1.246 & $0 .{ }^{\prime} 60$ & $0.01266 \pm 0.00003$ \\
\hline & & & & $2 \times 540$ & 1.181 & $0 ! 37$ & $0.01266 \pm 0.00003$ \\
\hline \multirow[t]{2}{*}{ J0014-0044 No. 1} & 0014 & 28. 8, -00 4444 & 19.11.2006 & $2 \times 750$ & 1.158 & $1 . .22$ & $0.01361 \pm 0.00004$ \\
\hline & & & & $2 \times 540$ & 1.217 & 0.93 & $0.01361 \pm 0.00004$ \\
\hline \multirow[t]{2}{*}{ J0014-0044 No. 2} & 0014 & 28. 8, -00 4444 & 19.11.2006 & $2 \times 750$ & 1.158 & $1 . \prime 22$ & $0.01379 \pm 0.00002$ \\
\hline & & & & $2 \times 540$ & 1.217 & 0.93 & $0.01379 \pm 0.00002$ \\
\hline \multirow[t]{2}{*}{ J0202-0047 } & 0202 & 38. $0,-004744$ & 13.01 .2007 & $2 \times 750$ & 1.211 & $1 . \prime 85$ & $0.03371 \pm 0.00003$ \\
\hline & & & & $2 \times 540$ & 1.294 & 1.'63 & $0.03371 \pm 0.00003$ \\
\hline \multirow[t]{2}{*}{ J0301-0059 No. 1} & 0301 & 26. $3,-005926$ & 13.12.2006 & $2 \times 750$ & 1.140 & 1." 46 & $0.03841 \pm 0.00000$ \\
\hline & & & & $2 \times 540$ & 1.191 & 1.'38 & $0.03841 \pm 0.00000$ \\
\hline \multirow[t]{2}{*}{ J0301-0059 No. 2} & 0301 & 26. $3,-005926$ & 13.12 .2006 & $2 \times 750$ & 1.140 & 1."46 & $0.03822 \pm 0.00002$ \\
\hline & & & & $2 \times 540$ & 1.191 & 1.'38 & $0.03822 \pm 0.00002$ \\
\hline \multirow[t]{2}{*}{ J0301-0059 No. 3} & 0301 & 26. $3,-005926$ & 13.12 .2006 & $2 \times 750$ & 1.140 & 1."46 & $0.03807 \pm 0.00008$ \\
\hline & & & & $2 \times 540$ & 1.191 & 1.'38 & $0.03807 \pm 0.00008$ \\
\hline \multirow[t]{2}{*}{ J0315-0024 No. 1} & 0315 & $59.9,-002426$ & 26.11 .2006 & $2 \times 1500$ & 1.145 & 1.'32 & $0.02247 \pm 0.00005$ \\
\hline & & & & $2 \times 1080$ & 1.197 & $1 . \prime 08$ & $0.02247 \pm 0.00005$ \\
\hline \multirow[t]{2}{*}{ J0315-0024 No. 2} & 0315 & 59. 9, -00 2426 & 26.11 .2006 & $2 \times 1500$ & 1.145 & 1.32 & $0.02261 \pm 0.00006$ \\
\hline & & & & $2 \times 1080$ & 1.197 & $1 . \prime 08$ & $0.02261 \pm 0.00006$ \\
\hline \multirow[t]{2}{*}{ J0338+0013 (BG) } & 0338 & $\begin{array}{llll}12.2, & 00 & 13 & 13\end{array}$ & 25.11 .2006 & $2 \times 750$ & 1.207 & $0 ! 76$ & $0.39695 \pm 0.00000$ \\
\hline & & & & $2 \times 540$ & 1.284 & 0.70 & $0.39695 \pm 0.00000$ \\
\hline \multirow[t]{2}{*}{$\mathrm{J} 0338+0013$} & 0338 & $\begin{array}{llll}12.2, & 00 & 13 & 13\end{array}$ & 25.11 .2006 & $2 \times 750$ & 1.207 & $0 ! 76$ & $0.04266 \pm 0.00002$ \\
\hline & & & & $2 \times 540$ & 1.284 & $0 ! 70$ & $0.04266 \pm 0.00002$ \\
\hline \multirow[t]{2}{*}{ G0405204-364859 No. 1} & 0405 & 18. $6,-364849$ & 15.11 .2006 & $2 \times 750$ & 1.033 & 1."11 & $0.00281 \pm 0.00004$ \\
\hline & & & & $2 \times 540$ & 1.054 & $1 . .00$ & $0.00281 \pm 0.00004$ \\
\hline \multirow[t]{2}{*}{ G0405204-364859 No. 2} & 0405 & 18. $6,-364849$ & 15.11 .2006 & $2 \times 750$ & 1.033 & 1.11 & $0.00275 \pm 0.00003$ \\
\hline & & & & $2 \times 540$ & 1.054 & $1 . .00$ & $0.00275 \pm 0.00003$ \\
\hline \multirow[t]{2}{*}{ G0405204-364859 No. 3} & 0405 & 18. $6,-364849$ & 15.11.2006 & $2 \times 750$ & 1.033 & $1 " .11$ & $0.00276 \pm 0.00003$ \\
\hline & & & & $2 \times 540$ & 1.054 & $1 . \prime 00$ & $0.00276 \pm 0.00003$ \\
\hline \multirow[t]{2}{*}{ G0405204-364859 (BG) } & 0405 & 18. $6,-364849$ & 15.11 .2006 & $2 \times 750$ & 1.033 & $1 . \prime 11$ & $0.17236 \pm 0.00000$ \\
\hline & & & & $2 \times 540$ & 1.054 & $1 . \prime 00$ & $0.17236 \pm 0.00000$ \\
\hline \multirow[t]{2}{*}{ J0519+0007 } & 0519 & $02.7, \quad 0007 \quad 29$ & 16.11 .2006 & $2 \times 750$ & 1.220 & $1 . \prime 03$ & $0.04438 \pm 0.00002$ \\
\hline & & & & $2 \times 540$ & 1.166 & $1 . .27$ & $0.04438 \pm 0.00002$ \\
\hline J2104-0035 No. 1 & 2104 & $55.3,-003522$ & 12.10.2006 & $2 \times 750$ & 1.099 & 1.48 & $0.00469 \pm 0.00002$ \\
\hline & & & & $2 \times 540$ & 1.096 & 1." 40 & $0.00469 \pm 0.00002$ \\
\hline J2104-0035 No. 2 & 2104 & $55.3,-003522$ & 12.10.2006 & $2 \times 750$ & 1.099 & 1.'48 & $0.00469 \pm 0.00001$ \\
\hline & & & & $2 \times 540$ & 1.096 & 1." 40 & $0.00469 \pm 0.00001$ \\
\hline J2104-0035 No. 3 & 2104 & $55.3,-003522$ & 12.10 .2006 & $2 \times 750$ & 1.099 & 1.48 & $0.00471 \pm 0.00005$ \\
\hline & & & & $2 \times 540$ & 1.096 & 1." 40 & $0.00471 \pm 0.00005$ \\
\hline J2104-0035 No. 4 & 2104 & $55.3,-003522$ & 12.10.2006 & $2 \times 750$ & 1.099 & 1.48 & $0.00472 \pm 0.00003$ \\
\hline & & & & $2 \times 540$ & 1.096 & 1." 40 & $0.00472 \pm 0.00003$ \\
\hline J2302+0049 No. 1 & 2302 & 10. $0, \quad 004939$ & 12.10.2006 & $2 \times 750$ & 1.170 & 1.18 & $0.03312 \pm 0.00002$ \\
\hline & & & & $2 \times 540$ & 1.132 & $1 . ' 23$ & $0.03312 \pm 0.00002$ \\
\hline J2302+0049 No. 2 & 2302 & 10. $0, \quad 004939$ & 12.10 .2006 & $2 \times 750$ & 1.170 & 1.' 18 & $0.03311 \pm 0.00002$ \\
\hline & & & & $2 \times 540$ & 1.132 & 1.223 & $0.03311 \pm 0.00002$ \\
\hline $\mathrm{J} 2324-0006$ & 2324 & $21.3,-000629$ & 12.10 .2006 & $2 \times 750$ & 1.102 & 1.55 & $0.00896 \pm 0.00002$ \\
\hline & & & & $2 \times 540$ & 1.119 & $1 . .53$ & $0.00896 \pm 0.00002$ \\
\hline J2354-0005 No. 1 & 2354 & $37.3,-00 \quad 0502$ & 15.10 .2006 & $2 \times 1500$ & 1.502 & $0 ! 74$ & $0.00771 \pm 0.00003$ \\
\hline & & & & $2 \times 1080$ & 1.362 & 0.77 & $0.00771 \pm 0.00003$ \\
\hline J2354-0005 No. 2 & 2354 & 37. $3,-000502$ & 15.10 .2006 & $2 \times 1500$ & 1.502 & $0 ! 74$ & $0.00798 \pm 0.00004$ \\
\hline & & & & $2 \times 1080$ & 1.362 & $0 ! 77$ & $0.00798 \pm 0.00004$ \\
\hline $\mathrm{J} 2354-0005$ (BG1) & 2354 & $37.3,-000502$ & 15.10 .2006 & $2 \times 1500$ & 1.502 & $0 ! 74$ & $0.16534 \pm 0.00000$ \\
\hline & & & & $2 \times 1080$ & 1.362 & $0 ! 77$ & $0.16534 \pm 0.00000$ \\
\hline $\mathrm{J} 2354-0005$ (BG2) & 2354 & 37. $3,-000502$ & 15.10 .2006 & $2 \times 1500$ & 1.502 & 0.74 & $0.16520 \pm 0.00000$ \\
\hline & & & & $2 \times 1080$ & 1.362 & 0.77 & $0.16520 \pm 0.00000$ \\
\hline
\end{tabular}

${ }^{a}$ First line for each galaxy is related to the observation in the blue range and second line to the one in the red range. 
N. G. Guseva et al.: The L-Z relation for a large sample of low-metallicity galaxies, Online Material p 3

Table 3. Emission line intensities (3.6 m ESO observations).

\begin{tabular}{|c|c|c|c|c|c|c|c|c|}
\hline Ion & 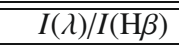 & $\overline{\overline{I(\lambda) / I(\mathrm{H} \beta)}}$ & $\overline{I I(\lambda) / I(\mathrm{H} \beta)}$ & $\overline{\bar{I} I(\lambda) / I(\mathrm{H} \beta)}$ & $\overline{I I(\lambda) / I(\mathrm{H} \beta)}$ & $\overline{I I(\lambda) / I(\mathrm{H} \beta)}$ & $\overline{I I(\lambda) / I(\mathrm{H} \beta)}$ & $\overline{\overline{I(\lambda) / I(\mathrm{H} \beta)}}$ \\
\hline & \multicolumn{8}{|c|}{ GALAXY } \\
\hline & J0015+0104 & J0016+0108 & J0029-0108 & J0029-0025 & J0057-0022 & J0107+0001 & J0109+0107 & $\begin{array}{r}\text { J0126-0038 } \\
\text { No. } 1 \\
\end{array}$ \\
\hline 3727 [O II] & $118.7 \pm 5.7$ & $221.2 \pm 7.8$ & $171.6 \pm 30.8$ & $130.3 \pm 7.8$ & $219.7 \pm 5.4$ & $162.3 \pm 13.6$ & $209.3 \pm 4.3$ & $238.1 \pm 4.1$ \\
\hline 3868 [Ne III] & $\ldots$ & $\ldots$ & $\ldots$ & $\ldots$ & $31.0 \pm 2.0$ & $\ldots$ & $30.1 \pm 1.0$ & $38.5 \pm 0.9$ \\
\hline $3889 \mathrm{He} \mathrm{I}+\mathrm{H} 8$ & $\ldots$ & $\ldots$ & $\ldots$ & $\ldots$ & $17.3 \pm 1.8$ & $\ldots$ & $20.6 \pm 1.6$ & $17.2 \pm 0.9$ \\
\hline $3968[\mathrm{Ne}$ III] + H7 & $\ldots$ & $\ldots$ & $\ldots$ & $\ldots$ & $16.1 \pm 1.9$ & $\ldots$ & $25.6 \pm 1.5$ & $22.2 \pm 0.9$ \\
\hline $4101 \mathrm{H} \delta$ & $\ldots$ & $\ldots$ & $\ldots$ & $\ldots$ & $24.9 \pm 1.9$ & $\ldots$ & $24.9 \pm 1.3$ & $24.8 \pm 0.9$ \\
\hline $4340 \mathrm{H} \gamma$ & $49.3 \pm 3.5$ & $47.0 \pm 3.4$ & $\ldots$ & $43.9 \pm 4.8$ & $47.2 \pm 1.9$ & $\ldots$ & $46.9 \pm 1.4$ & $46.9 \pm 1.0$ \\
\hline 4363 [O III] & $\ldots$ & $\ldots$ & $\ldots$ & $\ldots$ & $5.1 \pm 0.1$ & $\ldots$ & $3.7 \pm 0.4$ & $6.9 \pm 0.4$ \\
\hline $4471 \mathrm{He} \mathrm{I}$ & $\ldots$ & $\ldots$ & $\ldots$ & $\ldots$ & $\ldots$ & $\ldots$ & $2.6 \pm 0.4$ & $2.4 \pm 0.3$ \\
\hline 4658 [Fe III] & $\ldots$ & $\ldots$ & $\ldots$ & $\ldots$ & $\ldots$ & $\ldots$ & $\ldots$ & $1.5 \pm 0.3$ \\
\hline $4686 \mathrm{He}$ II & $\ldots$ & $\ldots$ & $\ldots$ & $\ldots$ & $\ldots$ & $\ldots$ & $\ldots$ & $1.7 \pm 0.3$ \\
\hline $4861 \mathrm{H} \beta$ & $100.0 \pm 4.9$ & $100.0 \pm 4.2$ & $100.0 \pm 15.0$ & $100.0 \pm 6.2$ & $100.0 \pm 2.6$ & $100.0 \pm 8.0$ & $100.0 \pm 2.1$ & $100.0 \pm 1.7$ \\
\hline 4959 [O III] & $20.1 \pm 2.0$ & $61.1 \pm 2.8$ & $48.1 \pm 8.8$ & $38.8 \pm 3.2$ & $76.1 \pm 2.0$ & $31.0 \pm 3.3$ & $102.5 \pm 2.1$ & $139.7 \pm 2.3$ \\
\hline 5007 [O III] & $57.8 \pm 2.8$ & $190.6 \pm 6.1$ & $144.3 \pm 22.7$ & $168.6 \pm 7.9$ & $219.4 \pm 4.8$ & $93.1 \pm 7.9$ & $305.1 \pm 5.6$ & $409.4 \pm 6.4$ \\
\hline$C(\mathrm{H} \beta)^{a}$ & 0.000 & 0.000 & 0.000 & 0.000 & 0.245 & 0.000 & 0.120 & 0.410 \\
\hline$F(\mathrm{H} \beta)^{b}$ & 2.77 & 4.66 & 1.19 & 2.16 & 12.99 & 2.88 & 18.18 & 75.47 \\
\hline $\mathrm{EW}(\mathrm{H} \beta)(\AA)$ & 57.5 & 20.4 & 6.8 & 53.8 & 44.6 & 87.7 & 50.1 & 48.7 \\
\hline \multirow[t]{4}{*}{$\operatorname{EW}(\mathrm{abs})(\AA)^{a}$} & 0.00 & 0.00 & 0.00 & 2.00 & 0.25 & 2.00 & 2.15 & 0.70 \\
\hline & \multicolumn{8}{|c|}{ GALAXY } \\
\hline & J0126-0038 & J0135-0023 & J0213-0002 & J0213-0002 & $\mathrm{J} 0216+0115$ & $\mathrm{~J} 0216+0115$ & 096632 & $\mathrm{~J} 0252+0017$ \\
\hline & No. 2 & & No. 1 & No. 2 & No. 1 & No. 2 & & \\
\hline 3727 [O II] & $224.9 \pm 6.3$ & $177.9 \pm 10.0$ & $182.2 \pm 9.8$ & $291.0 \pm 16.6$ & $225.2 \pm 8.7$ & $236.5 \pm 12.4$ & $306.5 \pm 6.9$ & $257.0 \pm 26.1$ \\
\hline 3868 [Ne III] & $\ldots$ & $\ldots$ & $\ldots$ & $\ldots$ & $\ldots$ & $\ldots$ & $8.4 \pm 0.9$ & $\ldots$ \\
\hline $4101 \mathrm{H} \delta$ & $24.8 \pm 2.2$ & $\ldots$ & $\ldots$ & $\ldots$ & $\ldots$ & $\ldots$ & $29.7 \pm 1.7$ & $\ldots$ \\
\hline $4340 \mathrm{H} \gamma$ & $44.8 \pm 2.2$ & $44.0 \pm 5.8$ & $47.1 \pm 5.0$ & $47.3 \pm 7.3$ & $49.3 \pm 3.9$ & $\ldots$ & $48.1 \pm 1.8$ & $\ldots$ \\
\hline $4861 \mathrm{H} \beta$ & $100.0 \pm 3.0$ & $100.0 \pm 6.7$ & $100.0 \pm 6.6$ & $100.0 \pm 7.6$ & $100.0 \pm 4.6$ & $100.0 \pm 10.0$ & $100.0 \pm 2.4$ & $100.0 \pm 10.0$ \\
\hline 4959 [O III] & $59.3 \pm 1.9$ & $43.5 \pm 3.5$ & $97.0 \pm 5.8$ & $115.6 \pm 7.2$ & $83.8 \pm 3.8$ & $42.1 \pm 3.9$ & $35.2 \pm 1.2$ & $71.7 \pm 8.5$ \\
\hline 5007 [O III] & $170.7 \pm 4.3$ & $155.9 \pm 7.9$ & $279.2 \pm 13.1$ & $332.9 \pm 16.7$ & $245.6 \pm 8.3$ & $126.9 \pm 6.7$ & $100.3 \pm 2.3$ & $270.5 \pm 24.1$ \\
\hline$C(\mathrm{H} \beta)^{a}$ & 0.000 & 0.000 & 0.000 & 0.000 & 0.000 & 0.000 & 0.000 & 0.000 \\
\hline$F(\mathrm{H} \beta)^{b}$ & 7.47 & 2.87 & 2.54 & 1.73 & 3.11 & 2.26 & 10.20 & 1.69 \\
\hline $\mathrm{EW}(\mathrm{H} \beta)(\AA)$ & 45.6 & 14.0 & 52.1 & 36.7 & 17.2 & 24.3 & 48.3 & 11.1 \\
\hline \multirow[t]{4}{*}{$\operatorname{EW}(\mathrm{abs})(\AA)^{a}$} & 2.00 & 2.00 & 2.00 & 0.00 & 0.00 & 0.00 & 2.00 & 2.00 \\
\hline & \multicolumn{8}{|c|}{ GALAXY } \\
\hline & $\mathrm{J} 0256+0036$ & J0303-0109 & J0303-0109 & J0341-0026 & J0341-0026 & J0341-0026 & G1815-6701 & G2052-6912 \\
\hline & & No. 1 & No. 2 & No. 1 & No. 2 & No. 3 & & No. 1 \\
\hline 3727 [O II] & $190.3 \pm 5.1$ & $137.9 \pm 4.5$ & $170.4 \pm 22.5$ & $227.5 \pm 13.6$ & $136.4 \pm 5.9$ & $87.5 \pm 5.0$ & $263.8 \pm 4.4$ & $137.6 \pm 2.1$ \\
\hline $3750 \mathrm{H} 12$ & $\ldots$ & $\ldots$ & $\ldots$ & $\ldots$ & $\ldots$ & $\ldots$ & $\ldots$ & $3.3 \pm 0.2$ \\
\hline 3771 H11 & $\ldots$ & $\ldots$ & $\ldots$ & $\ldots$ & $\ldots$ & $\ldots$ & $\ldots$ & $3.8 \pm 0.2$ \\
\hline 3798 H10 & $\ldots$ & $\ldots$ & $\ldots$ & $\ldots$ & $\ldots$ & $\ldots$ & $\ldots$ & $5.1 \pm 0.2$ \\
\hline 3835 H9 & $\ldots$ & $\ldots$ & $\ldots$ & $\ldots$ & $\ldots$ & $\ldots$ & $\ldots$ & $7.1 \pm 0.2$ \\
\hline 3868 [Ne III] & $31.7 \pm 1.7$ & $42.9 \pm 2.2$ & $\ldots$ & $\ldots$ & $\ldots$ & $\ldots$ & $52.3 \pm 1.1$ & $37.2 \pm 0.6$ \\
\hline $3889 \mathrm{He} \mathrm{I}+\mathrm{H} 8$ & $\ldots$ & $30.6 \pm 2.8$ & $\ldots$ & $\ldots$ & $\ldots$ & $\ldots$ & $17.5 \pm 0.8$ & $19.3 \pm 0.4$ \\
\hline $3968[\mathrm{Ne}$ III $]+\mathrm{H} 7$ & $\ldots$ & $36.4 \pm 3.0$ & $\ldots$ & $\ldots$ & $\ldots$ & $\ldots$ & $25.7 \pm 0.8$ & $27.2 \pm 0.5$ \\
\hline $4026 \mathrm{He} \mathrm{I}$ & $\ldots$ & $\ldots$ & $\ldots$ & $\ldots$ & $\ldots$ & $\ldots$ & $\ldots$ & $1.5 \pm 0.1$ \\
\hline 4068 [S II] & $\ldots$ & $\ldots$ & $\ldots$ & $\ldots$ & $\ldots$ & $\ldots$ & & $1.3 \pm 0.1$ \\
\hline $4101 \mathrm{H} \delta$ & $\ldots$ & $33.8 \pm 2.7$ & $\ldots$ & $\ldots$ & $\ldots$ & $\ldots$ & $23.7 \pm 0.8$ & $25.9 \pm 0.4$ \\
\hline $4340 \mathrm{H} \gamma$ & $47.8 \pm 2.2$ & $47.0 \pm 2.5$ & $\ldots$ & $\ldots$ & $45.4 \pm 4.4$ & $50.0 \pm 4.7$ & $46.9 \pm 1.0$ & $46.6 \pm 0.7$ \\
\hline 4363 [O III] & $\ldots$ & $8.2 \pm 1.0$ & $\ldots$ & $\ldots$ & $\ldots$ & $\ldots$ & $8.7 \pm 0.4$ & $4.1 \pm 0.1$ \\
\hline $4471 \mathrm{He} \mathrm{I}$ & $\ldots$ & $\ldots$ & $\ldots$ & $\ldots$ & $\ldots$ & $\ldots$ & $3.5 \pm 0.4$ & $3.9 \pm 0.1$ \\
\hline 4658 [Fe III] & $\ldots$ & $\ldots$ & $\ldots$ & $\ldots$ & $\ldots$ & $\ldots$ & $1.7 \pm 0.3$ & $0.3 \pm 0.1$ \\
\hline $4686 \mathrm{He}$ II & $\ldots$ & $\ldots$ & $\ldots$ & $\ldots$ & $\ldots$ & $\ldots$ & $1.6 \pm 0.4$ & $0.3 \pm 0.1$ \\
\hline $4711[\mathrm{Ar}$ IV $]+\mathrm{He}$ I & $\ldots$ & $\ldots$ & $\ldots$ & $\ldots$ & $\ldots$ & $\ldots$ & $\ldots$ & $0.7 \pm 0.1$ \\
\hline $4861 \mathrm{H} \beta$ & $100.0 \pm 3.0$ & $100.0 \pm 3.0$ & $100.0 \pm 13.0$ & $100.0 \pm 8.0$ & $100.0 \pm 4.7$ & $100.0 \pm 5.2$ & $100.0 \pm 1.7$ & $100.0 \pm 1.5$ \\
\hline $4921 \mathrm{He} \mathrm{I}$ & $\ldots$ & $\ldots$ & $\ldots$ & $18.9 \pm 2.8$ & $\ldots$ & $\ldots$ & $\ldots$ & $0.9 \pm 0.1$ \\
\hline 4959 [O III] & $110.1 \pm 3.0$ & $130.6 \pm 3.5$ & $26.6 \pm 5.1$ & $68.0 \pm 4.9$ & $77.0 \pm 3.5$ & $65.3 \pm 3.3$ & $171.5 \pm 2.7$ & $160.5 \pm 2.3$ \\
\hline 5007 [O III] & $316.2 \pm 7.3$ & $368.4 \pm 8.9$ & $79.9 \pm 11.6$ & $253.6 \pm 13.9$ & $243.1 \pm 8.4$ & $186.1 \pm 7.6$ & $508.6 \pm 7.9$ & $481.2 \pm 7.0$ \\
\hline
\end{tabular}


N. G. Guseva et al.: The L-Z relation for a large sample of low-metallicity galaxies, Online Material p 4

Table 3. continued.

\begin{tabular}{|c|c|c|c|c|c|c|c|c|}
\hline Ion & $\overline{I I(\lambda) / I(\mathrm{H} \beta)}$ & $\overline{I I(\lambda) / I(\mathrm{H} \beta)}$ & $\overline{I I(\lambda) / I(\mathrm{H} \beta)}$ & $\overline{I I(\lambda) / I(\mathrm{H} \beta)}$ & $\overline{I(\lambda) / I(\mathrm{H} \beta)}$ & $\overline{I I(\lambda) / I(\mathrm{H} \beta)}$ & $\overline{I I(\lambda) / I(\mathrm{H} \beta)}$ & $\overline{I(\lambda) / I(\mathrm{H} \beta)}$ \\
\hline$C(\mathrm{H} \beta)^{a}$ & 0.000 & 0.515 & 0.000 & 0.000 & 0.000 & 0.000 & 0.575 & 0.390 \\
\hline$F(\mathrm{H} \beta)^{b}$ & 9.25 & 11.86 & 1.71 & 4.49 & 4.49 & 3.54 & 66.43 & 448.40 \\
\hline $\mathrm{EW}(\mathrm{H} \beta)(\AA)$ & 26.2 & 95.0 & 21.2 & 27.5 & 85.2 & 145.8 & 35.9 & 221.4 \\
\hline \multirow[t]{4}{*}{$\operatorname{EW}(\mathrm{abs})(\AA)^{a}$} & 0.00 & 1.50 & 2.00 & 0.00 & 2.00 & 2.00 & 0.40 & 0.75 \\
\hline & & & & GALA & & & & \\
\hline & G2052-6912 & $\mathrm{J} 2053+0039$ & $\mathrm{~J} 2105+0032$ & $\mathrm{~J} 2105+0032$ & J2112-0016 & $\mathrm{J} 2112-0016$ & J2119-0732 & $\mathrm{J} 2120-0058$ \\
\hline & No. 2 & & No. 1 & No. 2 & No. 1 & No. 2 & & \\
\hline 3727 [O II] & $104.5 \pm 1.6$ & $85.8 \pm 8.2$ & $109.0 \pm 8.3$ & $187.0 \pm 10.8$ & $106.6 \pm 2.1$ & $107.6 \pm 4.6$ & $161.7 \pm 4.1$ & $252.5 \pm 5.3$ \\
\hline $3750 \mathrm{H} 12$ & $3.5 \pm 0.1$ & $\ldots$ & $\ldots$ & $\ldots$ & $\ldots$ & $\ldots$ & $\ldots$ & $\ldots$ \\
\hline $3771 \mathrm{H} 11$ & $4.1 \pm 0.1$ & $\ldots$ & $\ldots$ & $\ldots$ & $\ldots$ & $\ldots$ & $\ldots$ & $\ldots$ \\
\hline 3798 H10 & $5.2 \pm 0.2$ & $\ldots$ & $\ldots$ & $\ldots$ & $\ldots$ & $\ldots$ & $\ldots$ & $\ldots$ \\
\hline $3820 \mathrm{He} \mathrm{I}$ & $1.1 \pm 0.1$ & $\ldots$ & $\ldots$ & $\ldots$ & $\ldots$ & $\ldots$ & $\ldots$ & $\ldots$ \\
\hline 3835 H9 & $7.2 \pm 0.2$ & $\ldots$ & $\ldots$ & $\ldots$ & $\ldots$ & $\ldots$ & $\ldots$ & $\ldots$ \\
\hline 3868 [Ne III] & $47.9 \pm 0.7$ & $\ldots$ & $\ldots$ & $\ldots$ & $42.7 \pm 1.0$ & $25.7 \pm 2.0$ & $38.5 \pm 1.7$ & $39.9 \pm 1.4$ \\
\hline $3889 \mathrm{He} \mathrm{I}+\mathrm{H} 8$ & $18.1 \pm 0.3$ & $\ldots$ & $\ldots$ & $\ldots$ & $18.4 \pm 0.8$ & $18.0 \pm 3.1$ & $24.5 \pm 2.0$ & $22.5 \pm 1.7$ \\
\hline $3968[\mathrm{Ne} \mathrm{III}]+\mathrm{H} 7$ & $30.9 \pm 0.5$ & $\ldots$ & $\ldots$ & $\ldots$ & $27.9 \pm 1.0$ & $37.0 \pm 3.3$ & $\ldots$ & $20.4 \pm 1.7$ \\
\hline $4026 \mathrm{He} \mathrm{I}$ & $1.7 \pm 0.1$ & $\ldots$ & $\ldots$ & $\ldots$ & $\ldots$ & $\ldots$ & $\ldots$ & $\ldots$ \\
\hline 4068 [S II] & $1.0 \pm 0.1$ & $\ldots$ & $\ldots$ & $\ldots$ & $\ldots$ & $\ldots$ & $\ldots$ & $\ldots$ \\
\hline $4101 \mathrm{H} \delta$ & $25.9 \pm 0.4$ & $\ldots$ & $\ldots$ & $\ldots$ & $25.8 \pm 1.0$ & $41.1 \pm 2.4$ & $26.8 \pm 1.8$ & $27.5 \pm 1.5$ \\
\hline $4340 \mathrm{H} \gamma$ & $46.7 \pm 0.7$ & $\ldots$ & $\ldots$ & $\ldots$ & $48.7 \pm 1.1$ & $46.9 \pm 3.0$ & $46.7 \pm 1.8$ & $47.2 \pm 1.6$ \\
\hline 4363 [O III] & $6.7 \pm 0.1$ & $\ldots$ & $\ldots$ & $\ldots$ & $5.3 \pm 0.4$ & $5.5 \pm 0.9$ & $\ldots$ & $7.8 \pm 0.8$ \\
\hline $4387 \mathrm{He} \mathrm{I}$ & $0.6 \pm 0.1$ & $\ldots$ & $\ldots$ & $\ldots$ & $\ldots$ & $\ldots$ & $\ldots$ & $\ldots$ \\
\hline $4471 \mathrm{He} \mathrm{I}$ & $4.1 \pm 0.1$ & $\ldots$ & $\ldots$ & $\ldots$ & $3.7 \pm 0.3$ & $\ldots$ & $\ldots$ & $\ldots$ \\
\hline 4658 [Fe III] & $0.2 \pm 0.1$ & $\ldots$ & $\ldots$ & $\ldots$ & ... & $\ldots$ & $\ldots$ & $\ldots$ \\
\hline $4711[\mathrm{Ar}$ IV] $+\mathrm{He}$ I & $1.4 \pm 0.1$ & $\ldots$ & $\ldots$ & $\ldots$ & $\ldots$ & $\ldots$ & $\ldots$ & $\ldots$ \\
\hline 4740 [Ar IV] & $0.6 \pm 0.1$ & $\ldots$ & $\ldots$ & $\ldots$ & $\ldots$ & $\ldots$ & $\ldots$ & $\ldots$ \\
\hline $4861 \mathrm{H} \beta$ & $100.0 \pm 1.5$ & $100.0 \pm 9.0$ & $100.0 \pm 8.0$ & $100.0 \pm 6.0$ & $100.0 \pm 1.8$ & $100.0 \pm 3.5$ & $100.0 \pm 2.6$ & $100.0 \pm 2.2$ \\
\hline $4921 \mathrm{He}$ I & $1.1 \pm 0.1$ & & $\ldots$ & $\ldots$ & & $\ldots$ & & $\ldots$ \\
\hline 4959 [O III] & $207.9 \pm 3.0$ & $75.2 \pm 7.1$ & $71.2 \pm 5.3$ & $53.4 \pm 4.0$ & $189.9 \pm 3.2$ & $152.6 \pm 4.7$ & $137.3 \pm 3.1$ & $102.4 \pm 2.1$ \\
\hline 5007 [O III] & $623.9 \pm 9.0$ & $216.5 \pm 14.3$ & $263.8 \pm 14.4$ & $165.6 \pm 8.1$ & $565.0 \pm 9.1$ & $454.1 \pm 12.5$ & $413.1 \pm 8.6$ & $297.6 \pm 5.6$ \\
\hline$C(\mathrm{H} \beta)^{a}$ & 0.495 & 0.000 & 0.000 & 0.000 & 0.000 & 0.245 & 0.095 & 0.415 \\
\hline$F(\mathrm{H} \beta)^{b}$ & 766.20 & 1.58 & 2.57 & 2.36 & 49.75 & 7.37 & 16.82 & 15.15 \\
\hline $\operatorname{EW}(\mathrm{H} \beta)(\AA)$ & 395.9 & 24.4 & 18.2 & 121.7 & 95.2 & 176.1 & 52.8 & 40.1 \\
\hline \multirow[t]{3}{*}{$\operatorname{EW}(\mathrm{abs})(\AA)^{a}$} & 1.00 & 2.00 & 2.00 & 2.00 & 0.00 & 4.15 & 2.00 & 0.90 \\
\hline & \multicolumn{8}{|c|}{ GALAXY } \\
\hline & $\mathrm{J} 2150+0033$ & G2155-3946 & J2227-0939 & PHL 293B & J2310-0109 & J2310-0109 & & \\
\hline $3727[\mathrm{O} \mathrm{II}]$ & $190.0 \pm 7.3$ & $323.4 \pm 10.2$ & $197.0 \pm 7.0$ & $65.7 \pm 1.1$ & $\begin{array}{r}\text { No. } 1 \\
128.0 \pm 4.0\end{array}$ & $\begin{array}{r}\text { No. } 2 \\
177.4+6.0\end{array}$ & & \\
\hline $3750 \mathrm{H} 12$ & $\ldots$ & $\ldots$ & $\ldots$ & $5.0 \pm 0.4$ & $\begin{array}{c}\ldots \\
\ldots\end{array}$ & $\ldots$ & & \\
\hline 3771 H11 & $\ldots$ & $\ldots$ & $\ldots$ & $6.4 \pm 0.4$ & $\ldots$ & $\ldots$ & & \\
\hline $3798 \mathrm{H} 10$ & $\ldots$ & $\ldots$ & $\ldots$ & $8.2 \pm 0.4$ & $\ldots$ & $\ldots$ & & \\
\hline 3835 H9 & $\ldots$ & $\ldots$ & $\ldots$ & $8.4 \pm 0.4$ & $\ldots$ & $\ldots$ & & \\
\hline 3868 [Ne III] & $\ldots$ & $16.6 \pm 1.9$ & $35.0 \pm 3.0$ & $41.0 \pm 0.7$ & $20.9 \pm 1.4$ & $27.8 \pm 1.9$ & & \\
\hline $3889 \mathrm{He} \mathrm{I}+\mathrm{H} 8$ & $\ldots$ & $\ldots$ & $23.4 \pm 7.2$ & $21.4 \pm 0.5$ & $\ldots$ & $\ldots$ & & \\
\hline $3968[\mathrm{Ne} \mathrm{III]}+\mathrm{H} 7$ & $\ldots$ & $\ldots$ & $26.9 \pm 4.9$ & $28.9 \pm 0.6$ & $\ldots$ & $\ldots$ & & \\
\hline $4101 \mathrm{H} \delta$ & $\ldots$ & $22.8 \pm 4.6$ & $28.5 \pm 4.4$ & $25.7 \pm 0.5$ & $\ldots$ & $\ldots$ & & \\
\hline $4340 \mathrm{H} \gamma$ & $39.9 \pm 3.4$ & $47.1 \pm 3.0$ & $47.3 \pm 3.4$ & $47.4 \pm 0.8$ & $38.3 \pm 2.7$ & $41.5 \pm 3.4$ & & \\
\hline 4363 [O III] & $7.3 \pm 1.3$ & $\ldots$ & $\ldots$ & $12.3 \pm 0.3$ & $\ldots$ & $8.7 \pm 1.3$ & & \\
\hline $4471 \mathrm{He} \mathrm{I}$ & $\ldots$ & $\ldots$ & $\ldots$ & $3.5 \pm 0.2$ & $\ldots$ & $\ldots$ & & \\
\hline 4686 He II & $\ldots$ & $\ldots$ & $\ldots$ & $1.4 \pm 0.1$ & $\ldots$ & $\ldots$ & & \\
\hline $4711[\mathrm{Ar}$ IV] $+\mathrm{He}$ I & $\ldots$ & $\ldots$ & $\ldots$ & $1.8 \pm 0.1$ & $\ldots$ & $\ldots$ & & \\
\hline 4740 [Ar IV] & $\ldots$ & $\ldots$ & $\ldots$ & $1.1 \pm 0.1$ & & $\ldots$ & & \\
\hline $4861 \mathrm{H} \beta$ & $100.0 \pm 4.6$ & $100.0 \pm 3.8$ & $100.0 \pm 3.9$ & $100.0 \pm 1.5$ & $100.0 \pm 3.3$ & $100.0 \pm 3.8$ & & \\
\hline 4959 [O III] & $86.9 \pm 3.5$ & $59.3 \pm 2.4$ & $128.2 \pm 4.3$ & $157.1 \pm 2.4$ & $122.1 \pm 3.4$ & $115.5 \pm 3.7$ & & \\
\hline 5007 [O III] & $255.9 \pm 8.4$ & $170.9 \pm 5.1$ & $373.0 \pm 10.9$ & $466.7 \pm 7.0$ & $369.4 \pm 9.0$ & $332.8 \pm 9.2$ & & \\
\hline$C(\mathrm{H} \beta)^{a}$ & 0.000 & 0.000 & 0.000 & 0.305 & 0.000 & 0.000 & & \\
\hline$F(\mathrm{H} \beta)^{b}$ & 4.59 & 10.53 & 21.39 & 107.50 & 14.00 & 9.26 & & \\
\hline $\mathrm{EW}(\mathrm{H} \beta)(\AA)$ & 30.0 & 13.8 & 20.3 & 117.2 & 26.1 & 30.5 & & \\
\hline $\mathrm{EW}(\mathrm{abs})(\AA)^{a}$ & 2.00 & 2.00 & 2.00 & 1.50 & 2.00 & 2.00 & & \\
\hline
\end{tabular}

${ }^{a}$ Zero value is assumed if a negative value is derived. ${ }^{b}$ In units of $10^{-16} \mathrm{erg} \mathrm{s}^{-1} \mathrm{~cm}^{-2}$. 
N. G. Guseva et al.: The L-Z relation for a large sample of low-metallicity galaxies, Online Material p 5

Table 4. Emission line intensities (VLT observations).

\begin{tabular}{|c|c|c|c|c|c|c|c|c|}
\hline$\overline{\text { Ion }}$ & $\overline{I(\lambda) / I(\mathrm{H} \beta)}$ & $\overline{I(\lambda) / I(\mathrm{H} \beta)}$ & $\overline{I(\lambda) / I(\mathrm{H} \beta)}$ & $\overline{I I(\lambda) / I(\mathrm{H} \beta)}$ & $\overline{I I(\lambda) / I(\mathrm{H} \beta)}$ & $\overline{I I(\lambda) / I(\mathrm{H} \beta)}$ & $\overline{I(\lambda) / I(\mathrm{H} \beta)}$ & $\overline{I(\lambda) / I(\mathrm{H} \beta)}$ \\
\hline & \multicolumn{8}{|c|}{ GALAXY } \\
\hline & J0004+0025 & J0004+0025 & J0014-0044 & J0014-0044 & J0202-0047 & J0301-0059 & J0301-0059 & J0301-0059 \\
\hline & No. 1 & No. 2 & No. 1 & No. 2 & & No. 1 & No. 2 & No. 3 \\
\hline 3727 [O II] & $214.05 \pm 11.27$ & $253.26 \pm 13.99$ & $99.16 \pm 1.73$ & $298.77 \pm 9.30$ & $191.81 \pm 3.50$ & $331.24 \pm 6.29$ & $304.43 \pm 6.86$ & $330.51 \pm 21.26$ \\
\hline $3750 \mathrm{H} 12$ & $\ldots$ & $\ldots$ & $4.57 \pm 0.56$ & $\ldots$ & $\ldots$ & $\ldots$ & $\ldots$ & $\ldots$ \\
\hline 3771 H11 & $\ldots$ & $\ldots$ & $5.06 \pm 0.52$ & $\ldots$ & $4.22 \pm 0.66$ & $\ldots$ & $\ldots$ & $\ldots$ \\
\hline 3798 H10 & $\ldots$ & $\ldots$ & $7.06 \pm 0.52$ & $\ldots$ & $4.83 \pm 0.67$ & $\ldots$ & $\ldots$ & $\ldots$ \\
\hline 3835 H9 & $\ldots$ & $\ldots$ & $8.66 \pm 0.46$ & $\ldots$ & $5.85 \pm 0.58$ & $\ldots$ & $\ldots$ & $\ldots$ \\
\hline 3868 [Ne III] & $\cdots$ & $33.25 \pm 4.96$ & $50.11 \pm 0.85$ & $\cdots$ & $41.34 \pm 0.92$ & $24.06 \pm 0.98$ & $23.23 \pm 1.72$ & $\cdots$ \\
\hline $3889 \mathrm{He} \mathrm{I}+\mathrm{H} 8$ & $\ldots$ & $\ldots$ & $21.72 \pm 0.55$ & $\ldots$ & $22.44 \pm 0.72$ & $22.59 \pm 1.80$ & $\ldots$ & $\ldots$ \\
\hline $3968[\mathrm{Ne}$ III $]+\mathrm{H} 7$ & $\ldots$ & $\ldots$ & $32.07 \pm 0.64$ & $\ldots$ & $28.21 \pm 0.78$ & $25.37 \pm 1.37$ & $\ldots$ & $\ldots$ \\
\hline $4026 \mathrm{He} \mathrm{I}$ & $\cdots$ & $\cdots$ & $1.10 \pm 0.11$ & $\cdots$ & $\ldots$ & $\ldots$ & $\begin{array}{l}\cdots \\
\ldots\end{array}$ & $\ldots$ \\
\hline $4101 \mathrm{H} \delta$ & $\ldots$ & $\ldots$ & $25.84 \pm 0.51$ & $28.41 \pm 2.21$ & $25.71 \pm 0.70$ & $28.75 \pm 1.16$ & $28.14 \pm 2.37$ & $\ldots$ \\
\hline $4340 \mathrm{H} \gamma$ & $47.25 \pm 3.35$ & $47.74 \pm 3.21$ & $46.79 \pm 0.77$ & $51.88 \pm 1.98$ & $48.61 \pm 0.93$ & $46.88 \pm 1.15$ & $45.15 \pm 1.68$ & $\cdots$ \\
\hline 4363 [O III] & $\ldots$ & $\ldots$ & $8.87 \pm 0.18$ & $\ldots$ & $8.97 \pm 0.37$ & $3.46 \pm 0.42$ & $4.43 \pm 0.92$ & $\cdots$ \\
\hline $4387 \mathrm{He} \mathrm{I}$ & $\ldots$ & $\ldots$ & $0.57 \pm 0.08$ & $\ldots$ & $\ldots$ & $\ldots$ & $\ldots$ & $\ldots$ \\
\hline $4471 \mathrm{He} \mathrm{I}$ & $\ldots$ & $\ldots$ & $3.83 \pm 0.12$ & $\ldots$ & $3.49 \pm 0.26$ & $\ldots$ & $\ldots$ & $\ldots$ \\
\hline $4711[\mathrm{Ar} \mathrm{IV}]+\mathrm{He} \mathrm{I}$ & $\ldots$ & $\ldots$ & $1.08 \pm 0.09$ & $\ldots$ & $\ldots$ & $\ldots$ & $\ldots$ & $\ldots$ \\
\hline $4740[\mathrm{Ar} \mathrm{IV}]$ & $\ldots$ & $\ldots$ & $0.84 \pm 0.07$ & $\ldots$ & $\ldots$ & $\ldots$ & $\ldots$ & $\ldots$ \\
\hline $4861 \mathrm{H} \beta$ & $100.00 \pm 3.77$ & $100.00 \pm 3.98$ & $100.00 \pm 1.49$ & $100.00 \pm 2.52$ & $100.00 \pm 1.60$ & $100.00 \pm 1.81$ & $100.00 \pm 2.17$ & $100.00 \pm 17.01$ \\
\hline $4921 \mathrm{He}$ I & $\ldots$ & $\ldots$ & $0.94 \pm 0.06$ & $\ldots$ & $\ldots$ & $\ldots$ & $\ldots$ & $\ldots$ \\
\hline 4959 [O III] & $37.84 \pm 1.98$ & $62.06 \pm 2.64$ & $207.93 \pm 3.04$ & $59.47 \pm 1.64$ & $161.71 \pm 2.50$ & $91.37 \pm 1.62$ & $90.51 \pm 1.93$ & $53.80 \pm 4.75$ \\
\hline 4988 [Fe III] & $\ldots$ & $\ldots$ & $0.44 \pm 0.01$ & $\ldots$ & $\ldots$ & $\ldots$ & $\ldots$ & $\ldots$ \\
\hline 5007 [O III] & $108.99 \pm 3.45$ & $183.91 \pm 5.64$ & $621.54 \pm 9.04$ & $175.13 \pm 3.94$ & $495.48 \pm 7.53$ & $275.10 \pm 4.60$ & $267.82 \pm 5.17$ & $154.51 \pm 8.64$ \\
\hline $5015 \mathrm{He} \mathrm{I}$ & $\ldots$ & $\ldots$ & $0.90 \pm 0.03$ & $\ldots$ & $2.20 \pm 0.15$ & $\ldots$ & $\ldots$ & $\ldots$ \\
\hline 5518 [Cl III] & $\ldots$ & $\ldots$ & $0.38 \pm 0.06$ & $\ldots$ & $\ldots$ & $\ldots$ & $\ldots$ & $\ldots$ \\
\hline 5538 [Cl III] & $\ldots$ & $\ldots$ & $0.61 \pm 0.10$ & $\ldots$ & $\ldots$ & $\ldots$ & $\ldots$ & $\ldots$ \\
\hline $5876 \mathrm{He}$ I & $\ldots$ & $\ldots$ & $10.92 \pm 0.19$ & $13.81 \pm 0.29$ & $10.96 \pm 0.27$ & $9.94 \pm 0.61$ & $11.70 \pm 0.82$ & $\ldots$ \\
\hline $6300[\mathrm{O} \mathrm{I}]$ & $\ldots$ & $\ldots$ & $1.92 \pm 0.06$ & $\ldots$ & $3.11 \pm 0.13$ & $9.14 \pm 0.52$ & $9.57 \pm 0.60$ & $\ldots$ \\
\hline 6312 [S III] & $\ldots$ & $\ldots$ & $1.73 \pm 0.06$ & $\ldots$ & $1.74 \pm 0.11$ & $0.97 \pm 0.02$ & $\ldots$ & $\ldots$ \\
\hline $6363[\mathrm{O} \mathrm{I}]$ & $\ldots$ & $\cdots$ & $0.70 \pm 0.05$ & $\cdots$ & $1.42 \pm 0.02$ & $2.73 \pm 0.40$ & $2.72 \pm 0.05$ & $\cdots$ \\
\hline 6548 [N II] & $\ldots$ & $\ldots$ & $2.16 \pm 0.06$ & $10.39 \pm 0.88$ & $3.86 \pm 0.13$ & $6.66 \pm 0.44$ & $7.77 \pm 0.53$ & $\ldots$ \\
\hline $6563 \mathrm{H} \alpha$ & $269.07 \pm 8.14$ & $266.58 \pm 8.43$ & $283.02 \pm 4.47$ & $288.13 \pm 6.59$ & $281.45 \pm 4.63$ & $284.02 \pm 5.14$ & $281.73 \pm 5.83$ & $288.79 \pm 19.92$ \\
\hline 6583 [N II] & $15.55 \pm 1.51$ & $12.75 \pm 1.07$ & $5.73 \pm 0.11$ & $22.66 \pm 0.99$ & $9.59 \pm 0.21$ & $20.47 \pm 0.54$ & $26.57 \pm 0.81$ & $33.85 \pm 3.43$ \\
\hline $6678 \mathrm{He} \mathrm{I}$ & $\ldots$ & $\ldots$ & $3.05 \pm 0.07$ & $\ldots$ & $2.69 \pm 0.11$ & $1.83 \pm 0.35$ & $\ldots$ & $\ldots$ \\
\hline 6717 [S II] & $32.18 \pm 1.51$ & $27.90 \pm 1.41$ & $8.04 \pm 0.14$ & $31.47 \pm 1.01$ & $16.63 \pm 0.32$ & $42.25 \pm 0.88$ & $47.03 \pm 1.11$ & $63.87 \pm 4.85$ \\
\hline 6731 [S II] & $25.79 \pm 1.51$ & $22.66 \pm 1.32$ & $5.78 \pm 0.11$ & $24.87 \pm 0.91$ & $12.38 \pm 0.26$ & $29.45 \pm 0.68$ & $32.66 \pm 0.86$ & $44.23 \pm 4.15$ \\
\hline $7065 \mathrm{He} \mathrm{I}$ & $\ldots$ & $\ldots$ & $2.45 \pm 0.06$ & $\ldots$ & $1.66 \pm 0.09$ & $1.58 \pm 0.26$ & $\ldots$ & $\ldots$ \\
\hline 7136 [Ar III] & $\ldots$ & $\ldots$ & $6.61 \pm 0.12$ & $\ldots$ & $8.26 \pm 0.19$ & $6.60 \pm 0.41$ & $7.65 \pm 0.41$ & $\ldots$ \\
\hline $7281 \mathrm{He}$ I & $\ldots$ & $\ldots$ & $0.51 \pm 0.03$ & $\ldots$ & $0.92 \pm 0.08$ & $\ldots$ & $\ldots$ & $\ldots$ \\
\hline 7320 [O II] & $\ldots$ & $\ldots$ & $1.51 \pm 0.05$ & $\ldots$ & $2.16 \pm 0.09$ & $\ldots$ & $\ldots$ & $\ldots$ \\
\hline 7330 [O II] & $\ldots$ & $\ldots$ & $1.36 \pm 0.05$ & $\ldots$ & $1.85 \pm 0.07$ & $\ldots$ & $\ldots$ & $\ldots$ \\
\hline$C(\mathrm{H} \beta)^{a}$ & 0.000 & 0.000 & 0.210 & 0.030 & 0.305 & 0.215 & 0.185 & 0.110 \\
\hline$F(\mathrm{H} \beta)^{b}$ & 0.76 & 0.80 & 20.90 & 0.81 & 5.47 & 5.40 & $\begin{array}{r}3.59 \\
\end{array}$ & 0.38 \\
\hline $\mathrm{EW}(\mathrm{H} \beta)(\AA)$ & 22.5 & 21.4 & 275.7 & 74.2 & 132.6 & 28.3 & 13.6 & 8.0 \\
\hline \multirow[t]{4}{*}{ EW $(a b s)(\AA)$} & 2.00 & 2.00 & 2.20 & 2.00 & 0.50 & 1.95 & 1.75 & 2.00 \\
\hline & \multicolumn{8}{|c|}{ GALAXY } \\
\hline & J0315-0024 & J0315-0024 & $\mathrm{J} 0338+0013$ & G0405-3648 & G0405-3648 & G0405-3648 & J0519+0007 & $\mathrm{J} 2104-0035$ \\
\hline & No. 1 & No. 2 & & No. 1 & No. 2 & No. 3 & & No. 1 \\
\hline 3727 [O II] & $174.28 \pm 5.19$ & $190.40 \pm 9.12$ & $60.18 \pm 1.06$ & $129.50 \pm 5.31$ & $160.45 \pm 3.75$ & $154.37 \pm 3.90$ & $27.98 \pm 0.47$ & $27.37 \pm 0.97$ \\
\hline $3750 \mathrm{H} 12$ & $\ldots$ & $\ldots$ & $3.59 \pm 0.30$ & $\ldots$ & $\ldots$ & $\ldots$ & $3.13 \pm 0.18$ & $4.11 \pm 0.75$ \\
\hline 3771 H11 & $\ldots$ & $\ldots$ & $3.80 \pm 0.29$ & $\ldots$ & $\ldots$ & $\ldots$ & $4.07 \pm 0.18$ & $5.22 \pm 0.73$ \\
\hline $3798 \mathrm{H} 10$ & $\ldots$ & $\ldots$ & $6.86 \pm 0.30$ & $\ldots$ & $\ldots$ & $\ldots$ & $5.75 \pm 0.18$ & $6.80 \pm 0.66$ \\
\hline $3820 \mathrm{He} \mathrm{I}$ & $\ldots$ & $\ldots$ & $2.00 \pm 0.18$ & $\ldots$ & $\ldots$ & $\ldots$ & $0.74 \pm 0.09$ & $\ldots$ \\
\hline 3835 H9 & $\ldots$ & $\ldots$ & $8.51 \pm 0.28$ & $\ldots$ & $\ldots$ & $\ldots$ & $7.26 \pm 0.19$ & $8.01 \pm 0.61$ \\
\hline 3868 [Ne III] & $8.88 \pm 1.88$ & $\ldots$ & $41.54 \pm 0.71$ & $9.66 \pm 1.46$ & $11.59 \pm 1.16$ & $14.90 \pm 1.09$ & $35.86 \pm 0.56$ & $24.32 \pm 0.53$ \\
\hline $3889 \mathrm{He} \mathrm{I}+\mathrm{H} 8$ & $20.76 \pm 2.11$ & $\cdots$ & $18.64 \pm 0.41$ & $18.38 \pm 3.37$ & $18.70 \pm 1.09$ & $20.05 \pm 1.24$ & $17.21 \pm 0.31$ & $22.61 \pm 0.66$ \\
\hline $3968[\mathrm{Ne}$ III $]+\mathrm{H} 7$ & $16.88 \pm 1.83$ & $\ldots$ & $28.87 \pm 0.53$ & $17.38 \pm 2.88$ & $15.71 \pm 1.02$ & $20.71 \pm 1.21$ & $26.96 \pm 0.44$ & $23.31 \pm 0.65$ \\
\hline $4026 \mathrm{He} \mathrm{I}$ & $\ldots$ & $\ldots$ & $2.82 \pm 0.17$ & $\ldots$ & $\ldots$ & $\ldots$ & $1.55 \pm 0.08$ & $2.12 \pm 0.18$ \\
\hline 4068 [S II] & $\ldots$ & $\ldots$ & $1.16 \pm 0.02$ & $\ldots$ & $\ldots$ & $\ldots$ & $0.65 \pm 0.06$ & $\ldots$ \\
\hline $4101 \mathrm{H} \delta$ & $21.87 \pm 1.58$ & $\ldots$ & $26.20 \pm 0.47$ & $25.79 \pm 1.85$ & $21.62 \pm 0.82$ & $24.52 \pm 0.99$ & $25.67 \pm 0.41$ & $25.83 \pm 0.63$ \\
\hline $4227[\mathrm{Fe} \mathrm{v}]$ & $\ldots$ & $\ldots$ & $\ldots$ & $\ldots$ & $\ldots$ & $\ldots$ & $0.86 \pm 0.14$ & $\ldots$ \\
\hline $4340 \mathrm{H} \gamma$ & $49.15 \pm 1.56$ & $50.73 \pm 4.71$ & $48.06 \pm 0.76$ & $47.97 \pm 1.55$ & $45.48 \pm 1.00$ & $49.12 \pm 1.13$ & $47.62 \pm 0.71$ & $48.28 \pm 0.82$ \\
\hline 4363 [O III] & $2.04 \pm 0.74$ & $\ldots$ & $14.66 \pm 0.27$ & $4.02 \pm 0.76$ & $4.03 \pm 0.36$ & $5.12 \pm 0.50$ & $15.04 \pm 0.24$ & $9.87 \pm 0.20$ \\
\hline $4387 \mathrm{He} \mathrm{I}$ & $\ldots$ & $\cdots$ & $\ldots$ & ... & $\ldots$ & $\ldots$ & $0.37 \pm 0.05$ & $\ldots$ \\
\hline $4471 \mathrm{He}$ I & $\cdots$ & $\begin{array}{l}\cdots \\
\cdots\end{array}$ & $3.70 \pm 0.13$ & $2.18 \pm 0.72$ & $3.54 \pm 0.43$ & $2.63 \pm 0.40$ & $3.60 \pm 0.08$ & $3.65 \pm 0.13$ \\
\hline 4658 [Fe III] & $\ldots$ & $\ldots$ & $0.49 \pm 0.06$ & $\ldots$ & $\ldots$ & $\ldots$ & $0.49 \pm 0.04$ & $\ldots$ \\
\hline $4686 \mathrm{He}$ II & $\cdots$ & $\cdots$ & $1.15 \pm 0.08$ & $\begin{array}{l}\cdots \\
\ldots\end{array}$ & $\cdots$ & $\cdots$ & $1.52 \pm 0.05$ & $0.65 \pm 0.10$ \\
\hline 4711 [Ar IV] + He I & $\ldots$ & $\ldots$ & $2.35 \pm 0.09$ & $\ldots$ & $\ldots$ & $\ldots$ & $2.33 \pm 0.06$ & $1.52 \pm 0.09$ \\
\hline 4740 [Ar IV] & $\ldots$ & $\ldots$ & $1.40 \pm 0.07$ & $\ldots$ & $\ldots$ & $\ldots$ & $1.51 \pm 0.06$ & $0.74 \pm 0.10$ \\
\hline $4861 \mathrm{H} \beta$ & $100.00 \pm 1.95$ & $100.00 \pm 4.69$ & $100.00 \pm 1.48$ & $100.00 \pm 2.03$ & $100.00 \pm 1.70$ & $100.00 \pm 1.77$ & $100.00 \pm 1.44$ & $100.00 \pm 1.50$ \\
\hline $4921 \mathrm{He}$ I & $\ldots$ & $\ldots$ & $1.27 \pm 0.08$ & $\ldots$ & $\ldots$ & $\ldots$ & $0.98 \pm 0.05$ & $1.11 \pm 0.08$ \\
\hline 4959 [O III] & $53.68 \pm 1.19$ & $51.95 \pm 2.12$ & $176.71 \pm 2.58$ & $70.62 \pm 1.51$ & $48.62 \pm 0.91$ & $45.69 \pm 0.93$ & $148.33 \pm 2.13$ & $96.10 \pm 1.41$ \\
\hline 4988 [Fe III] & $\ldots$ & $\ldots$ & $0.54 \pm 0.05$ & $\ldots$ & $1.96 \pm 0.31$ & $\ldots$ & $0.65 \pm 0.04$ & $0.75 \pm 0.13$ \\
\hline $5007[\mathrm{O} \mathrm{III]}$ & $162.82 \pm 2.95$ & $151.44 \pm 4.43$ & $530.20 \pm 7.71$ & $206.51 \pm 3.80$ & $140.34 \pm 2.29$ & $137.62 \pm 2.33$ & $439.62 \pm 6.30$ & $288.91 \pm 4.20$ \\
\hline $5015 \mathrm{He} \mathrm{I}$ & $\ldots$ & $\ldots$ & $2.59 \pm 0.08$ & $\ldots$ & $\ldots$ & $\ldots$ & $0.97 \pm 0.04$ & $1.64 \pm 0.18$ \\
\hline 5755 [N II] & $\ldots$ & $\ldots$ & $\ldots$ & $\ldots$ & $\ldots$ & $\ldots$ & $0.15 \pm 0.02$ & $\ldots$ \\
\hline $5876 \mathrm{He} \mathrm{I}$ & $9.03 \pm 0.55$ & $\ldots$ & $11.14 \pm 0.21$ & $8.41 \pm 0.58$ & $9.77 \pm 0.48$ & $5.72 \pm 0.29$ & $11.60 \pm 0.18$ & $9.38 \pm 0.19$ \\
\hline $6300[\mathrm{O} \mathrm{I}]$ & $\ldots$ & $\begin{array}{l}\cdots \\
\ldots\end{array}$ & $1.72 \pm 0.07$ & $\ldots$ & $4.36 \pm 0.36$ & $2.75 \pm 0.25$ & $1.07 \pm 0.03$ & $0.49 \pm 0.06$ \\
\hline
\end{tabular}


N. G. Guseva et al.: The L-Z relation for a large sample of low-metallicity galaxies, Online Material p 6

Table 4. continued.

\begin{tabular}{|c|c|c|c|c|c|c|c|c|}
\hline 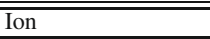 & $\overline{I I(\lambda) / I(\mathrm{H} \beta)}$ & 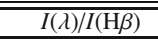 & $\overline{I I(\lambda) / I(\mathrm{H} \beta)}$ & $\overline{I I(\lambda) / I(\mathrm{H} \beta)}$ & $\overline{\overline{I(\lambda) / I(\mathrm{H} \beta)}}$ & $\overline{\overline{I(\lambda) / I(\mathrm{H} \beta)}}$ & $\overline{I I(\lambda) / I(\mathrm{H} \beta)}$ & $\overline{\overline{I(\lambda) / I(\mathrm{H} \beta)}}$ \\
\hline 6312 [S III] & $\ldots$ & $\ldots$ & $1.01 \pm 0.07$ & $\ldots$ & $\ldots$ & $\ldots$ & $0.78 \pm 0.02$ & $0.66 \pm 0.06$ \\
\hline $6363[\mathrm{O} \mathrm{I}]$ & $\ldots$ & $\ldots$ & $0.53 \pm 0.05$ & $\ldots$ & $\ldots$ & $\ldots$ & $0.37 \pm 0.02$ & $\ldots$ \\
\hline 6548 [N II] & $\ldots$ & $\ldots$ & $\ldots$ & $\ldots$ & $\ldots$ & $\ldots$ & $1.17 \pm 0.03$ & $\ldots$ \\
\hline $6563 \mathrm{H} \alpha$ & $283.73 \pm 5.33$ & $288.03 \pm 8.76$ & $277.02 \pm 4.37$ & $279.40 \pm 5.45$ & $275.30 \pm 4.73$ & $272.50 \pm 4.83$ & $274.85 \pm 4.27$ & $274.51 \pm 4.32$ \\
\hline 6583 [N II] & $6.88 \pm 0.30$ & $11.83 \pm 1.44$ & $1.64 \pm 0.06$ & $5.41 \pm 0.37$ & $6.48 \pm 0.31$ & $5.14 \pm 0.23$ & $3.10 \pm 0.05$ & $0.93 \pm 0.06$ \\
\hline $6678 \mathrm{He} \mathrm{I}$ & $1.89 \pm 0.04$ & $\ldots$ & $2.69 \pm 0.08$ & $\ldots$ & $2.31 \pm 0.24$ & $2.64 \pm 0.22$ & $2.95 \pm 0.05$ & $2.50 \pm 0.07$ \\
\hline 6717 [S II] & $8.51 \pm 0.36$ & $13.97 \pm 1.68$ & $4.65 \pm 0.10$ & $13.04 \pm 0.55$ & $16.04 \pm 0.40$ & $14.92 \pm 0.39$ & $2.36 \pm 0.04$ & $2.10 \pm 0.06$ \\
\hline 6731 [S II] & $9.99 \pm 0.33$ & $9.02 \pm 0.97$ & $3.55 \pm 0.09$ & $8.37 \pm 0.42$ & $12.20 \pm 0.35$ & $9.95 \pm 0.30$ & $2.19 \pm 0.04$ & $1.57 \pm 0.06$ \\
\hline $7065 \mathrm{He}$ I & $\ldots$ & $\ldots$ & $4.94 \pm 0.10$ & $2.53 \pm 0.33$ & $1.35 \pm 0.19$ & $1.90 \pm 0.19$ & $6.54 \pm 0.11$ & $2.30 \pm 0.06$ \\
\hline 7136 [Ar III] & $\ldots$ & $\ldots$ & $3.14 \pm 0.08$ & $3.69 \pm 0.30$ & $2.82 \pm 0.20$ & $2.33 \pm 0.16$ & $2.36 \pm 0.04$ & $1.25 \pm 0.05$ \\
\hline $7281 \mathrm{He} \mathrm{I}$ & $\ldots$ & $\ldots$ & $\ldots$ & $\ldots$ & $\ldots$ & $\ldots$ & $\ldots$ & $0.72 \pm 0.03$ \\
\hline 7320 [O II] & $\ldots$ & $\ldots$ & $\ldots$ & $\ldots$ & $\ldots$ & $\ldots$ & $\ldots$ & $0.49 \pm 0.03$ \\
\hline 7330 [O II] & $\ldots$ & $\ldots$ & $\ldots$ & $\ldots$ & $\ldots$ & $\ldots$ & $\ldots$ & $0.25 \pm 0.03$ \\
\hline$C(\mathrm{H} \beta)^{a}$ & 0.135 & 0.015 & 0.245 & 0.005 & 0.000 & 0.075 & 0.285 & 0.235 \\
\hline$F(\mathrm{H} \beta)^{b}$ & 1.98 & 0.42 & 15.97 & 3.05 & 4.21 & 3.55 & 81.87 & 19.30 \\
\hline $\mathrm{EW}(\mathrm{H} \beta)(\AA)$ & 36.1 & 23.1 & 220.6 & 18.3 & 36.5 & 38.7 & 241.8 & 213.6 \\
\hline \multirow[t]{4}{*}{ EW(abs) $(\AA)$} & 1.15 & 2.00 & 0.80 & 1.25 & 0.65 & 1.30 & 0.65 & 0.60 \\
\hline & \multicolumn{8}{|c|}{ GALAXY } \\
\hline & J2104-0035 & J2104-0035 & J2104-0035 & $\mathrm{J} 2302+0049$ & $\mathrm{~J} 2302+0049$ & $\mathrm{~J} 2324-0006$ & $\mathrm{~J} 2354-0004$ & \\
\hline & No. 2 & No. 3 & No. 4 & No. 1 & No. 2 & & No. 1 & \\
\hline 3727 [O II] & $114.48 \pm 11.06$ & $108.68 \pm 4.99$ & $88.02 \pm 3.86$ & $59.42 \pm 1.00$ & $190.27 \pm 4.15$ & $152.34 \pm 2.43$ & $138.52 \pm 11.46$ & \\
\hline $3750 \mathrm{H} 12$ & $\ldots$ & $\ldots$ & $\ldots$ & $3.18 \pm 0.25$ & $\ldots$ & $3.14 \pm 0.33$ & $\ldots$ & \\
\hline 3771 H11 & $\ldots$ & $\ldots$ & $\ldots$ & $4.04 \pm 0.23$ & $\ldots$ & $3.83 \pm 0.33$ & $\ldots$ & \\
\hline 3798 H10 & $\ldots$ & $\ldots$ & $\ldots$ & $6.34 \pm 0.24$ & $\ldots$ & $6.29 \pm 0.32$ & $\ldots$ & \\
\hline $3820 \mathrm{He}$ I & $\ldots$ & $\ldots$ & $\ldots$ & $1.21 \pm 0.14$ & $\ldots$ & $\ldots$ & $\ldots$ & \\
\hline 3835 H9 & $\ldots$ & $\ldots$ & $\ldots$ & $7.43 \pm 0.24$ & $\ldots$ & $7.53 \pm 0.31$ & $\ldots$ & \\
\hline 3868 [Ne III] & $\ldots$ & $\ldots$ & $\ldots$ & $45.23 \pm 0.72$ & $40.05 \pm 1.31$ & $47.39 \pm 0.75$ & $\ldots$ & \\
\hline $3889 \mathrm{He} \mathrm{I} \mathrm{+} \mathrm{H8}$ & $\ldots$ & $16.40 \pm 1.55$ & $22.24 \pm 1.54$ & $20.52 \pm 0.39$ & $22.92 \pm 1.37$ & $21.00 \pm 0.42$ & $\ldots$ & \\
\hline $3968[\mathrm{Ne}$ III $]+\mathrm{H} 7$ & $\ldots$ & $17.76 \pm 1.60$ & $14.37 \pm 1.37$ & $29.75 \pm 0.50$ & $25.90 \pm 1.29$ & $31.37 \pm 0.54$ & $\ldots$ & \\
\hline $4026 \mathrm{He} \mathrm{I}$ & $\ldots$ & $\ldots$ & $\ldots$ & $1.64 \pm 0.10$ & $\ldots$ & $1.84 \pm 0.11$ & $\ldots$ & \\
\hline 4068 [S II] & $\ldots$ & $\ldots$ & $\ldots$ & $\ldots$ & $\ldots$ & $1.19 \pm 0.09$ & $\ldots$ & \\
\hline 4076 [S II] & $\ldots$ & $\ldots$ & $\ldots$ & $\ldots$ & $\ldots$ & $0.41 \pm 0.08$ & $\ldots$ & \\
\hline $4101 \mathrm{H} \delta$ & $\ldots$ & $24.53 \pm 1.22$ & $24.61 \pm 1.15$ & $26.13 \pm 0.43$ & $30.30 \pm 1.24$ & $26.61 \pm 0.45$ & $25.66 \pm 2.60$ & \\
\hline $4340 \mathrm{H} \gamma$ & $51.10 \pm 3.95$ & $49.03 \pm 1.34$ & $48.00 \pm 1.33$ & $47.28 \pm 0.72$ & $46.12 \pm 1.24$ & $47.16 \pm 0.71$ & $48.20 \pm 2.01$ & \\
\hline 4363 [O III] & $\ldots$ & $3.59 \pm 0.65$ & $1.61 \pm 0.69$ & $14.90 \pm 0.24$ & $6.28 \pm 0.54$ & $9.47 \pm 0.16$ & $4.81 \pm 1.57$ & \\
\hline $4387 \mathrm{He} \mathrm{I}$ & $\ldots$ & $\ldots$ & $\ldots$ & $\ldots$ & $\ldots$ & $0.31 \pm 0.08$ & $\ldots$ & \\
\hline $4471 \mathrm{He} \mathrm{I}$ & $\ldots$ & $\ldots$ & $\ldots$ & $3.55 \pm 0.09$ & $\ldots$ & $3.70 \pm 0.08$ & $\ldots$ & \\
\hline 4658 [Fe III] & $\ldots$ & $\ldots$ & $\ldots$ & $0.49 \pm 0.08$ & $\ldots$ & $0.59 \pm 0.05$ & $\ldots$ & \\
\hline 4686 He II & $\ldots$ & $\ldots$ & $\ldots$ & $2.40 \pm 0.08$ & $\ldots$ & $0.76 \pm 0.05$ & $\ldots$ & \\
\hline $4711[\mathrm{Ar}$ IV $]+\mathrm{He}$ I & $\ldots$ & $\ldots$ & $\ldots$ & $2.41 \pm 0.08$ & $\ldots$ & $1.10 \pm 0.05$ & $\ldots$ & \\
\hline $4740[\mathrm{Ar}$ IV $]$ & $\ldots$ & $\ldots$ & $\ldots$ & $1.41 \pm 0.07$ & $\ldots$ & $0.42 \pm 0.04$ & $\ldots$ & \\
\hline $4861 \mathrm{H} \beta$ & $100.00 \pm 3.67$ & $100.00 \pm 2.01$ & $100.00 \pm 1.95$ & $100.00 \pm 1.45$ & $100.00 \pm 1.93$ & $100.00 \pm 1.44$ & $100.00 \pm 2.60$ & \\
\hline $4921 \mathrm{He} \mathrm{I}$ & $\ldots$ & $\ldots$ & $\ldots$ & $0.89 \pm 0.05$ & $\ldots$ & $1.02 \pm 0.05$ & $\ldots$ & \\
\hline 4959 [O III] & $18.99 \pm 1.81$ & $32.09 \pm 0.90$ & $18.23 \pm 0.62$ & $198.50 \pm 2.86$ & $105.26 \pm 1.96$ & $182.82 \pm 2.62$ & $59.53 \pm 1.82$ & \\
\hline 4988 [Fe III] & $\ldots$ & $\ldots$ & $\ldots$ & $0.75 \pm 0.05$ & $3.20 \pm 0.39$ & $0.87 \pm 0.04$ & $\ldots$ & \\
\hline 5007 [O III] & $59.43 \pm 2.53$ & $94.72 \pm 1.89$ & $60.24 \pm 1.23$ & $567.92 \pm 7.98$ & $315.09 \pm 5.54$ & $543.75 \pm 7.78$ & $166.78 \pm 3.88$ & \\
\hline $5015 \mathrm{He} \mathrm{I}$ & $\ldots$ & $\ldots$ & $\ldots$ & $1.78 \pm 0.06$ & $\ldots$ & $0.96 \pm 0.05$ & $\ldots$ & \\
\hline $5518[\mathrm{Cl} \mathrm{III}]$ & $\ldots$ & $\ldots$ & $\ldots$ & $\ldots$ & $\ldots$ & $0.25 \pm 0.04$ & $\ldots$ & \\
\hline $5538[\mathrm{Cl} \mathrm{III}]$ & $\ldots$ & $\ldots$ & $\ldots$ & $\ldots$ & $\ldots$ & $0.22 \pm 0.03$ & $\ldots$ & \\
\hline $5876 \mathrm{He} \mathrm{I}$ & $5.84 \pm 0.74$ & $9.56 \pm 0.57$ & $9.39 \pm 0.52$ & $10.00 \pm 0.18$ & $8.03 \pm 0.45$ & $10.10 \pm 0.16$ & $9.03 \pm 0.67$ & \\
\hline 6300 [O I] & $\ldots$ & $\ldots$ & $\ldots$ & $1.30 \pm 0.05$ & $\ldots$ & $2.69 \pm 0.05$ & $\ldots$ & \\
\hline 6312 [S III] & $\ldots$ & $\ldots$ & $\ldots$ & $1.21 \pm 0.06$ & $\ldots$ & $1.66 \pm 0.04$ & $\ldots$ & \\
\hline $6363[\mathrm{O} \mathrm{I}]$ & $\ldots$ & $\ldots$ & $\ldots$ & $0.48 \pm 0.04$ & $\ldots$ & $0.97 \pm 0.03$ & $\ldots$ & \\
\hline 6548 [N II $]$ & $\ldots$ & $\ldots$ & $\ldots$ & $\ldots$ & $\ldots$ & $1.46 \pm 0.04$ & $\ldots$ & \\
\hline $6563 \mathrm{H} \alpha$ & $288.62 \pm 8.99$ & $273.23 \pm 5.26$ & $276.97 \pm 5.20$ & $277.79 \pm 4.33$ & $280.05 \pm 5.31$ & $281.56 \pm 4.37$ & $275.84 \pm 6.36$ & \\
\hline 6583 [N II] & $4.92 \pm 0.56$ & $4.28 \pm 0.38$ & $3.93 \pm 0.26$ & $1.70 \pm 0.05$ & $6.61 \pm 0.45$ & $4.94 \pm 0.09$ & $4.85 \pm 0.46$ & \\
\hline $6678 \mathrm{He}$ I & $\ldots$ & $3.66 \pm 0.33$ & $2.53 \pm 0.22$ & $2.18 \pm 0.05$ & $2.49 \pm 0.20$ & $2.91 \pm 0.06$ & $\ldots$ & \\
\hline 6717 [S II] & $12.66 \pm 1.06$ & $8.30 \pm 0.40$ & $8.87 \pm 0.34$ & $4.47 \pm 0.08$ & $16.91 \pm 0.51$ & $11.75 \pm 0.19$ & $12.16 \pm 0.69$ & \\
\hline 6731 [S II] & $10.50 \pm 1.04$ & $4.19 \pm 0.29$ & $5.96 \pm 0.35$ & $3.48 \pm 0.07$ & $11.47 \pm 0.39$ & $8.79 \pm 0.14$ & $6.55 \pm 0.56$ & \\
\hline $7065 \mathrm{He} \mathrm{I}$ & $\ldots$ & $\ldots$ & $\ldots$ & $2.18 \pm 0.05$ & $\ldots$ & $2.43 \pm 0.05$ & $\ldots$ & \\
\hline 7136 [Ar III] & $\ldots$ & $\ldots$ & $\ldots$ & $3.41 \pm 0.07$ & $4.56 \pm 0.09$ & $5.80 \pm 0.10$ & $\ldots$ & \\
\hline $7281 \mathrm{He} \mathrm{I}$ & $\ldots$ & $\ldots$ & $\ldots$ & $0.52 \pm 0.03$ & $\ldots$ & $0.59 \pm 0.02$ & $\ldots$ & \\
\hline 7320 [O II] & $\ldots$ & $\ldots$ & $\ldots$ & $0.65 \pm 0.03$ & $\ldots$ & $2.06 \pm 0.04$ & $\ldots$ & \\
\hline 7330 [O II] & $\ldots$ & $\ldots$ & $\ldots$ & $0.55 \pm 0.03$ & $\ldots$ & $1.67 \pm 0.03$ & $\ldots$ & \\
\hline$C(\mathrm{H} \beta)^{a}$ & 0.015 & 0.225 & 0.205 & 0.260 & 0.195 & 0.250 & 0.080 & \\
\hline$F(\mathrm{H} \beta)^{b}$ & 1.10 & 1.81 & 2.02 & 32.24 & 2.59 & 55.62 & 1.05 & \\
\hline$E W(\mathrm{H} \beta)(\AA)$ & 9.6 & 36.3 & 84.2 & 199.4 & 41.9 & 219.5 & 25.0 & \\
\hline$E W(\mathrm{abs})(\AA)$ & 2.00 & 0.65 & 0.85 & 0.10 & 2.55 & 0.15 & 0.90 & \\
\hline
\end{tabular}

${ }^{a}$ Zero value is assumed if a negative value is derived. ${ }^{b}$ In units of $10^{-16} \mathrm{erg} \mathrm{s}^{-1} \mathrm{~cm}^{-2}$. 
N. G. Guseva et al.: The L-Z relation for a large sample of low-metallicity galaxies, Online Material $p 7$

Table 5. Ionic and total heavy element abundances (3.6 m ESO observations).

\begin{tabular}{|c|c|c|c|c|c|c|c|c|}
\hline \multirow[b]{2}{*}{ Property } & \multicolumn{8}{|c|}{ GALAXY } \\
\hline & J0015+0104 & J0016+0108 & J0029-0108 & J0029-0025 & J0057-0022 & J0107+0001 & J0109+0107 & $\begin{array}{r}\text { J0126-0038 } \\
\text { No. } 1\end{array}$ \\
\hline$T T_{\mathrm{e}}(\mathrm{O}$ III) $(\mathrm{K})$ & $20000 \pm 1020$ & $17023 \pm 1008$ & $18580 \pm 1188$ & $19025 \pm 1020$ & $16258 \pm 215$ & $20000 \pm 1054$ & $12337 \pm 558$ & $14070 \pm 351$ \\
\hline$T_{\mathrm{e}}(\mathrm{O}$ II $)(\mathrm{K})$ & $16284 \pm 1293$ & $15379 \pm 1217$ & $15961 \pm 1470$ & $16083 \pm 1273$ & $15006 \pm 180$ & $16284 \pm 1336$ & $12196 \pm 517$ & $13624 \pm 313$ \\
\hline $\mathrm{O}^{+} / \mathrm{H}^{+}\left(\times 10^{4}\right)$ & $0.084 \pm 0.017$ & $0.186 \pm 0.038$ & $0.129 \pm 0.037$ & $0.096 \pm 0.019$ & $0.199 \pm 0.008$ & $0.115 \pm 0.025$ & $0.381 \pm 0.052$ & $0.294 \pm 0.020$ \\
\hline $\mathrm{O}^{++} / \mathrm{H}^{+}\left(\times 10^{4}\right)$ & $0.033 \pm 0.004$ & $0.153 \pm 0.022$ & $0.096 \pm 0.019$ & $0.099 \pm 0.013$ & $0.200 \pm 0.007$ & $0.053 \pm 0.007$ & $0.571 \pm 0.076$ & $0.534 \pm 0.036$ \\
\hline $\mathrm{O}^{+++} / \mathrm{H}^{+}\left(\times 10^{6}\right)$ & $\ldots$ & $\ldots$ & $\ldots$ & $\ldots$ & $\ldots$ & $\ldots$ & $\ldots$ & $2.421 \pm 0.457$ \\
\hline $\mathrm{O} / \mathrm{H}\left(\times 10^{4}\right)$ & $0.117 \pm 0.017$ & $0.339 \pm 0.044$ & $0.225 \pm 0.042$ & $0.194 \pm 0.023$ & $0.399 \pm 0.011$ & $0.168 \pm 0.026$ & $0.952 \pm 0.092$ & $0.852 \pm 0.042$ \\
\hline $12+\log (\mathrm{O} / \mathrm{H})$ & $7.070 \pm 0.063$ & $7.530 \pm 0.056$ & $7.353 \pm 0.080$ & $7.289 \pm 0.052$ & $7.601 \pm 0.012$ & $7.226 \pm 0.066$ & $7.979 \pm 0.042$ & $7.931 \pm 0.021$ \\
\hline $\mathrm{Ne}^{++} / \mathrm{H}^{+}\left(\times 10^{5}\right)$ & $\ldots$ & $\ldots$ & $\ldots$ & $\ldots$ & $0.660 \pm 0.048$ & $\ldots$ & $1.498 \pm 0.220$ & $1.247 \pm 0.092$ \\
\hline ICF & $\ldots$ & $\ldots$ & $\ldots$ & $\ldots$ & 1.213 & $\ldots$ & 1.155 & 1.141 \\
\hline $\log (\mathrm{Ne} / \mathrm{O})$ & $\ldots$ & $\ldots$ & $\ldots$ & $\ldots$ & $-0.697 \pm 0.053$ & $\ldots$ & $-0.740 \pm 0.101$ & $-0.777 \pm 0.049$ \\
\hline \multirow{3}{*}{ Property } & \multicolumn{8}{|c|}{ GALAXY } \\
\hline & J0126-0038 & J0135-0023 & J0213-0002 & J0213-0002 & J0216+0115 & J0216+0115 & 096632 & $\mathrm{~J} 0252+0017$ \\
\hline & No. 2 & & No. 1 & No. 2 & No. 1 & No. 2 & & \\
\hline$T T_{\mathrm{e}}(\mathrm{O}$ III) $(\mathrm{K})$ & $17255 \pm 1005$ & $18365 \pm 1022$ & $16036 \pm 1017$ & $14366 \pm 1019$ & $16076 \pm 1009$ & $17938 \pm 1023$ & $17425 \pm 1005$ & $15617 \pm 1064$ \\
\hline$T_{\mathrm{e}}(\mathrm{O}$ II $)(\mathrm{K})$ & $15481 \pm 1218$ & $15895 \pm 1260$ & $14888 \pm 1209$ & $13839 \pm 1182$ & $14910 \pm 1200$ & $15750 \pm 1253$ & $15552 \pm 1221$ & $14650 \pm 1256$ \\
\hline $\mathrm{O}^{+} / \mathrm{H}^{+}\left(\times 10^{4}\right)$ & $0.185 \pm 0.037$ & $0.135 \pm 0.028$ & $0.169 \pm 0.037$ & $0.341 \pm 0.084$ & $0.208 \pm 0.045$ & $0.185 \pm 0.038$ & $0.249 \pm 0.049$ & $0.251 \pm 0.063$ \\
\hline $\mathrm{O}^{++} / \mathrm{H}^{+}\left(\times 10^{4}\right)$ & $0.136 \pm 0.019$ & $0.102 \pm 0.014$ & $0.263 \pm 0.043$ & $0.413 \pm 0.080$ & $0.229 \pm 0.036$ & $0.091 \pm 0.013$ & $0.078 \pm 0.011$ & $0.255 \pm 0.048$ \\
\hline $\mathrm{O} / \mathrm{H}\left(\times 10^{4}\right)$ & $0.321 \pm 0.042$ & $0.238 \pm 0.031$ & $0.432 \pm 0.057$ & $0.754 \pm 0.116$ & $0.437 \pm 0.058$ & $0.276 \pm 0.040$ & $0.327 \pm 0.051$ & $0.506 \pm 0.079$ \\
\hline $12+\log (\mathrm{O} / \mathrm{H})$ & $7.506 \pm 0.057$ & $7.376 \pm 0.056$ & $7.636 \pm 0.057$ & $7.877 \pm 0.067$ & $7.641 \pm 0.057$ & $7.441 \pm 0.063$ & $7.515 \pm 0.067$ & $7.704 \pm 0.068$ \\
\hline $\mathrm{Ne}^{++} / \mathrm{H}^{+}\left(\times 10^{5}\right)$ & $\cdots$ & $\cdots$ & $\ldots$ & $\ldots$ & $\ldots$ & ... & $0.148 \pm 0.026$ & $\ldots$ \\
\hline ICF & $\ldots$ & $\ldots$ & $\ldots$ & $\ldots$ & $\ldots$ & $\ldots$ & 1.408 & $\ldots$ \\
\hline $\log (\mathrm{Ne} / \mathrm{O})$ & $\ldots$ & $\ldots$ & $\ldots$ & $\ldots$ & $\ldots$ & $\ldots$ & $-1.195 \pm 0.233$ & $\ldots$ \\
\hline \multirow{3}{*}{ Property } & \multicolumn{8}{|c|}{ GALAXY } \\
\hline & $\mathrm{J} 0256+0036$ & J0303-0109 & J0303-0109 & J0341-0026 & J0341-0026 & J0341-0026 & G1815-6701 & G2052-6912 \\
\hline & & No. 1 & No. 2 & No. 1 & No. 2 & No. 3 & & No. 1 \\
\hline$T_{\mathrm{e}}(\mathrm{O}$ III $)(\mathrm{K})$ & $15446 \pm 1004$ & $15850 \pm 960$ & $20000 \pm 1143$ & $16136 \pm 1023$ & $17233 \pm 1010$ & $19005 \pm 1014$ & $14202 \pm 308$ & $10991 \pm 110$ \\
\hline$T_{\mathrm{e}}(\mathrm{O}$ II $)(\mathrm{K})$ & $14548 \pm 1183$ & $14784 \pm 816$ & $16284 \pm 1449$ & $14942 \pm 1218$ & $15472 \pm 1223$ & $16078 \pm 1264$ & $13721 \pm 273$ & $11007 \pm 105$ \\
\hline $\mathrm{O}^{+} / \mathrm{H}^{+}\left(\times 10^{4}\right)$ & $0.190 \pm 0.042$ & $0.131 \pm 0.020$ & $0.121 \pm 0.030$ & $0.209 \pm 0.046$ & $0.113 \pm 0.023$ & $0.064 \pm 0.013$ & $0.318 \pm 0.019$ & $0.370 \pm 0.014$ \\
\hline $\mathrm{O}^{++} / \mathrm{H}^{+}\left(\times 10^{4}\right)$ & $0.327 \pm 0.055$ & $0.359 \pm 0.055$ & $0.046 \pm 0.008$ & $0.222 \pm 0.036$ & $0.189 \pm 0.027$ & $0.120 \pm 0.015$ & $0.645 \pm 0.038$ & $1.281 \pm 0.043$ \\
\hline $\mathrm{O}^{+++} / \mathrm{H}^{+}\left(\times 10^{6}\right)$ & $\ldots$ & $\ldots$ & $\ldots$ & $\ldots$ & $\ldots$ & $\ldots$ & $1.764 \pm 0.426$ & $0.611 \pm 0.106$ \\
\hline $\mathrm{O} / \mathrm{H}\left(\times 10^{4}\right)$ & $0.517 \pm 0.069$ & $0.490 \pm 0.059$ & $0.166 \pm 0.031$ & $0.430 \pm 0.059$ & $0.302 \pm 0.036$ & $0.184 \pm 0.020$ & $0.981 \pm 0.043$ & $1.657 \pm 0.045$ \\
\hline $12+\log (\mathrm{O} / \mathrm{H})$ & $7.713 \pm 0.058$ & $7.690 \pm 0.052$ & $7.221 \pm 0.082$ & $7.634 \pm 0.059$ & $7.480 \pm 0.051$ & $7.265 \pm 0.047$ & $7.992 \pm 0.019$ & $8.219 \pm 0.012$ \\
\hline $\mathrm{Ne}^{++} / \mathrm{H}^{+}\left(\times 10^{5}\right)$ & $0.780 \pm 0.138$ & $0.981 \pm 0.159$ & $\ldots$ & $\ldots$ & $\ldots$ & $\ldots$ & $1.645 \pm 0.105$ & $2.810 \pm 0.109$ \\
\hline ICF & 1.138 & 1.092 & $\ldots$ & $\ldots$ & $\ldots$ & $\ldots$ & 1.126 & 1.125 \\
\hline \multirow[t]{2}{*}{$\log (\mathrm{Ne} / \mathrm{O})$} & $-0.765 \pm 0.121$ & $-0.660 \pm 0.102$ & $\ldots$ & $\ldots$ & $\ldots$ & $\ldots$ & $-0.724 \pm 0.042$ & $-0.720 \pm 0.023$ \\
\hline & \multicolumn{8}{|c|}{ GALAXY } \\
\hline \multirow[t]{2}{*}{ Property } & G2052-6912 & $\mathrm{J} 2053+0039$ & $\mathrm{~J} 2105+0032$ & $\mathrm{~J} 2105+0032$ & J2112-0016 & J2112-0016 & J2119-0732 & $\mathrm{J} 2120-0058$ \\
\hline & No. 2 & & No. 1 & No. 2 & No. 1 & No. 2 & & \\
\hline$T_{\mathrm{e}}(\mathrm{O}$ III) $(\mathrm{K})$ & $11865 \pm 89$ & $18364 \pm 1039$ & $17397 \pm 1027$ & $17930 \pm 1021$ & $11339 \pm 320$ & $12417 \pm 771$ & $14591 \pm 1003$ & $17281 \pm 908$ \\
\hline$T_{\mathrm{e}}(\mathrm{O}$ II $)(\mathrm{K})$ & $11756 \pm 83$ & $15894 \pm 1282$ & $15541 \pm 1247$ & $15747 \pm 1251$ & $11239 \pm 302$ & $12268 \pm 713$ & $13996 \pm 1167$ & $15492 \pm 736$ \\
\hline $\mathrm{O}^{+} / \mathrm{H}^{+}\left(\times 10^{4}\right)$ & $0.218 \pm 0.006$ & $0.065 \pm 0.014$ & $0.089 \pm 0.019$ & $0.146 \pm 0.030$ & $0.264 \pm 0.025$ & $0.192 \pm 0.036$ & $0.183 \pm 0.043$ & $0.207 \pm 0.025$ \\
\hline $\mathrm{O}^{++} / \mathrm{H}^{+}\left(\times 10^{4}\right)$ & $1.309 \pm 0.033$ & $0.150 \pm 0.021$ & $0.194 \pm 0.029$ & $0.119 \pm 0.017$ & $1.366 \pm 0.120$ & $0.834 \pm 0.151$ & $0.487 \pm 0.088$ & $0.235 \pm 0.030$ \\
\hline $\mathrm{O} / \mathrm{H}\left(\times 10^{4}\right)$ & $1.527 \pm 0.034$ & $0.215 \pm 0.026$ & $0.283 \pm 0.035$ & $0.265 \pm 0.034$ & $1.630 \pm 0.123$ & $1.026 \pm 0.156$ & $0.669 \pm 0.098$ & $0.442 \pm 0.039$ \\
\hline $12+\log (\mathrm{O} / \mathrm{H})$ & $8.184 \pm 0.009$ & $7.332 \pm 0.052$ & $7.451 \pm 0.053$ & $7.423 \pm 0.056$ & $8.212 \pm 0.033$ & $8.011 \pm 0.066$ & $7.826 \pm 0.064$ & $7.646 \pm 0.038$ \\
\hline $\mathrm{Ne}^{++} / \mathrm{H}^{+}\left(\times 10^{5}\right)$ & $2.728 \pm 0.079$ & $\ldots$ & $\ldots$ & $\ldots$ & $2.871 \pm 0.284$ & $1.249 \pm 0.261$ & $1.116 \pm 0.214$ & $0.723 \pm 0.093$ \\
\hline ICF & 1.064 & $\ldots$ & $\ldots$ & $\ldots$ & 1.079 & 1.062 & 1.094 & 1.194 \\
\hline $\log (\mathrm{Ne} / \mathrm{O})$ & $-0.721 \pm 0.017$ & $\ldots$ & $\ldots$ & $\ldots$ & $-0.721 \pm 0.058$ & $-0.888 \pm 0.124$ & $-0.739 \pm 0.123$ & $-0.710 \pm 0.096$ \\
\hline \multirow{3}{*}{ Property } & \multicolumn{8}{|c|}{ GALAXY } \\
\hline & $\mathrm{J} 2150+0033$ & G2155-3946 & J2227-0939 & PHL 293B & J2310-0109 No. 1 & $2310-0109$ No. 2 & & \\
\hline & & & & & No. 1 & No. 2 & & \\
\hline$T T_{\mathrm{e}}(\mathrm{O}$ III) $(\mathrm{K})$ & $16314 \pm 1008$ & $16087 \pm 1008$ & $14707 \pm 1007$ & $17410 \pm 228$ & $15417 \pm 1005$ & $15359 \pm 1006$ & & \\
\hline$T_{\mathrm{e}}(\mathrm{O}$ II $)(\mathrm{K})$ & $15036 \pm 845$ & $14915 \pm 1199$ & $14075 \pm 1173$ & $15546 \pm 184$ & $14531 \pm 1183$ & $14495 \pm 867$ & & \\
\hline $\mathrm{O}^{+} / \mathrm{H}^{+}\left(\times 10^{4}\right)$ & $0.171 \pm 0.026$ & $0.298 \pm 0.064$ & $0.219 \pm 0.051$ & $0.053 \pm 0.002$ & $0.128 \pm 0.028$ & $0.179 \pm 0.029$ & & \\
\hline $\mathrm{O}^{++} / \mathrm{H}^{+}\left(\times 10^{4}\right)$ & $0.230 \pm 0.036$ & $0.160 \pm 0.025$ & $0.434 \pm 0.079$ & $0.360 \pm 0.012$ & $0.379 \pm 0.063$ & $0.348 \pm 0.059$ & & \\
\hline $\mathrm{O}^{+++} / \mathrm{H}^{+}\left(\times 10^{6}\right)$ & $\ldots$ & $\ldots$ & $\ldots$ & $0.655 \pm 0.065$ & $\ldots$ & $\ldots$ & & \\
\hline $\mathrm{O} / \mathrm{H}\left(\times 10^{4}\right)$ & $0.401 \pm 0.044$ & $0.458 \pm 0.068$ & $0.653 \pm 0.094$ & $0.420 \pm 0.012$ & $0.507 \pm 0.069$ & $0.527 \pm 0.066$ & & \\
\hline $12+\log (\mathrm{O} / \mathrm{H})$ & $7.603 \pm 0.048$ & $7.661 \pm 0.065$ & $7.815 \pm 0.062$ & $7.624 \pm 0.013$ & $7.705 \pm 0.060$ & $7.722 \pm 0.054$ & & \\
\hline $\mathrm{Ne}^{++} / \mathrm{H}^{+}\left(\times 10^{5}\right)$ & $\ldots$ & $0.363 \pm 0.070$ & $0.993 \pm 0.202$ & $0.729 \pm 0.026$ & $0.517 \pm 0.094$ & $0.695 \pm 0.127$ & & \\
\hline ICF & $\ldots$ & 1.319 & 1.122 & 1.046 & 1.086 & 1.124 & & \\
\hline $\log (\mathrm{Ne} / \mathrm{O})$ & $\ldots$ & $-0.980 \pm 0.193$ & $-0.768 \pm 0.134$ & $-0.742 \pm 0.021$ & $-0.956 \pm 0.114$ & $-0.829 \pm 0.120$ & & \\
\hline
\end{tabular}


N. G. Guseva et al.: The L-Z relation for a large sample of low-metallicity galaxies, Online Material $p 8$

Table 6. Ionic and total heavy element abundances (VLT observations).

\begin{tabular}{|c|c|c|c|c|c|c|c|c|}
\hline \multirow{3}{*}{ Property } & \multicolumn{8}{|c|}{$\overline{\text { GALAXY }}$} \\
\hline & $\mathrm{J} 0004+0025$ & J0004+0025 & J0014-0044 & J0014-0044 & J0202-0047 & J0301-0059 & J0301-0059 & J0301-0059 \\
\hline & No. 1 & No. 2 & No. 1 & No. 2 & & No. 1 & No. 2 & No. 3 \\
\hline$T_{\mathrm{e}}(\mathrm{O}$ III $)(\mathrm{K})$ & $18630 \pm 1019$ & $16701 \pm 1016$ & $13195 \pm 123$ & $16308 \pm 1006$ & $14607 \pm 277$ & $12582 \pm 585$ & $13999 \pm 1236$ & $16248 \pm 1033$ \\
\hline$T_{\mathrm{e}}(\mathrm{O} \mathrm{II})(\mathrm{K})$ & $15975 \pm 1263$ & $15229 \pm 1221$ & $12939 \pm 112$ & $15033 \pm 1201$ & $14006 \pm 243$ & $12416 \pm 539$ & $13571 \pm 1104$ & $15001 \pm 1232$ \\
\hline$T_{\mathrm{e}}(\mathrm{S}$ III $)(\mathrm{K})$ & $17163 \pm 846$ & $15562 \pm 844$ & $13067 \pm 102$ & $15236 \pm 835$ & $14307 \pm 230$ & $12499 \pm 485$ & $13785 \pm 1026$ & $15186 \pm 857$ \\
\hline$N_{\mathrm{e}}(\mathrm{S} \mathrm{II})\left(\mathrm{cm}^{-3}\right)$ & $193 \pm 128$ & $213 \pm 132$ & $33 \pm 28$ & $167 \pm 78$ & $78 \pm 38$ & $10 \pm 10$ & $10 \pm 10$ & $10 \pm 10$ \\
\hline $\mathrm{O}^{+} / \mathrm{H}^{+}\left(\times 10^{4}\right)$ & $0.164 \pm 0.033$ & $0.224 \pm 0.048$ & $0.146 \pm 0.005$ & $0.274 \pm 0.057$ & $0.218 \pm 0.011$ & $0.565 \pm 0.078$ & $0.381 \pm 0.089$ & $0.300 \pm 0.067$ \\
\hline $\mathrm{O}^{++} / \mathrm{H}^{+}\left(\times 10^{4}\right)$ & $0.073 \pm 0.010$ & $0.156 \pm 0.024$ & $0.960 \pm 0.027$ & $0.158 \pm 0.024$ & $0.580 \pm 0.030$ & $0.485 \pm 0.065$ & $0.353 \pm 0.084$ & $0.141 \pm 0.023$ \\
\hline $\mathrm{O} / \mathrm{H}\left(\times 10^{4}\right)$ & $0.237 \pm 0.034$ & $0.380 \pm 0.053$ & $1.106 \pm 0.028$ & $0.431 \pm 0.062$ & $0.798 \pm 0.032$ & $1.050 \pm 0.101$ & $0.734 \pm 0.123$ & $0.441 \pm 0.071$ \\
\hline $12+\log (\mathrm{O} / \mathrm{H})$ & $7.374 \pm 0.063$ & $7.580 \pm 0.061$ & $8.044 \pm 0.011$ & $7.635 \pm 0.063$ & $7.902 \pm 0.017$ & $8.021 \pm 0.042$ & $7.866 \pm 0.073$ & $7.644 \pm 0.070$ \\
\hline $\mathrm{N}^{+} / \mathrm{H}^{+}\left(\times 10^{6}\right)$ & $1.015 \pm 0.155$ & $0.913 \pm 0.140$ & $0.573 \pm 0.013$ & $1.663 \pm 0.240$ & $0.811 \pm 0.029$ & $2.245 \pm 0.202$ & $2.399 \pm 0.373$ & $2.491 \pm 0.409$ \\
\hline ICF & 1.377 & 1.675 & 7.041 & 1.534 & 3.647 & 1.892 & 1.930 & 1.408 \\
\hline $\log (\mathrm{N} / \mathrm{O})$ & $-1.229 \pm 0.094$ & $-1.396 \pm 0.091$ & $-1.438 \pm 0.015$ & $-1.228 \pm 0.090$ & $-1.431 \pm 0.023$ & $-1.393 \pm 0.057$ & $-1.200 \pm 0.099$ & $-1.099 \pm 0.102$ \\
\hline $\mathrm{Ne}^{++} / \mathrm{H}^{+}\left(\times 10^{5}\right)$ & $\ldots$ & $0.659 \pm 0.138$ & $1.989 \pm 0.064$ & $\ldots$ & $1.196 \pm 0.067$ & $1.118 \pm 0.167$ & $0.764 \pm 0.198$ & $\ldots$ \\
\hline ICF & $\ldots$ & 1.273 & 1.047 & $\ldots$ & 1.094 & 1.268 & 1.226 & $\ldots$ \\
\hline $\log (\mathrm{Ne} / \mathrm{O})$ & $\ldots$ & $-0.656 \pm 0.184$ & $-0.725 \pm 0.019$ & $\ldots$ & $-0.785 \pm 0.035$ & $-0.870 \pm 0.119$ & $-0.894 \pm 0.205$ & $\ldots$ \\
\hline $\mathrm{S}^{+} / \mathrm{H}^{+}\left(\times 10^{6}\right)$ & $0.510 \pm 0.059$ & $0.484 \pm 0.059$ & $0.176 \pm 0.003$ & $0.549 \pm 0.065$ & $0.318 \pm 0.010$ & $0.988 \pm 0.076$ & $0.920 \pm 0.121$ & $1.038 \pm 0.138$ \\
\hline $\mathrm{S}^{++} / \mathrm{H}^{+}\left(\times 10^{6}\right)$ & $\ldots$ & $\ldots$ & $1.393 \pm 0.056$ & $\ldots$ & $1.036 \pm 0.079$ & $0.919 \pm 0.113$ & $\ldots$ & $\ldots$ \\
\hline ICF & $\ldots$ & $\ldots$ & 1.724 & $\ldots$ & 1.142 & 0.897 & $\ldots$ & $\ldots$ \\
\hline $\log (\mathrm{S} / \mathrm{O})$ & $\ldots$ & $\ldots$ & $-1.612 \pm 0.019$ & $\ldots$ & $-1.712 \pm 0.031$ & $-1.788 \pm 0.052$ & $\ldots$ & $\ldots$ \\
\hline $\mathrm{Cl}^{++} / \mathrm{H}^{+}\left(\times 10^{8}\right)$ & $\ldots$ & $\ldots$ & $3.474 \pm 0.410$ & $\ldots$ & $\ldots$ & $\ldots$ & $\ldots$ & $\ldots$ \\
\hline ICF & $\ldots$ & $\ldots$ & 1.243 & $\ldots$ & $\ldots$ & $\ldots$ & $\ldots$ & $\ldots$ \\
\hline $\log (\mathrm{Cl} / \mathrm{O})$ & $\ldots$ & $\ldots$ & $-3.408 \pm 0.052$ & $\ldots$ & $\ldots$ & $\ldots$ & $\ldots$ & $\ldots$ \\
\hline $\mathrm{Ar}^{++} / \mathrm{H}^{+}\left(\times 10^{7}\right)$ & $\ldots$ & $\ldots$ & $3.416 \pm 0.075$ & $\ldots$ & $3.606 \pm 0.116$ & $3.719 \pm 0.336$ & $3.569 \pm 0.440$ & $\ldots$ \\
\hline $\mathrm{Ar}^{+++} / \mathrm{H}^{+}\left(\times 10^{7}\right)$ & $\ldots$ & $\begin{array}{l}\cdots \\
\ldots\end{array}$ & $1.213 \pm 0.103$ & $\begin{array}{l}\cdots \\
\ldots\end{array}$ & $\ldots$ & $\ldots$ & $\begin{array}{c}2.000-0.40 \\
\ldots\end{array}$ & $\begin{array}{l}\cdots \\
\ldots\end{array}$ \\
\hline ICF & $\cdots$ & $\cdots$ & 1.122 & $\cdots$ & 1.141 & 1.097 & 1.043 & $\cdots$ \\
\hline $\log (\mathrm{Ar} / \mathrm{O})$ & $\ldots$ & $\ldots$ & $-2.460 \pm 0.020$ & $\ldots$ & $-2.287 \pm 0.022$ & $-2.410 \pm 0.057$ & $-2.295 \pm 0.090$ & $\ldots$ \\
\hline $\mathrm{Fe}^{++} / \mathrm{H}^{+}\left(\times 10^{6}\right)(4658)$ & $\ldots$ & $\ldots$ & $\ldots$ & $\ldots$ & $\ldots$ & $\ldots$ & $\ldots$ & $\ldots$ \\
\hline $\mathrm{Fe}^{++} / \mathrm{H}^{+}\left(\times 10^{6}\right)(4988)$ & $\ldots$ & $\ldots$ & $0.125 \pm 0.003$ & $\ldots$ & $\ldots$ & $\ldots$ & $\ldots$ & $\ldots$ \\
\hline ICF & $\ldots$ & $\ldots$ & 10.398 & $\ldots$ & $\ldots$ & $\ldots$ & $\ldots$ & $\ldots$ \\
\hline $\log (\mathrm{Fe} / \mathrm{O})(\lambda 4658)$ & $\ldots$ & $\ldots$ & $\ldots$ & $\ldots$ & $\ldots$ & $\ldots$ & $\ldots$ & $\ldots$ \\
\hline $\log (\mathrm{Fe} / \mathrm{O})(4988)$ & $\ldots$ & $\ldots$ & $-1.932 \pm 0.015$ & $\ldots$ & $\ldots$ & $\ldots$ & $\ldots$ & $\ldots$ \\
\hline \multirow{3}{*}{ Property } & \multicolumn{8}{|c|}{ GALAXY } \\
\hline & J0315-0024 & J0315-0024 & $\mathrm{J} 0338+0013$ & G0405-3648 & G0405-3648 & G0405-3648 & J0519+0007 & J2104-0035 \\
\hline & No. 1 & No. 2 & & No. 1 & No. 2 & No. 3 & & No. 1 \\
\hline$T_{\mathrm{e}}(\mathrm{O}$ III $)(\mathrm{K})$ & $12550 \pm 1733$ & $18111 \pm 1012$ & $17882 \pm 208$ & $15013 \pm 1293$ & $18169 \pm 908$ & $21295 \pm 1349$ & $20143 \pm 238$ & $20194 \pm 294$ \\
\hline$T_{\mathrm{e}}(\mathrm{O}$ II $)(\mathrm{K})$ & $12387 \pm 1597$ & $15811 \pm 1243$ & $15729 \pm 165$ & $14278 \pm 1125$ & $15830 \pm 712$ & $16407 \pm 902$ & $16306 \pm 171$ & $16313 \pm 210$ \\
\hline$T_{\mathrm{e}}(\mathrm{S}$ III) $(\mathrm{K})$ & $12468 \pm 1438$ & $16732 \pm 840$ & $16542 \pm 173$ & $14645 \pm 1073$ & $16780 \pm 753$ & $19375 \pm 1119$ & $18419 \pm 198$ & $18461 \pm 244$ \\
\hline$N_{\mathrm{e}}(\mathrm{S} \mathrm{II})\left(\mathrm{cm}^{-3}\right)$ & $1099 \pm 206$ & $10 \pm 10$ & $116 \pm 50$ & $10 \pm 10$ & $108 \pm 57$ & $10 \pm 10$ & $483 \pm 60$ & $84 \pm 74$ \\
\hline $\mathrm{O}^{+} / \mathrm{H}^{+}\left(\times 10^{4}\right)$ & $0.339 \pm 0.138$ & $0.147 \pm 0.030$ & $0.048 \pm 0.002$ & $0.137 \pm 0.030$ & $0.125 \pm 0.014$ & $0.107 \pm 0.014$ & $0.021 \pm 0.001$ & $0.019 \pm 0.001$ \\
\hline $\mathrm{O}^{++} / \mathrm{H}^{+}\left(\times 10^{4}\right)$ & $0.289 \pm 0.115$ & $0.108 \pm 0.014$ & $0.385 \pm 0.012$ & $0.228 \pm 0.051$ & $0.099 \pm 0.012$ & $0.069 \pm 0.010$ & $0.248 \pm 0.007$ & $0.162 \pm 0.006$ \\
\hline $\mathrm{O}^{+++} / \mathrm{H}^{+}\left(\times 10^{6}\right)$ & $\ldots$ & $\ldots$ & $0.531 \pm 0.042$ & $\ldots$ & $\ldots$ & $\ldots$ & $0.444 \pm 0.021$ & $0.126 \pm 0.019$ \\
\hline $\mathrm{O} / \mathrm{H}\left(\times 10^{4}\right)$ & $0.628 \pm 0.179$ & $0.255 \pm 0.033$ & $0.438 \pm 0.012$ & $0.365 \pm 0.059$ & $0.224 \pm 0.018$ & $0.176 \pm 0.017$ & $0.273 \pm 0.007$ & $0.183 \pm 0.006$ \\
\hline $12+\log (\mathrm{O} / \mathrm{H})$ & $7.798 \pm 0.124$ & $7.406 \pm 0.056$ & $7.641 \pm 0.012$ & $7.562 \pm 0.070$ & $7.351 \pm 0.036$ & $7.246 \pm 0.043$ & $7.437 \pm 0.012$ & $7.261 \pm 0.014$ \\
\hline $\mathrm{N}^{+} / \mathrm{H}^{+}\left(\times 10^{6}\right)$ & $0.768 \pm 0.202$ & $0.786 \pm 0.128$ & $0.110 \pm 0.004$ & $0.440 \pm 0.067$ & $0.430 \pm 0.036$ & $0.319 \pm 0.031$ & $0.195 \pm 0.004$ & $0.058 \pm 0.003$ \\
\hline ICF & 1.847 & 1.713 & 8.642 & 2.686 & 1.785 & 1.616 & 12.223 & 8.833 \\
\hline $\log (\mathrm{N} / \mathrm{O})$ & $-1.646 \pm 0.169$ & $-1.277 \pm 0.091$ & $-1.662 \pm 0.019$ & $-1.490 \pm 0.096$ & $-1.465 \pm 0.051$ & $-1.535 \pm 0.061$ & $-1.059 \pm 0.015$ & $-1.551 \pm 0.029$ \\
\hline $\mathrm{Ne}^{++} / \mathrm{H}^{+}\left(\times 10^{5}\right)$ & $0.416 \pm 0.199$ & $\ldots$ & $0.689 \pm 0.022$ & $0.258 \pm 0.071$ & $0.185 \pm 0.028$ & $0.164 \pm 0.024$ & $0.447 \pm 0.013$ & $0.301 \pm 0.011$ \\
\hline ICF & 1.240 & $\ldots$ & 1.040 & 1.143 & 1.251 & 1.286 & 1.031 & 1.037 \\
\hline $\log (\mathrm{Ne} / \mathrm{O})$ & $-1.085 \pm 0.385$ & $\ldots$ & $-0.786 \pm 0.019$ & $-1.094 \pm 0.181$ & $-0.987 \pm 0.123$ & $-0.923 \pm 0.133$ & $-0.774 \pm 0.018$ & $-0.766 \pm 0.022$ \\
\hline $\mathrm{S}^{+} / \mathrm{H}^{+}\left(\times 10^{6}\right)$ & $0.291 \pm 0.066$ & $0.202 \pm 0.028$ & $0.073 \pm 0.002$ & $0.225 \pm 0.028$ & $0.250 \pm 0.016$ & $0.205 \pm 0.016$ & $0.040 \pm 0.001$ & $0.031 \pm 0.001$ \\
\hline $\mathrm{S}^{++} / \mathrm{H}^{+}\left(\times 10^{6}\right)$ & $\ldots$ & $\ldots$ & $0.387 \pm 0.027$ & $\ldots$ & $\ldots$ & $\ldots$ & $0.223 \pm 0.009$ & $0.189 \pm 0.019$ \\
\hline $\mathrm{ICF}$ & $\ldots$ & $\cdots$ & 1.947 & $\cdots$ & $\ldots$ & $\ldots$ & 2.539 & 1.978 \\
\hline $\log (\mathrm{S} / \mathrm{O})$ & $\ldots$ & $\ldots$ & $-1.689 \pm 0.028$ & $\ldots$ & $\ldots$ & $\ldots$ & $-1.612 \pm 0.018$ & $-1.623 \pm 0.040$ \\
\hline $\mathrm{Ar}^{++} / \mathrm{H}^{+}\left(\times 10^{7}\right)$ & $\ldots$ & $\ldots$ & $1.068 \pm 0.028$ & $1.544 \pm 0.201$ & $0.937 \pm 0.080$ & $0.618 \pm 0.052$ & $0.682 \pm 0.014$ & $0.357 \pm 0.015$ \\
\hline $\mathrm{Ar}^{+++} / \mathrm{H}^{+}\left(\times 10^{7}\right)$ & $\ldots$ & $\ldots$ & $0.919 \pm 0.051$ & $\ldots$ & $\ldots$ & $\ldots$ & $0.762 \pm 0.032$ & $0.371 \pm 0.049$ \\
\hline ICF & $\ldots$ & $\ldots$ & 1.867 & 1.058 & 1.048 & 1.060 & 2.455 & 1.898 \\
\hline $\log (\mathrm{Ar} / \mathrm{O})$ & $\ldots$ & $\ldots$ & $-2.342 \pm 0.027$ & $-2.349 \pm 0.090$ & $-2.358 \pm 0.051$ & $-2.430 \pm 0.056$ & $-2.213 \pm 0.025$ & $-2.431 \pm 0.064$ \\
\hline $\mathrm{Fe}^{++} / \mathrm{H}^{+}\left(\times 10^{6}\right)(4658)$ & $\ldots$ & $\ldots$ & $0.083 \pm 0.010$ & $\ldots$ & $\ldots$ & $\ldots$ & $0.076 \pm 0.007$ & $\cdots$ \\
\hline $\mathrm{Fe}^{++} / \mathrm{H}^{+}\left(\times 10^{6}\right)(4988)$ & $\ldots$ & $\ldots$ & $0.091 \pm 0.009$ & $\ldots$ & $0.327 \pm 0.059$ & $\ldots$ & $0.101 \pm 0.007$ & $0.117 \pm 0.021$ \\
\hline $\mathrm{ICF}$ & $\ldots$ & $\ldots$ & 12.634 & $\ldots$ & 2.360 & $\ldots$ & 18.224 & 12.932 \\
\hline $\log (\mathrm{Fe} / \mathrm{O})(\lambda 4658)$ & $\ldots$ & $\ldots$ & $-1.623 \pm 0.054$ & $\ldots$ & $\ldots$ & $\ldots$ & $-1.298 \pm 0.041$ & $\ldots$ \\
\hline
\end{tabular}


N. G. Guseva et al.: The L-Z relation for a large sample of low-metallicity galaxies, Online Material p 9

Table 6. continued.

\begin{tabular}{|c|c|c|c|c|c|c|c|c|}
\hline \multirow{2}{*}{$\begin{array}{l}\log (\mathrm{Fe} / \mathrm{O})(4988) \\
\text { Property }\end{array}$} & \multicolumn{8}{|c|}{ GALAXY } \\
\hline & $\begin{array}{r}\text { J2104-0035 } \\
\text { No. } 2\end{array}$ & $\begin{array}{r}\mathrm{J} 2104-0035 \\
\text { No. } 3\end{array}$ & $\begin{array}{r}\text { J2104-0035 } \\
\text { No. } 4\end{array}$ & $\begin{array}{r}\mathrm{J} 2302+0049 \\
\text { No. } 1\end{array}$ & $\begin{array}{r}\mathrm{J} 2302+0049 \\
\text { No. } 2\end{array}$ & J2324-0006 & $\begin{array}{r}\text { J2354-0004 } \\
\text { No. } 1\end{array}$ & \\
\hline$T_{\mathrm{e}}(\mathrm{O}$ III $)(\mathrm{K})$ & $20000 \pm 1061$ & $21471 \pm 2559$ & $17794 \pm 4091$ & $17281 \pm 179$ & $15203 \pm 616$ & $14313 \pm 128$ & $18120 \pm 3255$ & \\
\hline$T_{\mathrm{e}}(\mathrm{O}$ II $)(\mathrm{K})$ & $16284 \pm 1345$ & $16411 \pm 1693$ & $15697 \pm 3257$ & $15492 \pm 145$ & $14399 \pm 533$ & $13801 \pm 113$ & $15814 \pm 2559$ & \\
\hline$T_{\mathrm{e}}(\mathrm{S}$ III $)(\mathrm{K})$ & $18300 \pm 880$ & $19521 \pm 2124$ & $16469 \pm 3396$ & $16043 \pm 148$ & $14801 \pm 511$ & $14057 \pm 106$ & $16740 \pm 2702$ & \\
\hline$N_{\mathrm{e}}(\mathrm{S} \mathrm{II})\left(\mathrm{cm}^{-3}\right)$ & $255 \pm 248$ & $10 \pm 10$ & $10 \pm 10$ & $143 \pm 44$ & $10 \pm 10$ & $84 \pm 31$ & $10 \pm 10$ & \\
\hline $\mathrm{O}^{+} / \mathrm{H}^{+}\left(\times 10^{4}\right)$ & $0.083 \pm 0.018$ & $0.075 \pm 0.019$ & $0.070 \pm 0.036$ & $0.050 \pm 0.001$ & $0.196 \pm 0.020$ & $0.182 \pm 0.005$ & $0.107 \pm 0.044$ & \\
\hline $\mathrm{O}^{++} / \mathrm{H}^{+}\left(\times 10^{4}\right)$ & $0.034 \pm 0.004$ & $0.047 \pm 0.012$ & $0.043 \pm 0.024$ & $0.450 \pm 0.012$ & $0.335 \pm 0.035$ & $0.675 \pm 0.018$ & $0.120 \pm 0.051$ & \\
\hline $\mathrm{O}^{+++} / \mathrm{H}^{+}\left(\times 10^{6}\right)$ & $\ldots$ & $\ldots$ & $\ldots$ & $1.342 \pm 0.062$ & $\ldots$ & $0.715 \pm 0.051$ & $\ldots$ & \\
\hline $\mathrm{O} / \mathrm{H}\left(\times 10^{4}\right)$ & $0.117 \pm 0.019$ & $0.122 \pm 0.023$ & $0.113 \pm 0.043$ & $0.513 \pm 0.012$ & $0.531 \pm 0.041$ & $0.864 \pm 0.019$ & $0.227 \pm 0.067$ & \\
\hline $12+\log (\mathrm{O} / \mathrm{H})$ & $7.068 \pm 0.070$ & $7.088 \pm 0.080$ & $7.052 \pm 0.166$ & $7.710 \pm 0.010$ & $7.725 \pm 0.033$ & $7.937 \pm 0.009$ & $7.355 \pm 0.128$ & \\
\hline $\mathrm{N}^{+} / \mathrm{H}^{+}\left(\times 10^{6}\right)$ & $0.310 \pm 0.051$ & $0.266 \pm 0.049$ & $0.265 \pm 0.095$ & $0.117 \pm 0.003$ & $0.528 \pm 0.045$ & $0.431 \pm 0.009$ & $0.322 \pm 0.092$ & \\
\hline ICF & 1.323 & 1.592 & 1.587 & 9.717 & 2.732 & 4.662 & 2.133 & \\
\hline $\log (\mathrm{N} / \mathrm{O})$ & $-1.455 \pm 0.103$ & $-1.462 \pm 0.114$ & $-1.428 \pm 0.230$ & $-1.653 \pm 0.017$ & $-1.566 \pm 0.049$ & $-1.633 \pm 0.013$ & $-1.518 \pm 0.177$ & \\
\hline $\mathrm{Ne}^{++} / \mathrm{H}^{+}\left(\times 10^{5}\right)$ & $\ldots$ & $\ldots$ & $\ldots$ & $0.819 \pm 0.024$ & $1.030 \pm 0.115$ & $1.457 \pm 0.043$ & $\ldots$ & \\
\hline ICF & $\ldots$ & $\ldots$ & $\ldots$ & 1.040 & 1.139 & 1.072 & $\ldots$ & \\
\hline $\log (\mathrm{Ne} / \mathrm{O})$ & $\cdots$ & $\cdots$ & $\cdots$ & $-0.780 \pm 0.017$ & $-0.656 \pm 0.075$ & $-0.743 \pm 0.018$ & $\cdots$ & \\
\hline $\mathrm{S}^{+} / \mathrm{H}^{+}\left(\times 10^{6}\right)$ & $0.198 \pm 0.026$ & $0.103 \pm 0.015$ & $0.132 \pm 0.038$ & $0.073 \pm 0.001$ & $0.293 \pm 0.018$ & $0.232 \pm 0.004$ & $0.164 \pm 0.037$ & \\
\hline $\mathrm{S}^{++} / \mathrm{H}^{+}\left(\times 10^{6}\right)$ & $\ldots$ & $\ldots$ & $\ldots$ & $0.508 \pm 0.027$ & $\ldots$ & $1.045 \pm 0.032$ & $\ldots$ & \\
\hline ICF & $\ldots$ & $\ldots$ & $\ldots$ & 2.124 & $\ldots$ & 1.300 & $\ldots$ & \\
\hline $\log (\mathrm{S} / \mathrm{O})$ & $\ldots$ & $\cdots$ & $\ldots$ & $-1.619 \pm 0.023$ & $\ldots$ & $-1.716 \pm 0.014$ & $\cdots$ & \\
\hline $\mathrm{Cl}^{++} / \mathrm{H}^{+}\left(\times 10^{8}\right)$ & $\ldots$ & $\cdots$ & $\cdots$ & $\ldots$ & $\ldots$ & $1.385 \pm 0.150$ & $\ldots$ & \\
\hline ICF & $\ldots$ & $\ldots$ & $\ldots$ & $\ldots$ & $\ldots$ & 1.193 & $\ldots$ & \\
\hline $\log (\mathrm{Cl} / \mathrm{O})$ & $\ldots$ & $\ldots$ & $\ldots$ & $\ldots$ & $\ldots$ & $-3.719 \pm 0.048$ & $\ldots$ & \\
\hline $\mathrm{Ar}^{++} / \mathrm{H}^{+}\left(\times 10^{7}\right)$ & $\ldots$ & $\ldots$ & $\ldots$ & $1.222 \pm 0.027$ & $1.869 \pm 0.095$ & $2.613 \pm 0.053$ & $\ldots$ & \\
\hline $\mathrm{Ar}^{+++} / \mathrm{H}^{+}\left(\times 10^{7}\right)$ & $\ldots$ & $\ldots$ & $\ldots$ & $1.004 \pm 0.053$ & $\ldots$ & $0.487 \pm 0.052$ & $\ldots$ & \\
\hline ICF & $\ldots$ & $\ldots$ & $\ldots$ & 2.041 & 1.061 & 1.265 & $\ldots$ & \\
\hline $\log (\mathrm{Ar} / \mathrm{O})$ & $\ldots$ & $\ldots$ & $\ldots$ & $-2.313 \pm 0.024$ & $-2.428 \pm 0.040$ & $-2.417 \pm 0.015$ & $\ldots$ & \\
\hline $\mathrm{Fe}^{++} / \mathrm{H}^{+}\left(\times 10^{6}\right)(4658)$ & $\ldots$ & $\ldots$ & $\ldots$ & $0.086 \pm 0.014$ & ... & $0.139 \pm 0.012$ & $\ldots$ & \\
\hline $\mathrm{Fe}^{++} / \mathrm{H}^{+}\left(\times 10^{6}\right)(4988)$ & $\ldots$ & $\ldots$ & $\ldots$ & $0.131 \pm 0.010$ & $0.673 \pm 0.096$ & $0.204 \pm 0.010$ & $\ldots$ & \\
\hline ICF & $\ldots$ & $\ldots$ & $\ldots$ & 14.309 & 3.639 & 6.495 & $\ldots$ & \\
\hline $\log (\mathrm{Fe} / \mathrm{O})(\lambda 4658)$ & $\ldots$ & $\ldots$ & $\ldots$ & $-1.618 \pm 0.069$ & $\ldots$ & $-1.982 \pm 0.039$ & $\ldots$ & \\
\hline $\log (\mathrm{Fe} / \mathrm{O})(4988)$ & $\ldots$ & $\ldots$ & $\ldots$ & $-1.437 \pm 0.034$ & $-1.336 \pm 0.071$ & $-1.815 \pm 0.024$ & $\ldots$ & \\
\hline
\end{tabular}


N. G. Guseva et al.: The L-Z relation for a large sample of low-metallicity galaxies, Online Material p 10

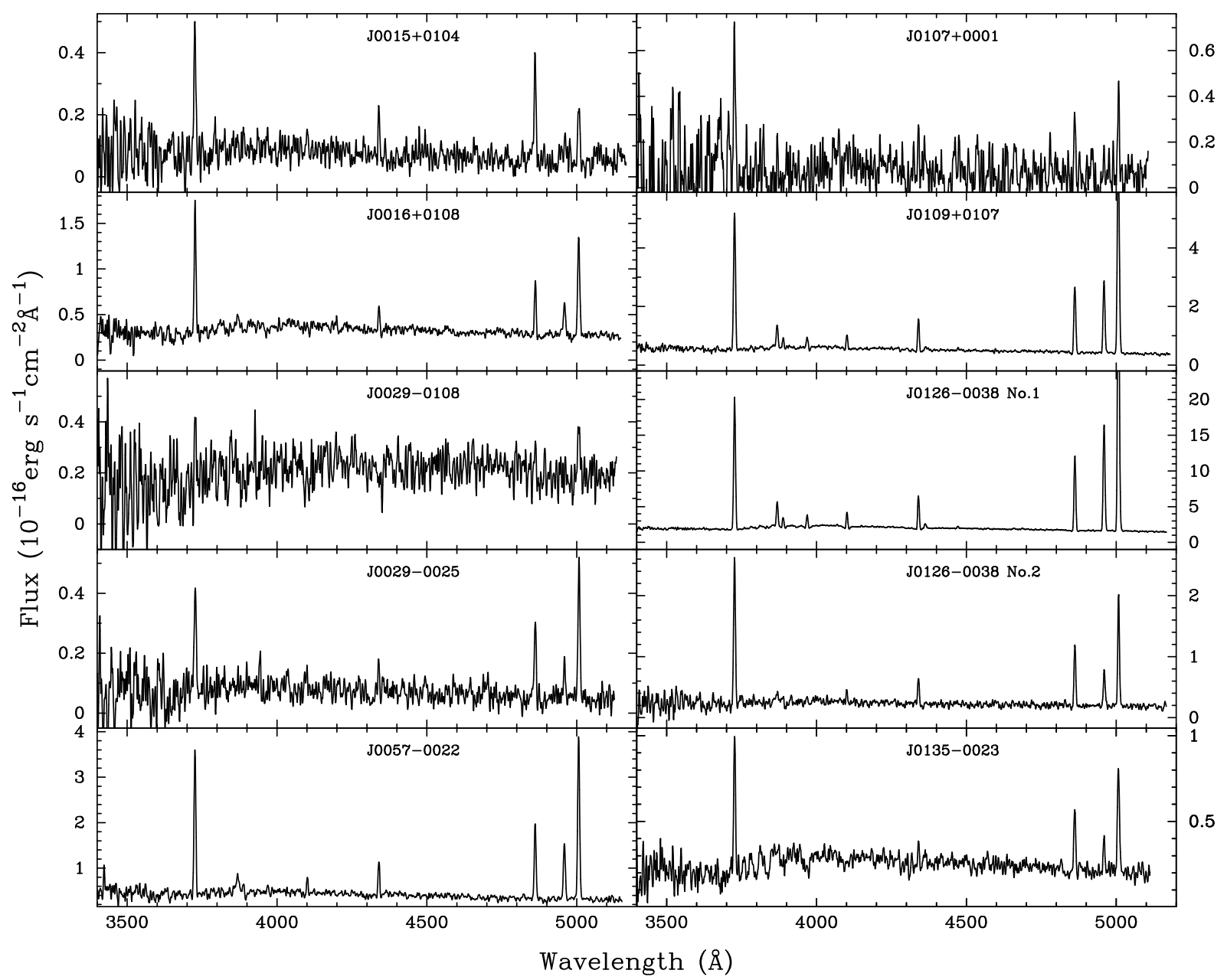

Fig. 1. The flux-calibrated and redshift-corrected $3.6 \mathrm{~m}$ ESO telescope spectra of the emission-line galaxies. 
N. G. Guseva et al.: The L-Z relation for a large sample of low-metallicity galaxies, Online Material p 11

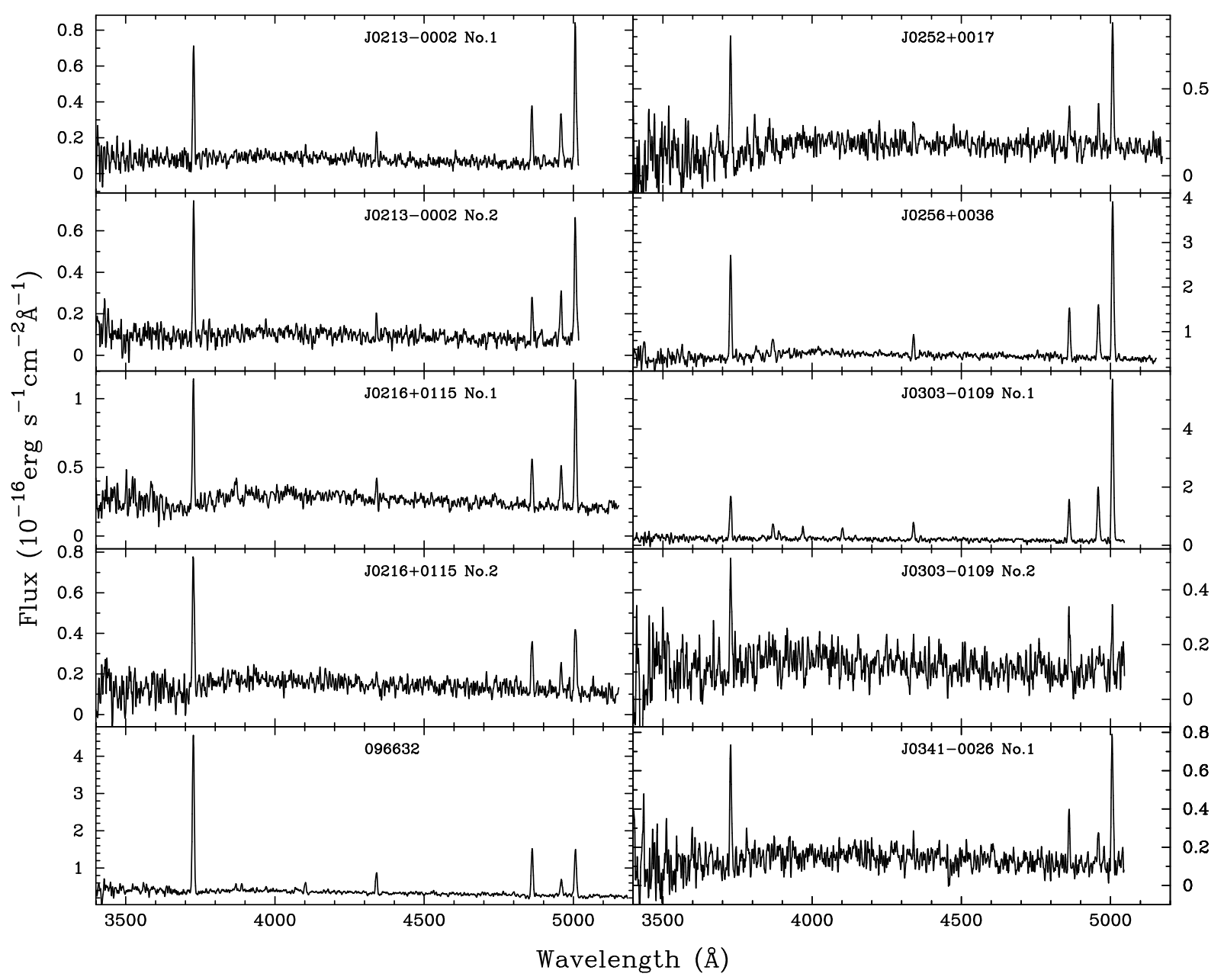

Fig. 1. continued. 
N. G. Guseva et al.: The L-Z relation for a large sample of low-metallicity galaxies, Online Material p 12

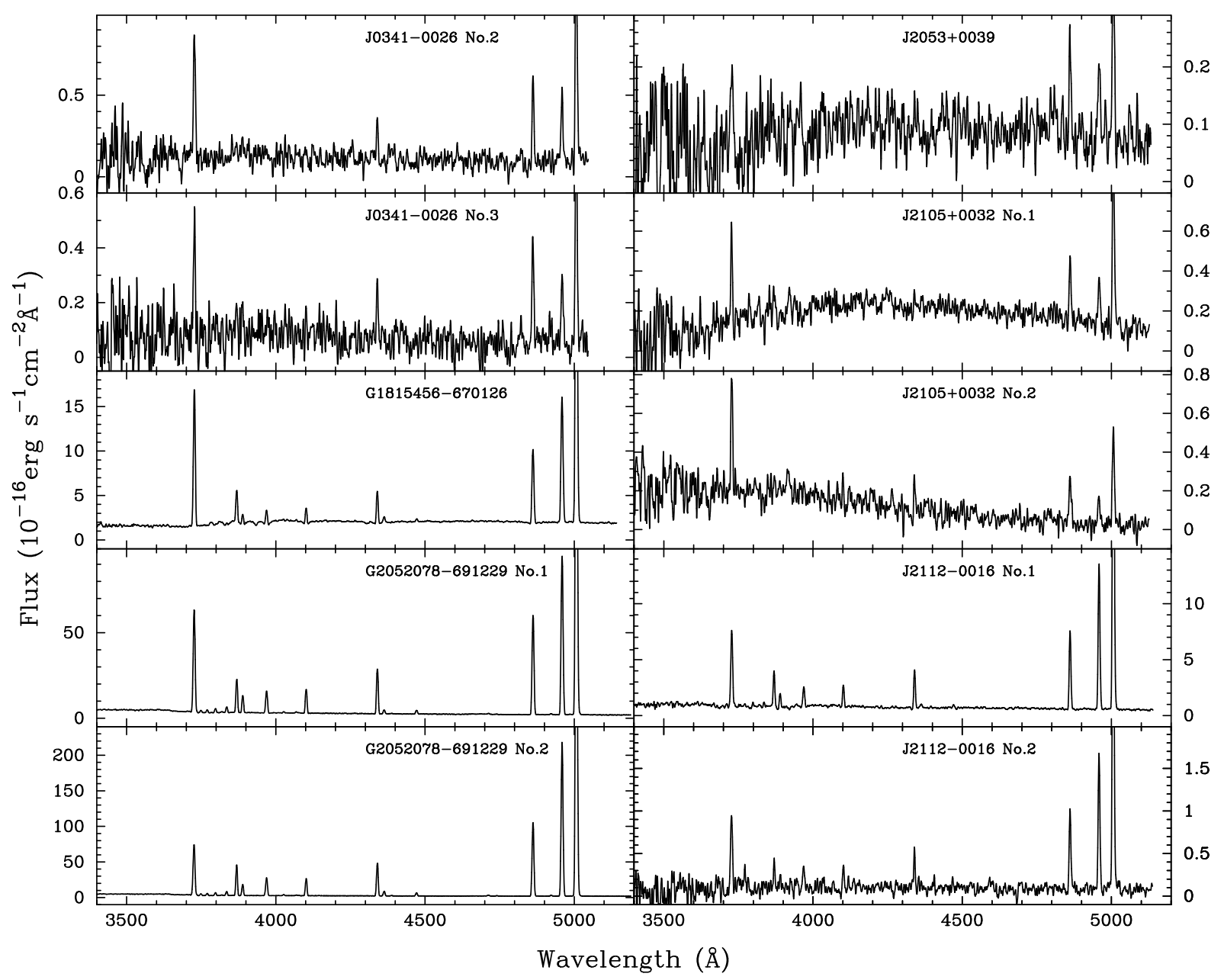

Fig. 1. continued. 
N. G. Guseva et al.: The L-Z relation for a large sample of low-metallicity galaxies, Online Material p 13

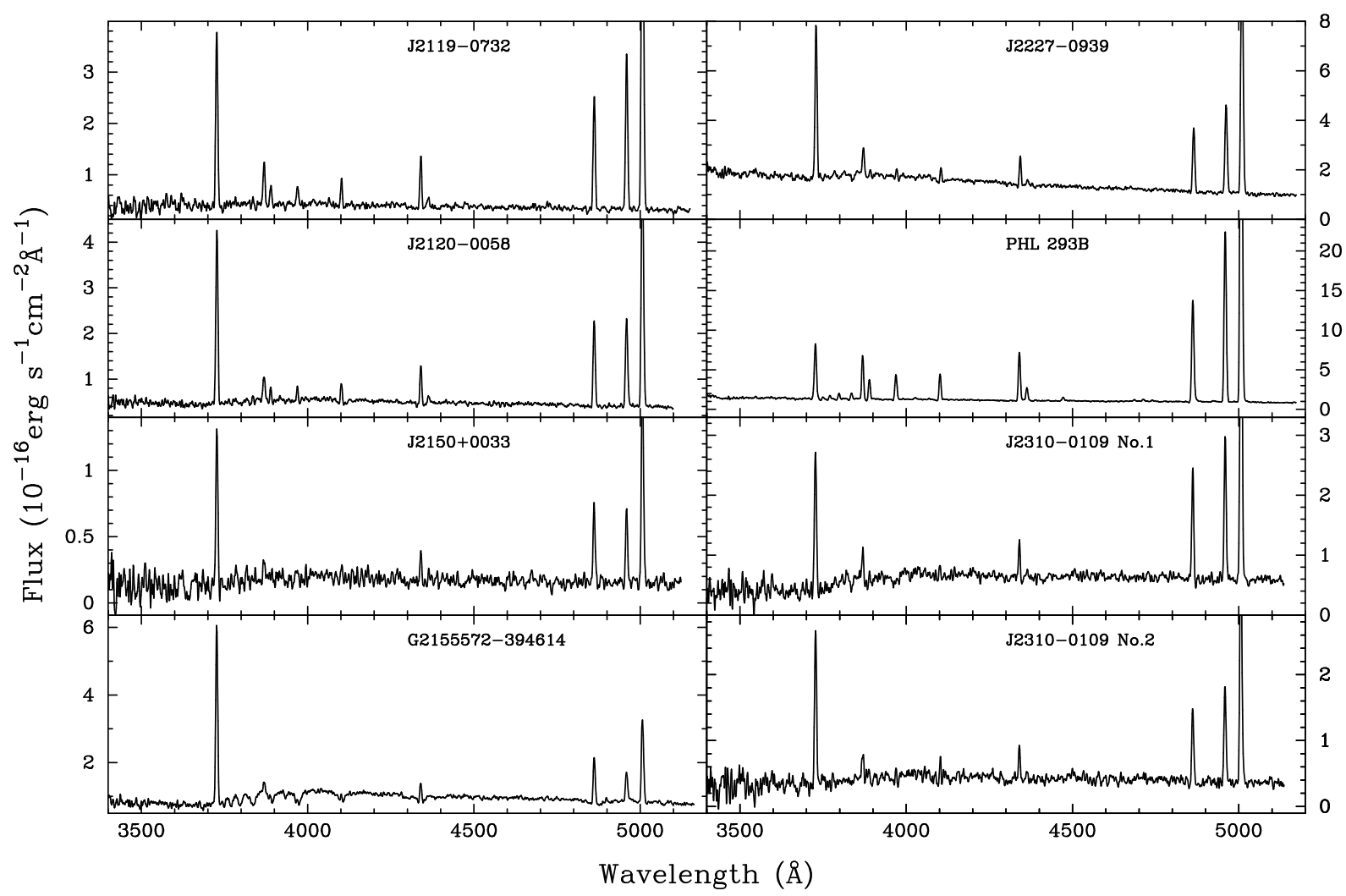

Fig. 1. continued. 
N. G. Guseva et al.: The L-Z relation for a large sample of low-metallicity galaxies, Online Material p 14

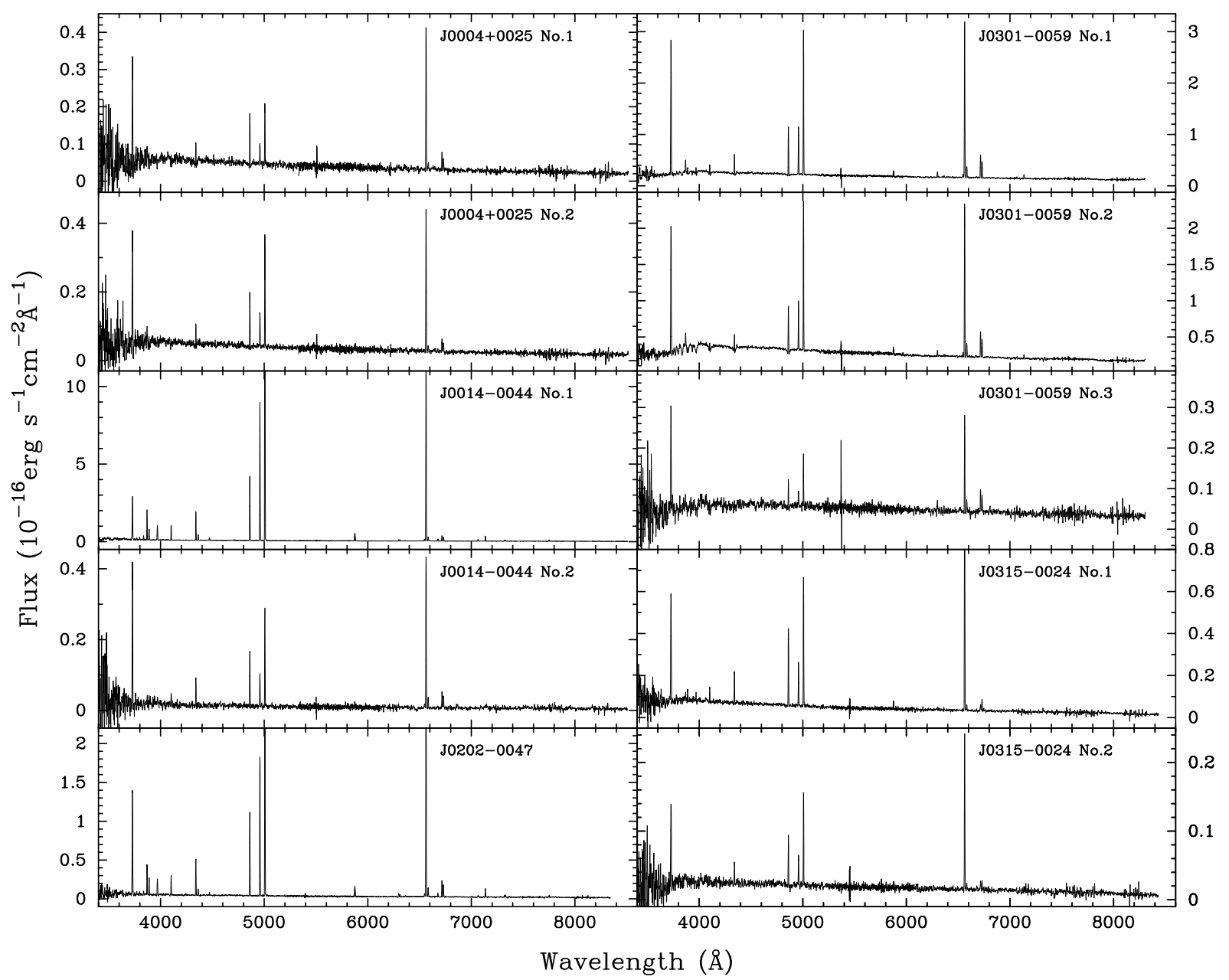

Fig. 2. The flux-calibrated and redshift-corrected VLT spectra of the emission-line galaxies. 
N. G. Guseva et al.: The L-Z relation for a large sample of low-metallicity galaxies, Online Material p 15

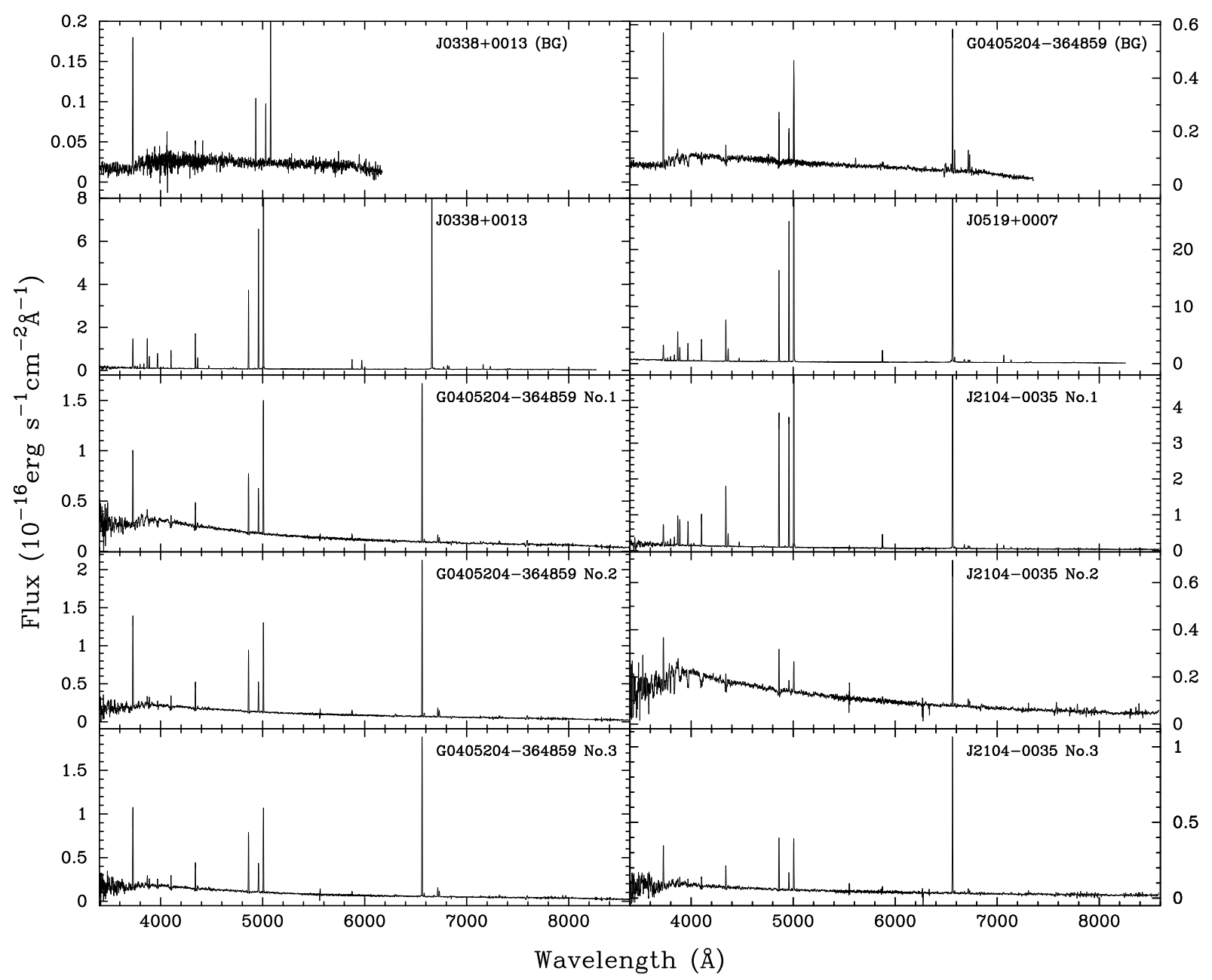

Fig. 2. continued. 
N. G. Guseva et al.: The L-Z relation for a large sample of low-metallicity galaxies, Online Material p 16

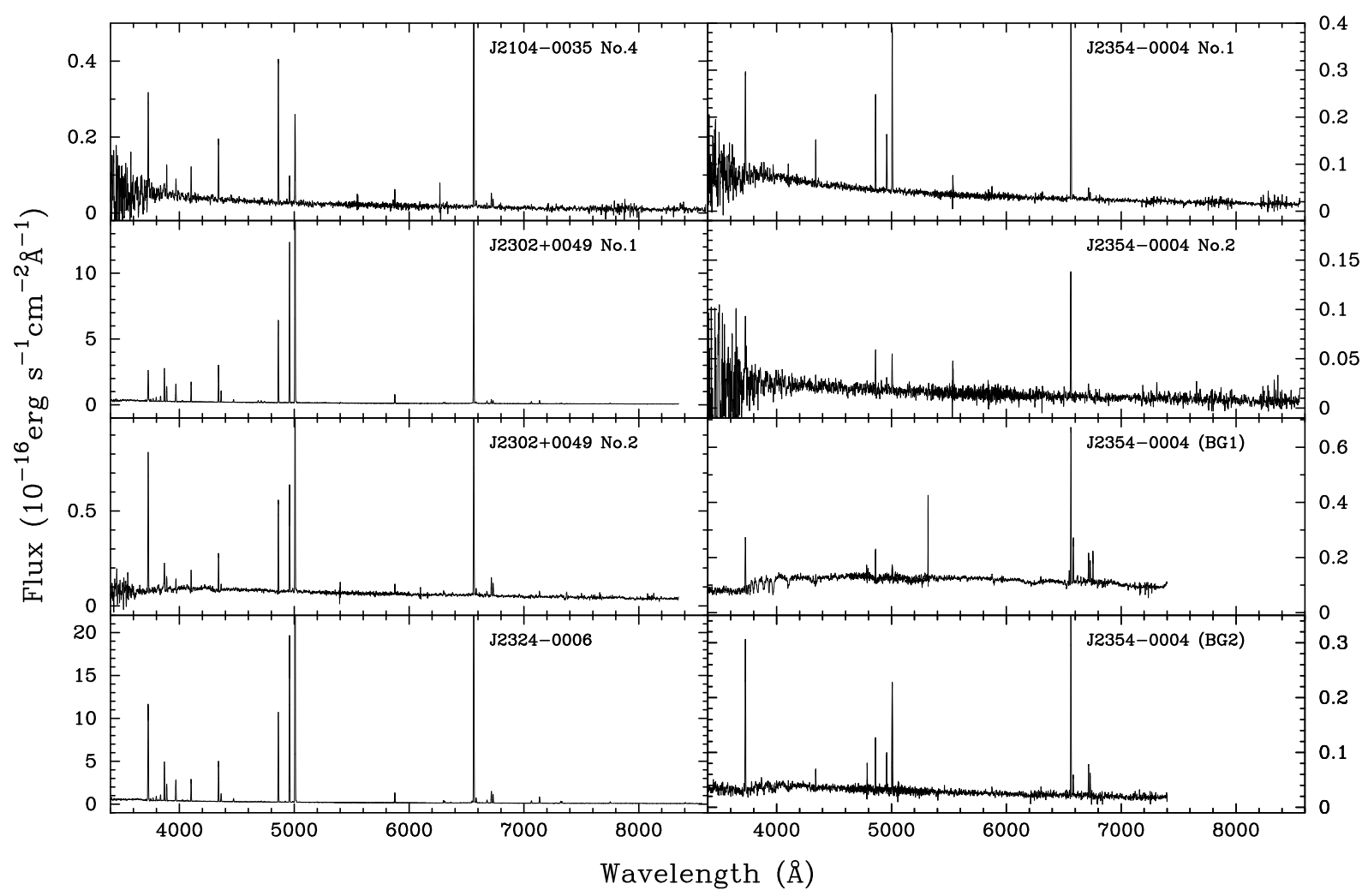

Fig. 2. continued. 